\title{
Transport phenomena in nanofluidics
}

\author{
Reto B. Schoch* \\ Microsystems Laboratory, STI-LMIS, École Polytechnique Fédérale de Lausanne (EPFL), \\ $\mathrm{CH}-1015$ Lausanne, Switzerland; \\ Department of Electrical Engineering and Computer Science, Massachusetts Institute of \\ Technology, 77 Massachusetts Avenue, Cambridge, Massachusetts 02139, USA; \\ and Department of Biological Engineering, Massachusetts Institute of Technology, 77 \\ Massachusetts Avenue, Cambridge, Massachusetts 02139, USA \\ Jongyoon Han \\ Department of Electrical Engineering and Computer Science, Massachusetts Institute of \\ Technology, 77 Massachusetts Avenue, Cambridge, Massachusetts 02139, USA; \\ and Department of Biological Engineering, Massachusetts Institute of Technology, 77 \\ Massachusetts Avenue, Cambridge, Massachusetts 02139, USA \\ Philippe Renaud \\ Microsystems Laboratory, STI-LMIS, École Polytechnique Fédérale de Lausanne (EPFL), \\ CH-1015 Lausanne, Switzerland
}

(Published 17 July 2008)

\begin{abstract}
The transport of fluid in and around nanometer-sized objects with at least one characteristic dimension below $100 \mathrm{~nm}$ enables the occurrence of phenomena that are impossible at bigger length scales. This research field was only recently termed nanofluidics, but it has deep roots in science and technology. Nanofluidics has experienced considerable growth in recent years, as is confirmed by significant scientific and practical achievements. This review focuses on the physical properties and operational mechanisms of the most common structures, such as nanometer-sized openings and nanowires in solution on a chip. Since the surface-to-volume ratio increases with miniaturization, this ratio is high in nanochannels, resulting in surface-charge-governed transport, which allows ion separation and is described by a comprehensive electrokinetic theory. The charge selectivity is most pronounced if the Debye screening length is comparable to the smallest dimension of the nanochannel cross section, leading to a predominantly counterion containing nanometer-sized aperture. These unique properties contribute to the charge-based partitioning of biomolecules at the microchannel-nanochannel interface. Additionally, at this free-energy barrier, size-based partitioning can be achieved when biomolecules and nanoconstrictions have similar dimensions. Furthermore, nanopores and nanowires are rooted in interesting physical concepts, and since these structures demonstrate sensitive, label-free, and real-time electrical detection of biomolecules, the technologies hold great promise for the life sciences. The purpose of this review is to describe physical mechanisms on the nanometer scale where new phenomena occur, in order to exploit these unique properties and realize integrated sample preparation and analysis systems.
\end{abstract}

DOI: $10.1103 /$ RevModPhys.80.839

PACS number(s): 47.61.-k, 82.39.Wj, 73.63.- b, 87.85.Rs

\section{CONTENTS}

I. Introduction

840

II. Electrokinetic Effects

A. Electrostatics in liquids

1. Electrical double layer

2. Potential distribution in EDL

3. Debye-Hückel approximation

4. Gouy-Chapman model

5. Surface charge density
6. Surface conductance

7. Continuum models and molecular-dynamics simulations

B. Electrokinetic effects in nanochannels

1. Basic physics of electrokinetics

2. Comprehensive electrokinetic theory for nanochannels

C. Electrode-electrolyte interface

1. Charge-transfer resistance

2. Constant phase element

III. Phenomena at the Microchannel-Nanochannel Interface

A. Electrical characterization of nanochannels

1. Impedance spectroscopy

2. Nanochannel conductance

3. Ionic current rectification

B. Donnan equilibrium
844

844 whom correspondence should be addressed. reto.schoch@a3.epfl.ch 
1. Donnan potential

2. Membrane potential

C. Exclusion-enrichment effect

1. Permselectivity of a nanochannel

2. Model of the exclusion-enrichment effect

D. Partitioning at the interface

1. Partition coefficient for colloids

2. Electrostatic sieving of proteins

3. Active control of partitioning

E. Concentration polarization

1. Limiting current through nanochannels

2. Electroconvection for mixing

3. Preconcentration of molecules

IV. Macromolecule Separation Mechanisms Using Nanometer-Sized Structures

A. Entropic trapping

B. Ogston sieving

C. Entropic recoil

D. Anisotropy for continuous-flow separation

V. Nanopores and Nanowires for Label-Free Biomolecule Detection

A. Biomolecule translocations through nanopores

1. Biological nanopores

2. Synthetic nanopores

3. Current decrease and increase

B. Nanowire sensors

1. Fabrication techniques

2. Operation mechanism

3. Single-molecule sensitivity

4. Detection efficiency

VI. Fabrication of Nanochannels
A. Wet and dry etching
B. Sacrificial layer methods
C. High-energy beam techniques
D. Nanoimprint lithography
E. Bonding and sealing methods
F. Realization of single nanopores in membranes

VII. Conclusions and Perspectives

Acknowledgments

Lists of Abbreviations and Symbols

References

\section{INTRODUCTION}

Nanofluidics is defined as the study and application of fluid flow in and around nanometer-sized objects with at least one characteristic dimension below $100 \mathrm{~nm}$ (Eijkel and van den Berg, 2005a). At such length scales, structures have a high surface-to-volume ratio, leading to new physical phenomena that are not observed at macrofluidic or microfluidic size scales (Di Ventra et al., 2004; Han, 2004). One significant benefit of nanofluidics is that it presents the possibility of learning new science using controlled regular nanostructures (Mukhopadhyay, 2006), which makes it relevant for many areas in nanoscience and nanotechnology.

The term nanofluidics has only recently been introduced with the rise of micro total analysis systems ( $\mu$ TAS) (Manz et al., 1990), which aim to integrate all steps of biochemical analysis on one microchip (Squires
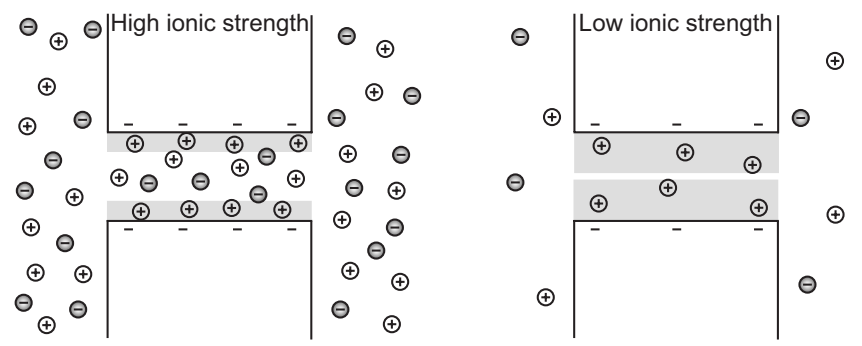

FIG. 1. Electrical double layer (EDL, shaded in gray) at high ionic strength, it is thin, allowing co-ions and counterions to pass through the nanochannel. At low ionic strength, the EDL thickness increases, resulting in a counterion-selective nanochannel. From Schoch, 2006.

and Quake, 2005; Whitesides, 2006). The roots of nanofluidics are broad, and processes on the nanometer scale have implicitly been studied for decades in chemistry, physics, biology, materials science, and many areas of engineering. This dynamic new field has drawn attention in technology, biology, and medicine due to advances in biomolecule preparation and analysis systems (Lieber, 2003), single-molecule interrogations (Craighead, 2003, 2006), and other unique modes of molecular manipulation. For example, molecules can be controlled by charge in nanochannels because of their electrostatic interactions with the electrical double layer (EDL), a shielding layer that is naturally created within the liquid near a charged surface. Moreover, size-based filtration and sieving can be achieved because the length scales of biomolecules and synthetic nanometer-sized objects are similar.

In this review, we present the following unique properties of nanofluidic systems: nanowires can be operated as field-effect transistors to detect chemical and biological species label-free, and transport through nanochannels leads to analyte separation and new phenomena when the EDL thickness becomes comparable to the smallest channel opening (Fig. 1). Such charge-selective features were first described in membrane filtration (using mainly irregular nanoporous systems), and industrial applications have been developed. However, membranes are not in the scope of this review, and the reader is referred to the comprehensive literature of membrane science (Helfferich, 1962; Sata, 2004; Strathmann, 2004). We focus instead on geometrically well-defined, solidstate nanochannels with one cross-sectional dimension between a few nanometers and $100 \mathrm{~nm}$ that are manufactured on a chip with standard microfabrication technology processes. Compared to membranes, which yield statistical results, single, well-designed, and controlled nanochannels are ideal physical modeling systems to study fluidics in a precise manner. Carbon nanotubes are not covered in this review, and the reader is referred to Whitby and Quirke (2007).

The authors are inspired by nature's ultimate nanofluidic system, namely, transmembrane protein channels. They have remarkable features such as high charge selectivity, which was first described in studies of cellfunction-controlling potassium channels on the cell 
membrane. These channels are 10000 times more permeable to potassium than to sodium although both atoms have one positive net charge (Israelachvili, 1992; Doyle et al., 1998). At the same time, these ion channels exhibit high transport throughput, approaching the diffusional limit. Such a performance has not been achieved by any artificial nanopore, which demonstrates that there is much to be learned from biology.

In this review, we first present classic electrostatic and electrokinetic theory relevant to nanofluidics in Sec. II. Expert readers may focus directly on Sec. II.B.2, which describes electrokinetic effects of nanochannels. When a nanochannel is contacted, the geometric size difference between the interfacing channel and the nanochannel leads to a free-energy barrier, and the unique properties of the microchannel-nanochannel interface are analyzed in Sec. III. Section IV focuses on systems that include nanometer-sized structures for separating biomolecules such as DNA and proteins. Then we describe operation mechanisms and physical effects of nanopores and nanowires for label-free biomolecule detection in Sec. V. The fabrication techniques of nanometer-sized openings that we discuss in this review are summarized in Sec. VI. Finally, we draw conclusions and offer perspectives about transport phenomena in nanofluidics.

\section{ELECTROKINETIC EFFECTS}

Here we introduce the reader to electrostatics in liquids and electrokinetic effects, which are the most important and fundamental concepts for the description of transport in nanofluidics. This classical theory was established decades ago in well-established disciplines, but it cannot be omitted in the new interdisciplinary field of nanofluidics (Eijkel and van den Berg, 2005a), and will therefore be summarized.

\section{A. Electrostatics in liquids}

When considering that a solid in contact with a liquid bears a surface charge, one perceives that this parameter is of increased importance in nanochannels, since they have a high surface-to-volume ratio. Surface charge is caused by the dissociation of surface groups and the specific (nonelectric) adsorption of ions in solution to the surface (Perram et al., 1973; Behrens and Grier, 2001). Depending on the number and type of acid and basic groups present in solution (Hunter and Wright, 1971; Davis et al., 1978; Sonnefeld et al., 1995), the solid has either a positive or a negative surface charge density, which is phenomenologically described by $\sigma_{s}=\Sigma_{i} q_{i} / A$, where $q_{i}=z_{i} e$ is the net charge of ion $i, z_{i}$ is the valency of ion $i, e$ is the electron charge, and $A$ is the surface area. A typical value of high charge density and fully ionized surfaces is $\sigma_{s}=0.3 \mathrm{C} \mathrm{m}^{-2}$, which corresponds to one charge per $\sim 0.5 \mathrm{~nm}^{2}$. At a specific $p H$ value of the solution, the surface bears no net charge; this is known as the point of zero charge, which is $\sim 2$ for glass (Parks,
1965; Iler, 1979). We focus on glass for reasons described in Sec. VI to do with the fabrication of nanochannels.

Surface charges result in electrostatic forces, which are important for the description of long-range interactions between molecules and surfaces in liquids, and thus they govern transport in nanofluidic systems. At small distances, van der Waals forces contribute to the attractive part of the interaction, for example, between dissolved particles, whereas coagulation is prevented by repulsive or attractive electrostatic forces. The interplay between van der Waals and electrostatic forces was initially described in the 1940s in the Derjaguin-LandauVerwey-Overbeek (DLVO) theory (Overbeek, 1952; Israelachvili, 1992), which is important for the description of colloidal stability. van der Waals forces are greatly insensitive to variations in electrolyte concentration and $p \mathrm{H}$, which is not true for electrostatic forces.

\section{Electrical double layer}

Due to the fixed surface charge at the solid interface, an oppositely charged region of counterions develops in the liquid to maintain the electroneutrality of the solidliquid interface. This screening region is denoted as the EDL because ideally it consists of opposite charges, some of which are bound while others are mobile.

The electrical double layer was initially represented by a simple capacitor, usually attributed to the model of Helmholtz. Gouy and Chapman treated one layer of charge smeared uniformly over a planar surface immersed in an electrolyte solution (Overbeek, 1952). Stern recognized that the assumptions that the electrolyte ions could be regarded as point charges and the solvent could be treated as a structureless dielectric of constant permittivity were quite unsatisfactory. He introduced the Stern layer between the inner and outer Helmholtz planes, in which the charge and potential distribution are assumed to be linear, and a diffuse layer further from the wall where the Gouy-Chapman theory is applied. This model is presented in Fig. 2, which is separated into three layers (Hunter, 1981). The first layer is at the inner Helmholtz plane and bears the potential $\psi_{i}$, where co-ions and counterions are not hydrated and are specifically adsorbed to the surface. The second layer is defined by the outer Helmholtz plane with potential $\psi_{d}$, consisting of a layer of bound, hydrated, and partially hydrated counterions. The outermost and third layer is the diffuse layer, composed of mobile co-ions and counterions, in which resides the slip plane bearing the $\zeta$ potential (described hereafter). In most cases, the outer Helmholtz plane and the slip plane are situated close to each other (Bhatt et al., 2005), allowing the approximation of $\psi_{d}$ with the $\zeta$ potential for practical purposes.

The slip plane, or shear surface, is an imaginary plane separating ions that are immobile at the surface from those that are mobile in solution. The $\zeta$ potential at this plane can be experimentally determined, and is therefore an important parameter in colloid science for determining the stability of particles, and in $\mu \mathrm{TAS}$ for de- 


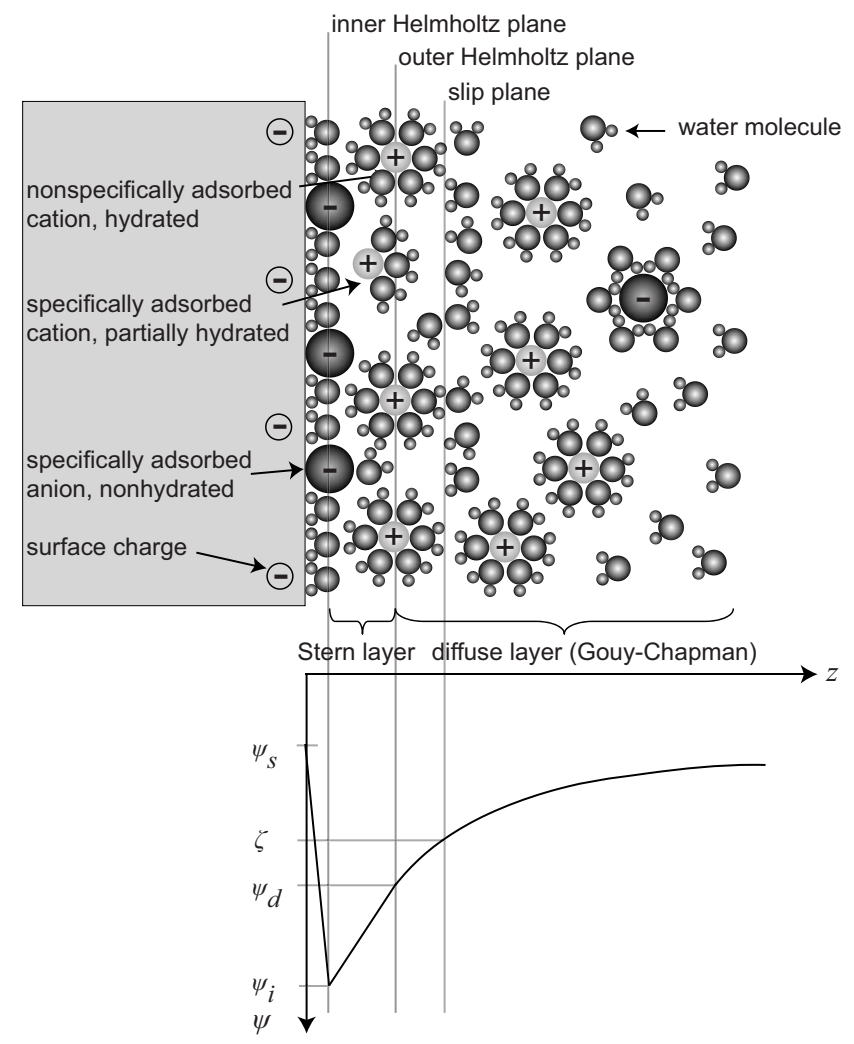

FIG. 2. Gouy-Chapman-Stern model of the solid-electrolyte interface, with the corresponding potential distribution $\psi$ vs the distance $z$ from the wall. The solid is illustrated with a negative surface potential $\psi_{s}$, described by three layers in solution. The inner Helmholtz plane layer $\left(\psi_{i}\right)$ consists of nonhydrated co-ions and counterions, whereas the outer Helmholtz plane layer $\left(\psi_{d}\right)$ is built up of only hydrated counterions. The diffuse layer is defined beyond the outer Helmholtz plane. At the slip plane, the $\zeta$ potential can be experimentally investigated, and as the distance between the outer Helmholtz plane and the slip plane is negligible in most cases, the $\zeta$ potential is usually equal to $\psi_{d}$. Adapted from Schoch et al., 2005.

scribing fluid flow in channels (Dittrich et al., 2006). The $\zeta$ potential is dependent on the $p \mathrm{H}$ and ionic strength of the solution, showing a slight increase in its absolute value for a decreasing number of ions in solution (Lyklema and Overbeek, 1961; Hunter, 1981; Gu and Li, 2000).

\section{Potential distribution in EDL}

We now provide an overview of the potential distribution at charged interfaces; a more comprehensive treatment has been given by Hunter (1981) and Overbeek (1952). To calculate the potential distribution near a charged surface, the electrochemical potential $\tilde{\tilde{\mu}}_{i}$ of ion $i$ in a liquid phase at constant pressure and temperature has to be considered (Israelachvili, 1992),

$$
\tilde{\mu}_{i}=\tilde{\mu}_{i}+z_{i} F \psi=\tilde{\mu}_{i}^{0}+R T \ln \left(\gamma_{a} c_{i} / c^{0}\right)+z_{i} F \psi,
$$

where $\tilde{\mu}_{i}$ is the chemical potential, $F$ is the Faraday constant, $\psi$ is the electric potential due to the surface charge, $\tilde{\mu}_{i}^{0}$ is the standard chemical potential of ion $i$ at constant pressure and temperature, $R$ is the gas constant, $T$ is the temperature, $\gamma_{a}$ is the activity coefficient, $c_{i}$ is the molar concentration of ion $i$, and $c^{0}$ is the standard molarity of $1 \mathrm{~mol} \mathrm{l}^{-1}$. At equilibrium, the electrochemical potential of the ions must be the same everywhere [i.e., $\left.\operatorname{grad}\left(\tilde{\tilde{\mu}}_{i}\right)=0\right]$, and the electrical and diffusional forces on the ion $i$ must be balanced,

$$
\nabla \tilde{\mu}_{i}=-z_{i} F \nabla \psi,
$$

where $\nabla=\operatorname{grad}$. Insertion of the chemical potential $\tilde{\mu}_{i}$ $=\tilde{\mu}_{i}^{0}+R T \ln \left(\gamma_{a} c_{i} / c^{0}\right)$ into Eq. (2), and its integration from a point in the bulk solution where $\psi=0$ and $n_{i}=n_{i}^{\infty}$ and the bulk volume density $n_{i}^{\infty}=1000 N_{A} c_{i}$, leads to the Boltzmann equation, giving the local concentration of each type of ion in the diffuse layer,

$$
n_{i}=n_{i}^{\infty} \exp \left(-z_{i} e \psi / k_{B} T\right),
$$

with $k_{B}$ the Boltzmann constant and the conversion $e / k_{B}=F / R$ is applied. The volume charge density $\rho$ of all ions present in the neighborhood of the surface is given by

$$
\rho=e \sum_{i} n_{i} z_{i} .
$$

One further important equation is required, the fundamental Poisson equation, giving the net excess charge density at a specific distance from the surface:

$$
\nabla^{2} \psi=\frac{d^{2} \psi}{d z^{2}}=-\frac{\rho}{\varepsilon_{0} \varepsilon_{r}},
$$

where $\nabla^{2} \psi=\operatorname{div}(\operatorname{grad} \psi)$ and $z$ is the surface normal direction. Substituting Eqs. (3) and (4) into Eq. (5), we obtain the complete Poisson-Boltzmann equation, which describes how the electrostatic potential due to a distribution of charged atoms varies in space,

$$
\nabla^{2} \psi=\frac{d^{2} \psi}{d z^{2}}=-\frac{e}{\varepsilon_{0} \varepsilon_{r}} \sum_{i} n_{i}^{\infty} z_{i} \exp \left[-z_{i} e \psi(z) / k_{B} T\right] .
$$

\section{Debye-Hückel approximation}

The Poisson-Boltzmann equation [Eq. (6)] is a second-order elliptic partial differential equation, and can be solved analytically by assuming that the surface potential is small everywhere in the EDL $\left(z_{i} \psi_{i}\right.$ $<25.7 \mathrm{mV}$ at $25^{\circ} \mathrm{C}$ ) and by expanding the exponential (using the relation $e^{-\alpha}=1-\alpha$ for small $\alpha$ ), which leads to the Debye-Hückel approximation

$$
\nabla^{2} \psi=\frac{d^{2} \psi}{d z^{2}}=\kappa^{2} \psi(z)
$$

where

$$
\kappa=\left(\frac{e^{2} \sum_{i} n_{i}^{\infty} z_{i}^{2}}{\varepsilon_{0} \varepsilon_{r} k_{B} T}\right)^{1 / 2} .
$$

$\kappa$ is called the Debye-Hückel parameter and is mainly dependent on the bulk volume density $n_{i}^{\infty}$. The potential 
TABLE I. EDL thicknesses $\lambda_{D}$ for typical $\mathrm{KCl}$ concentrations at $25{ }^{\circ} \mathrm{C}$.

\begin{tabular}{lc}
\hline \hline $\mathrm{KCl}$ concentration $(M)$ & Debye length $\lambda_{D}(\mathrm{~nm})$ \\
\hline $10^{0}$ & 0.3 \\
$10^{-1}$ & 1.0 \\
$10^{-2}$ & 3.1 \\
$10^{-3}$ & 9.6 \\
$10^{-4}$ & 30.5 \\
$10^{-5}$ & 96.3 \\
\hline \hline
\end{tabular}

decays exponentially in the diffuse layer with the characteristic distance given by the Debye length $\lambda_{D}=\kappa^{-1}$. This value corresponds to the thickness of the EDL, which increases with dilution as presented in Table I. For a symmetrical $z_{i}: z_{i}$ electrolyte with concentration $c_{i}$ at $25^{\circ} \mathrm{C}$, the value of the Debye length $\lambda_{D}$ (unit is meters) can be written as

$$
\lambda_{D}=\frac{3.04 \times 10^{-10}}{z_{i} \sqrt{c_{i}}}=\frac{2.15 \times 10^{-10}}{\sqrt{I_{s}}},
$$

where the ionic strength $I_{S}$ is

$$
I_{s}=\frac{1}{2} \sum c_{i} z_{i}^{2}
$$

Consider that the region of varying potential extends to a distance of about $3 \lambda_{D}$ before the potential has decayed to about $2 \%$ of its value at the surface. Based on the approximation $z_{i} \psi_{s}<25.7 \mathrm{mV}$, the solution of the Debye-Hückel approximation is

$$
\psi(z)=\psi_{s} \exp (-\kappa z)
$$

\section{Gouy-Chapman model}

As the Debye-Hückel approximation is not valid for high surface potentials, the Poisson-Boltzmann equation has to be solved explicitly. Analytically, this can be done only under the assumption of a symmetrical electrolyte where the valence of the co-ion is equal to the valence of the counterion, leading to the Gouy-Chapman equation

$$
\tanh \left[z_{i} \tilde{\psi}(z) / 4\right]=\tanh \left(z_{i} \tilde{\psi}_{s} / 4\right) \exp (-\kappa z),
$$

where $\tilde{\psi}=e \psi / k_{B} T$ is the dimensionless potential, and at $25^{\circ} \mathrm{C} \tilde{\psi}=1$ for $\psi=25.7 \mathrm{mV}$. For small values of $\alpha$, $\tanh \alpha \approx \alpha$ is valid (Taylor expansion), and Eq. (12) reduces to Eq. (11). The Poisson-Boltzmann equation for high surface potentials and unsymmetrical electrolytes can only be solved numerically.

The resulting ion distribution from the GouyChapman model is presented in Fig. 3(a), where almost all the charge is balanced by the accumulation of counterions and relatively little by the reduction of co-ions. This is why it is possible to treat an unsymmetrical electrolyte system as symmetric, as is done in the GouyChapman model, without incurring too much error. (a)

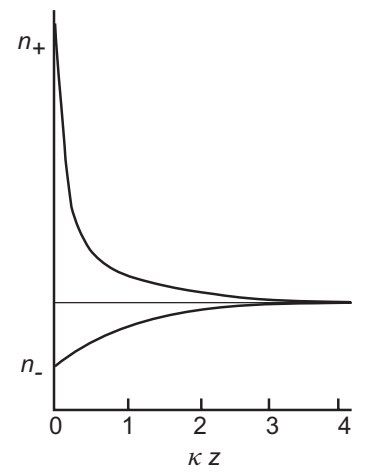

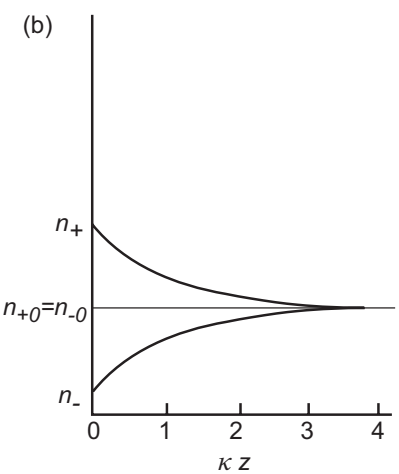

FIG. 3. Volume densities $n_{+}$and $n_{-}$of positive and negative ions, respectively, near a negatively charged surface as a function of the dimensionless number $\kappa z$. Distribution from (a) the Gouy-Chapman model, which shows an excess of positively charged ions, and (b) the Debye-Hückel approximation with a symmetrical co-ion and counterion distribution. Adapted from Hunter, 1981.

When the Debye-Hückel approximation is used instead, the two ion types are of equal significance, as shown in Fig. 3(b).

\section{Surface charge density}

The surface charge density must balance the charge density in the adjacent solution,

$$
\sigma_{s}=-\int_{0}^{\infty} \rho d z
$$

On substituting Eq. (5) and the integrated Eq. (6) into Eq. (13), we obtain a relationship between the surface charge density $\sigma_{s}$ and the surface potential $\psi_{s}$,

$$
\begin{aligned}
\sigma_{s} & =\varepsilon_{0} \varepsilon_{r} \int_{0}^{\infty} \frac{d^{2} \psi}{d z^{2}} d z \\
& =\left(2 \varepsilon_{0} \varepsilon_{r} k_{B} T \sum_{i} n_{i}^{\infty}\left[\exp \left(-z_{i} e \psi_{s} / k_{B} T\right)-1\right]\right)^{1 / 2} d z .
\end{aligned}
$$

Since the surface potential $\psi_{s}$ is difficult to determine experimentally, the charge density of the diffuse layer can be calculated by stopping the integration at the shear plane. We thus achieve an equation that relates the $\zeta$ potential to the diffuse layer charge density,

$$
\sigma_{d}=\left(2 \varepsilon_{0} \varepsilon_{r} k_{B} T \sum_{i} n_{i}^{\infty}\left[\exp \left(-z_{i} e \zeta / k_{B} T\right)-1\right]\right)^{1 / 2} d z,
$$

which has been used, for example, in a site-binding model of the oxide-electrolyte interface (Yates et al., 1974), and has shown consistency with experimental data obtained for oxides by potentiometric titration measurements (Abendrot, 1970). 


\section{Surface conductance}

The EDL contains more ions than the bulk, which results in a surface conductance due to their motion in the electric field (Urban et al., 1934; Kittaka and Morimoto, 1976), and this conductance can be measured in an ac field at sufficiently high frequency. Such conductance measurements can also be performed in a dc field, but the electro-osmotic contributions (see Sec. II.B.1) must be considered. The measured surface conductance $G_{\mathrm{ms}}$ in glass capillaries is given by

$$
G_{\mathrm{ms}}=K^{\sigma} \frac{2 \pi r_{0}}{d}
$$

where $r_{0}$ is the radius of the capillary, $d$ is its length, and $K^{\sigma}$ is the normalized surface conductance, often referred to as the surface conductance in the literature. Decades ago, only the diffuse part of the EDL was considered for the surface conductance $K^{\sigma}$, and values on the order of $10^{-9}-10^{-8} \mathrm{~S}$ were reported (Overbeek, 1952).

However, for nonpenetrable surfaces, Lyklema and Minor (1998) showed that surface conductance does not take place only in the diffuse part of the EDL; a significant amount occurs behind the slip plane, as has been confirmed by their experimental case study of sulfated polystyrene latex plugs. Therefore, they described the total conductance $K^{\sigma}$ as the sum of the surface conductance contributions from the diffuse part $K^{\sigma_{d}}$ and inner part $K^{\sigma_{i}}$ of the EDL (Lyklema, 1995),

$$
K^{\sigma}=K^{\sigma_{d}}+K^{\sigma_{i}}
$$

Lyklema et al. (1998) compared the EDL with a twodimensional gel corresponding to the Stern layer. In this gel, ions can move laterally almost unimpeded, and it is separated from the mobile part of the fluid by the slip plane. For oxides, this hydrodynamically stagnant layer contains up to $85 \%$ of the counterions and therefore contributes significantly to the overall conductance $K^{\sigma}$. Furthermore, the counterion mobility in this stagnant layer is important in silica, for example, with $96 \%$ of the bulk mobility (Lyklema, 2001). In order to relate the surface conductance to the bulk conductivity $K^{L}$, Lyklema and Minor (1998) have introduced the Dukhin number,

$$
\mathrm{Du}=K^{\sigma} / a_{p} K^{L},
$$

where $a_{p}$ is the radius of the particle in solution. If $\mathrm{Du}$ $\gg 1$, the conductance mainly occurs along the charged surface of the particle. Lobbus et al. (2000) have reported that the contribution of the stagnant layer $K^{\sigma_{i}}$ to the total surface conductance $K^{\sigma}$ cannot be neglected, and that the Dukhin number increases with dilution.

\section{Continuum models and molecular-dynamics simulations}

In this review, we focus on nanochannels with heights between a few nanometers and $100 \mathrm{~nm}$, a scale at which continuum and mean-field theories are valid. However, these theories break down in smaller openings because of the collisions between molecules and walls, and the impurities and graininess of the substrate (Fulinski et al., 2004).

At such length scales, molecular-dynamics simulations are used to determine transport in nanopores for a specific period of time with known physical laws (Qiao and Aluru, 2003, 2005; Lu et al., 2004). In a typical molecular-dynamics simulation, a set of molecules with initial random positions is assumed, to which are assigned initial random velocities corresponding to the Boltzmann distribution at the temperature of interest. The time evolution of the atomic positions is obtained by numerically integrating Newton's law of motion for single atoms.

It is important to consider that the applicability of the Gouy-Chapman model and the Poisson-Boltzmann equation are limited by the finite size of the component ions and the effect of these ions on the relative permittivity of the solvent, because of which stochastic, nondeterministic Monte Carlo simulations have been applied (Yang and Yiacoumi, 2002; Fawcett and Smagala, 2006).

It can be difficult to obtain the continuum limit from previously described simulations because simulated systems are usually limited to tens of nanometers and time scales of nanoseconds. This restraint can be overcome with sufficiently coarse-grained simulation techniques such as dissipative particle dynamics (Groot and Warren, 1997) to reveal the physics of nanopores and nanochannels (Duong-Hong et al., 2007). In order to reduce computing time, the density-functional theory has been applied to nanochannels, demonstrating good agreement compared to Monte Carlo and molecular-dynamics simulations (Nilson and Griffiths, 2006).

\section{B. Electrokinetic effects in nanochannels}

Fluid flow in nanochannels is conveniently achieved by electrokinetic techniques or capillary forces. The nonmechanical manipulation of fluid by electrokinetic effects is commonly used and is presented subsequently, whereas capillary forces have been described in the review of microfluidics by Squires and Quake (2005), which we briefly complement with nanofluidic studies.

In nanochannels, capillary pressures up to -17 bars have been reported (Tas et al., 2003), allowing for the spontaneous filling of such channels with liquid. The position of the moving meniscus in nanochannels as a function of time has been modeled (Washburn, 1921; Tas et al., 2004), but after a certain filling length, the formation of bubbles by the enclosure of air is typically encountered, a problem that has been addressed (Han, Mondin, Hegelbach, et al., 2006; Riehn and Austin, 2006). For capillary filling studies, the behavior of liquid in shallow nanochannels down to $6 \mathrm{~nm}$ can be monitored with a Fabry-Perot interferometer by measuring the refractive index of the medium inside the channel (Delft et al., 2007).

Pressure-driven flow can also be used in nanochannels, but high pressures (Tegenfeldt, Prinz, and Cao, 2004) are needed to obtain fluid flow because the pres- 
sure has a power-law relation with the height of the channel (Squires and Quake, 2005). In microchannels, fluid manipulation with hydrostatic pressure is often used, but the achievement of well-controlled fluid flow is challenging (Futterer et al., 2004; Johann and Renaud, 2004; Eijkel and van den Berg, 2005b).

\section{Basic physics of electrokinetics}

The motion of electrically charged molecules and particles due to an applied electric field in moving substances such as water is studied in electrokinetics. This field covers electro-osmosis, electrophoresis, dielectrophoresis, and electrorotation; here we emphasize the first two processes and subsequently present their basic physics.

(a) Electro-osmosis. If a charged surface is in contact with a liquid and an electric field is applied parallel to the interface, movement of liquid adjacent to the wall occurs. If the surface is negatively charged, the net excess of positive ions in the EDL will draw the liquid along because of viscous interactions, which results in flow toward the cathode (Schmid, 1998). At the slip plane, the liquid velocity is zero and increases to a maximum value with the electro-osmotic velocity $v_{\mathrm{eo}}$ at some distance from the wall, after which it remains constant at high ionic strength of the solution in a nanochannel. This plug flow was first described by Smoluchowski (1916, 1918),

$$
v_{\mathrm{eo}}=-\frac{\varepsilon_{0} \varepsilon_{r} \zeta E_{x}}{\eta}
$$

where $E_{x}$ is the electric field in the $x$ direction and $\eta$ is the dynamic (shear) viscosity of the fluid. The electroosmotic mobility is $\mu_{\mathrm{eo}}=\varepsilon_{0} \varepsilon_{r} \zeta / \eta$. The $\zeta$ potential can be changed by active control of electro-osmotic flow using light (Moorthy et al., 2001), the inversion of electroosmotic flow with surface coatings by polyelectrolytes (Wu et al., 2001; Sui and Schlenoff, 2003; Adamczyk et al., 2004), or the difference in the electro-osmotic flow in two different composite microchannels (Bianchi et al., 2001).

If the EDL can develop fully in the nanochannel, the electric potential reduces to zero in the center. However, if the ionic strength is low, the EDLs will overlap in the nanochannel and affect electro-osmosis, which is no longer plug flow but instead follows the electric potential $\psi(z)$ (Fig. 4). This phenomenon was first investigated by Burgreen and Nakache (1964), Levine, Marriott, and Robinson (1975), and complemented by Levine, Marriott, Neale, et al. (1975) in capillary slits, whereas Rice and Whitehead (1965) described flow in cylindrical capillaries. Under low ionic strength conditions, the electroosmotic velocity $v_{\mathrm{eo}}$ is dependent on the potential distribution $\psi(z)$ between two parallel plates [discussed below in Eq. (52)] and is given by

$$
v_{\mathrm{eo}}=-\frac{\varepsilon_{0} \varepsilon_{r} \zeta E_{x}}{\eta}\left(1-\frac{\psi(z)}{\zeta}\right) \text {. }
$$

(b) Electrophoresis. Electrophoresis is a common

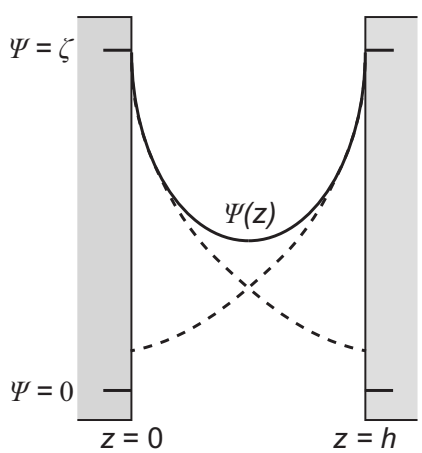

FIG. 4. Schematic representation of the potential distribution in a nanochannel with height $h$ in direction $z$ when the EDLs overlap (solid line), compared to the EDL potentials if the opposite wall is not present (dashed line). Adapted from Schoch, 2006.

technique used to move ions and molecules in microfluidic and nanofluidic channels (Oddy and Santiago, 2004). A simplified definition is that electrophoresis is the converse of electro-osmosis; the liquid is regarded as fixed, so that a molecule moves in the opposite direction relative to the solution. The complete description of electrophoresis has been given by Morgan and Green (2003), and is summarized here.

When a molecule is placed in an aqueous medium, the EDL screens the charges of the molecule, so that overall it is electroneutral and appears to have a zero net charge. Placing the molecule in an electric field results in its movement because ions in the EDL are not fixed to the surface. The counterions in the EDL are mobile and move in the direction opposite to that in which the molecule would move if it were unscreened. This ion movement gives rise to fluid motion around the molecule (electro-osmosis), and because of the viscosity of the aqueous medium, the fluid is stationary on a global scale. This results in ion movement as the molecule is pushed backward in the opposite direction.

Therefore, the electrophoretic mobility is dependent on the thickness of the electrical double layer and can be divided into categories of thin and thick EDLs, described by the product $\kappa a_{p}$ of the Debye-Hückel parameter $\kappa$ and the molecular radius $a_{p}$.

Thin electrical double layer, $\kappa a_{p} \gg 1$. For an EDL that is thin compared to the molecular radius, electrophoresis is the converse of electro-osmosis [Eq. (19)]. In this case, however, the surface moves relative to the stationary fluid, and the electrophoretic mobility is given by the Helmholtz-Smoluchowski limit,

$$
\mu_{\mathrm{ep}}=\frac{\varepsilon_{0} \varepsilon_{r} \zeta}{\eta} .
$$

Thick electrical double layer, $\kappa a_{p} \ll 1$. When a molecule has a thick EDL, the force acting on the molecule is the difference between the drag and Coulomb forces. The drag force is given by Stokes law $q E_{x}=f v_{\mathrm{ep}}$, leading to $\mu_{\mathrm{ep}}=v_{\mathrm{ep}} / E_{x}=q / f=q / 6 \pi \eta a_{p}$, where $f$ is the friction coefficient and $v_{\text {ep }}$ is the electrophoretic velocity. The $\zeta$ 
potential can be identified with the potential of a point charge as $\zeta=a_{p} E=a_{p} q / 4 \pi \varepsilon_{0} \varepsilon_{r}$. The combination of these expressions leads to the electrophoretic mobility in the Hückel-Onsager limit,

$$
\mu_{\mathrm{ep}}=\frac{2 \varepsilon_{0} \varepsilon_{r} \zeta}{3 \eta}
$$

The real situation is more complex than that described above, as co-ions around the molecule move ahead and counterions move in the direction opposite to the molecule. This results in a polarization field, which can increase or decrease the electrophoretic mobility. A second issue, which has not been considered, is the distortion of the field induced by movement of the molecule, known as the relaxation effect.

(c) Streaming potential. When a liquid is forced to flow through a small channel under hydrostatic pressure, ions in the mobile part of the EDL are carried downstream. This creates an electric current, which is called the streaming current, flowing in the same direction as the liquid. The accumulation of ions downstream sets up an electric field, which causes a current to flow in the opposite direction through the liquid (conduction current). When the conduction current is equal to the streaming current, steady state is achieved. The resultant electrostatic potential difference between the two ends of the channel is referred to as the streaming potential $\varphi$.

These properties are exploited to determine the $\zeta$ surface potential, which is done by either measuring the streaming potential (Horn and Onoda, 1978; Mockel et al., 1998; $\mathrm{Gu}$ and $\mathrm{Li}, 2000$ ) or measuring the streaming current directly (Jednacak and Pravdic, 1974). The streaming potential can also be measured with a nanoscale probe over the changes in the counterion concentration of the EDL. This concentration is dependent on the flow speed, and therefore results in a conductance change of the nanoprobe located in the EDL (Bourlon et al., 2007). In the case of EDL overlap in nanochannels, a correction factor for the streaming potential has to be considered (Burgreen and Nakache, 1964), and it has been reported that under such conditions the streaming potential has a constant value due to length-scale effects (Shim et al., 2007).

(d) Electroviscous effect. Whenever an electrical double layer at an interface in an ionic liquid is sheared, a potential difference in the form of a streaming potential is established, which will tend to resist the flow of liquid (Elton, 1948). This potential will produce a backflow by electro-osmosis, and the net effect is a diminished flow in the forward direction. The liquid appears to exhibit enhanced viscosity if its flow rate is compared to the flow without EDL effects. This phenomenon is denoted the electroviscous effect and is classified as follows (Hunter, 1981).

The primary electroviscous effect occurs in channels sufficiently large to allow complete development of the EDL and can be measured in microchannels (Ren et al., 2001; Vainshtein and Gutfinger, 2002).
The secondary electroviscous effect refers to an increase in the apparent viscosity of a fluid as a result of EDL interactions (Yang and Li, 1997). Tas et al. (2004) have observed that the capillary filling speed of demineralized water in 53-nm-high nanochannels is lower than expected from capillary action. This is attributed to the secondary electroviscous effect, and the apparent viscosity has been measured as being $24 \%$ higher than the bulk viscosity.

A real increase in the viscosity due to high electric fields in the neighborhood of a surface, for example, is referred to as the viscoelectric effect.

\section{Comprehensive electrokinetic theory for nanochannels}

It is important to consider aforementioned electrokinetic phenomena to develop a comprehensive transport model of molecules in nanochannels. Such processes have been described in the field of membrane science. In nanofiltration, the Teorell-Meyer-Sievers model can be applied; it assumes a uniform distribution of fixed charge, mobile ions, and electric potential (Hagmeyer and Gimbel, 1999; Bowen and Welfoot, 2002; GarciaAleman and Dickson, 2004). However, these assumptions are not applicable to membranes with pores containing a radius bigger than the Debye length, leading to the proposition of the space-charge model by Gross and Osterle (1968), and its development by Wang et al. (1995). The validity of this model to describe the electrokinetic phenomena in charged capillaries and transport through porous membranes has been confirmed (Szymczyk et al., 1999; Yaroshchuk et al., 2005). The space-charge model is an extension of the TeorellMeyer-Sievers model and is more calculation intensive, mathematically complicated, and computationally expensive, which makes it difficult to apply to solutions of mixed electrolytes. This led to the development of simplified approaches for modeling ion transport through porous membranes (Hawkins Cwirko and Carbonell, 1989; Smit, 1989; Basu and Sharma, 1997).

The space-charge model is one of the most complete physical interpretations of ion transport through nanometer-sized channels, which are assumed to be straight and bear a surface charge. The following equations are solved: (1) the Poisson equation for the distribution of the electric potential in nanochannels, (2) the Nernst-Planck equation for ion transport, and (3) the Navier-Stokes equations for laminar volumetric fluid flow. The Poisson and Nernst-Planck equations give ion distributions "beyond" the Poisson-Boltzmann [Eq. (6)] because they allow flows and other forces to drive ion fluxes. In the absence of other flows and forces, the Poisson and Nernst-Planck equations give the PoissonBoltzmann equation.

(a) Poisson equation and Boltzmann distribution. The electric potential is divided into two parts,

$$
\boldsymbol{\Phi}=\psi(x, z)+\varphi(x)
$$

where $\psi$ originates from the surface charge of the nanochannel and $\varphi$ is due to the streaming potential, 
with $x$ the longitudinal direction and $z$ the direction normal to the nanochannel surface. To calculate the electric potential in nanochannels, the following assumptions can be made since the nanochannels are much longer than they are high (Sasidhar and Ruckenstein, 1982). (1) The variations of the potential in $x$ are much smaller than in the $z$ direction. (2) The concentration variation in the $z$ direction at any $x$ position follows the equilibrium Boltzmann distribution, because normal ionic currents have to be compensated due to diffusion and electromigration within the EDL (Dukhin and Shilov, 1969),

$$
\mathbf{c}_{ \pm}=z_{\mp} c^{*} \exp \left(\mp z_{ \pm} e \psi / k_{B} T\right),
$$

where $c^{*}$ is the bulk concentration at negligible electrostatic interactions. With these two assumptions, the electric potential in nanochannels is given by the Poisson equation,

$$
\nabla^{2} \psi=-\frac{F}{\varepsilon_{0} \varepsilon_{r}} \sum_{i=1}^{2} z_{i} \mathbf{c}_{i},
$$

where $i=1$ indicates the cations and $i=2$ the anions.

(b) Nernst-Planck equation. The second equation is the extended Nernst-Planck equation, which accounts for the transport of charged molecules in the presence of an electric potential through the nanochannel. The total flux per area $\mathbf{J}_{i}$ of ion $i$ includes contributions from diffusional flux, migration of molecules in the electric field, and convective flux in the nanochannel,

$$
\mathbf{J}_{i}=-D_{i} \nabla \mathbf{c}_{i}-\frac{z_{i} F}{R T} D_{i} \mathbf{c}_{i} \nabla \boldsymbol{\Phi} \pm \mathbf{v}_{c} \mathbf{c}_{i},
$$

where $D_{i}$ is the diffusion coefficient of ion $i$ and $\mathbf{v}_{c}$ is the convective velocity field. The extended Nernst-Planck equation includes the contribution of convective flux, unlike the ordinary Nernst-Planck equation. Equation (26) is the sum of three terms, as follows:

- Fick's first law, which specifies the diffusion of molecules due to a chemical potential gradient (concentration gradient).

- The flux due to the electric potential $\mathbf{\Phi}$.

- Convective transport, which can be due to pressure, mechanical stirring, and external fields such as electric and magnetic fields (Fair and Osterle, 1970; van der Horst et al., 1995; Bowen et al., 1997; Garba et al., 1999). In nanopores, electro-osmotic transport due to an electric field has to be considered (Kemery et al., 1998; Bath et al., 2000; Bhattacharjee et al., 2001; Miller et al., 2001; Gasparac et al., 2003). The direction of $\mathbf{v}_{c}$ is dependent on the surface charge of the nanochannel, and is in the opposite direction from the first two contributions if the surface charge is negative.

(c) Navier-Stokes equations. The third contribution to the space-charge model is the Navier-Stokes equations, which represent a conservation of momentum in a differential form and are used to calculate $\mathbf{v}_{c}$. For incompressible Newtonian fluids, in which the viscosity is not dependent on shear stress (Gross and Osterle, 1968; Marshall and Mocskos, 1997), by neglecting inertia (Sasidhar and Ruckenstein, 1982), and assuming constant viscosity in the Navier-Stokes equations, the movement of fluid through channels is given by

$$
\begin{aligned}
& \nabla \mathbf{v}_{c}=0, \\
& -\nabla \mathbf{p}-F_{b} \sum_{i=1}^{2} z_{i} \mathbf{c}_{i} \nabla \psi-F_{b} \sum_{i=1}^{2} z_{i} \mathbf{c}_{i} \nabla \varphi+\eta \nabla^{2} \mathbf{v}_{c}=0,
\end{aligned}
$$

where Eq. (27) is the continuity equation, $\mathbf{p}$ is the external pressure, and $F_{b}$ is the body force. Equation (28) contains three different forces acting on any volume element inside the channel: (1) a mechanical force due to the internally generated pressure gradient (the first term), (2) a body force that develops because of the polarization of the EDL (caused by the concentration gradient through the nanochannel, the second term), and (3) a second body force caused by the streaming potential (the flow component due to the latter force is the electro-osmotic flow, the third term). All these forces (1)-(3) balance the viscous force generated by the convective motion of fluid [the fourth term in Eq. (28)].

The Navier-Stokes equations are usually solved with the assumption of zero relative fluid-solid velocity, called the no-slip boundary condition. However, observed flow rates in nanotubes exceeded values calculated from conventional hydrodynamics by more than three orders of magnitude (Majumder et al., 2005; Holt et al., 2006), demonstrating nearly frictionless flow. Parameters affecting such fluid slip are an interplay of surface roughness, dissolved gas, pressure, wetting, shear rate, and electrical properties (Neto et al., 2005; Squires and Quake, 2005; Lauga et al., 2006; Seth et al., 2007). We believe that further knowledge of liquid slip is important for nanofluidics because of the potential to design systems with enhanced fluid transport (Cheng and Giordano, 2002; Eijkel, 2007).

(d) Application to nanochannels. The space-charge model has been simplified by Pennathur and Santiago (2005a) to describe electrokinetic transport in long nanochannels, confirmed by their experimental studies (Pennathur and Santiago, 2005b). Assuming fully developed flow and negligible contributions from pressure gradients, they obtained the following expression for electrokinetic flow in nanochannels:

$$
\begin{aligned}
v_{\mathrm{eo}}+v_{\mathrm{ep}}= & \frac{\varepsilon_{0} \varepsilon_{r} \zeta E_{x}}{\eta W}\left\langle\exp \left(\frac{-z_{i} e\left[\psi(z)-\psi_{s}\right]}{k_{B} T}\right)\right. \\
& \left.\times\left(1-\frac{\psi(z)}{\zeta}\right)\right\rangle+z_{i} \mu_{\mathrm{ep}} E_{x},
\end{aligned}
$$

where $W=\left\langle\exp \left[-z_{i} e \psi(z) / k_{B} T\right]\right\rangle$ and \langle\rangle represents the cross-sectional averaged quantity. The exponential term in Eq. (29) describes the transverse distribution of the sample determined by analyte valence $z_{i}$, and the transverse potential distribution $\psi(z)$. The product of this exponential with $1-\psi(z) / \zeta$ captures the coupling between 


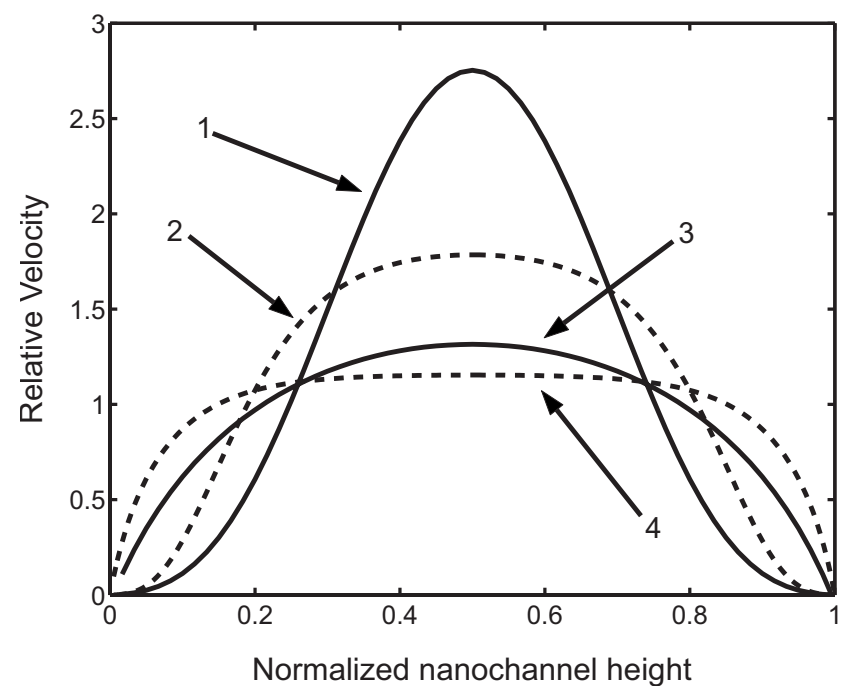

FIG. 5. Dimensionless species velocity due to electro-osmosis as a function of the normalized nanochannel height, for an anion in (1) 60- and (2) 200-nm-high channels, and for an uncharged molecule in (3) 60- and (4) 200-nm-high channels. Adapted from Garcia et al., 2005.

transverse electromigration and diffusion with streamwise flow.

Electrokinetic separation. The species transport velocity deduced from Eq. (29) shows that positively charged analyte molecules experience lower liquid velocities than negative sample ions, resulting in their separation in long nanochannels. This effect is illustrated as follows. Two like-charged objects repel each other due to Coulombic interactions, resulting in a segregation of negatively charged molecules toward the center of the likecharged nanochannel (Garcia et al., 2005; Griffiths and Nilson, 2006). On the contrary, positively charged analyte molecules equilibrate close to the negatively charged walls. There is no electrostatic effect on the concentration profile of neutral molecules. If the Debye length is comparable to the height $h$ of the nanochannel, then the electro-osmotic velocity profile follows the electric potential $\psi(z)$ (refer to Fig. 4), which has a maximum at the center of the channel and therefore leads to the separation of differently charged molecules in long nanochannels (Fig. 5). Garcia et al. (2005) found that the greatest difference in the maximum electrokinetic velocities is at $2 \lambda_{D} / h \sim 0.5$, because the extent of EDL overlap reduces the potential variation through the channel, and hence the development of electro-osmotic velocity profiles.

This phenomenology is described in Eq. (29), which shows that species velocity is dependent on the electrolyte mobility values, $\zeta$ potential, ion valence, and Debye length. Based on this analysis, Pennathur and Santiago (2005a) reported a method termed electrokinetic separation by ion valence, whereby both ion valence and ion mobility can be determined independently by comparing properties measured in microchannels and nanochannels. Additionally, it has been theoretically demonstrated that molecules differing in valence can be more

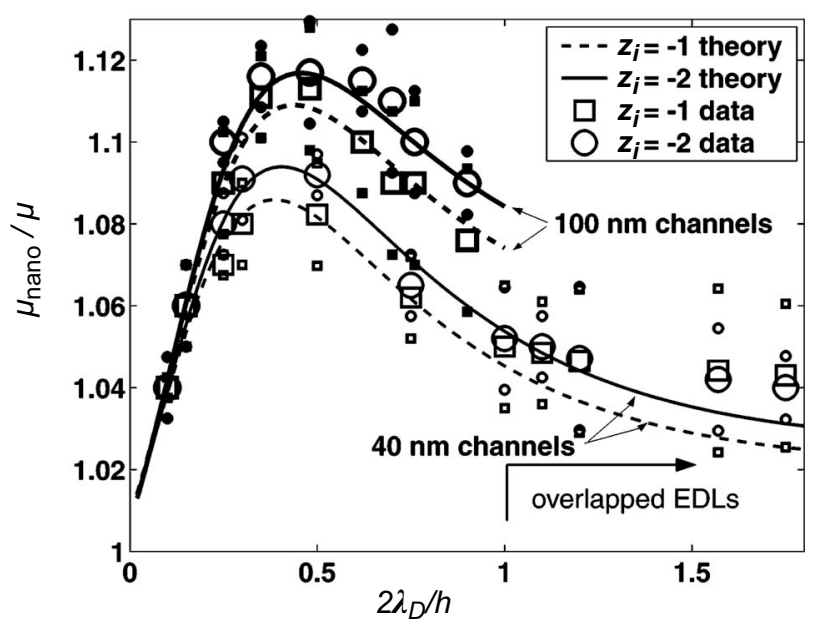

FIG. 6. Normalized mobility $\mu_{\text {nano }} / \mu$ as a function of $2 \lambda_{D} / h$ for two values of ion valence $z_{i}$. Measured normalized effective mobility in a nanochannel is compared with model predictions for both 40 and $100 \mathrm{~nm}$ channels. The large open symbols are the data, and the smaller, closed symbols are the corresponding error bars. Adapted from Pennathur and Santiago, $2005 b$.

efficiently separated in pressure-driven flow, while analytes with different mobility ratios should be separated in electric-field-driven flow (Xuan, 2007). This effect has been attributed to competitive effects of electro-osmosis and electrophoresis in nanochannels. For optimal electrokinetic separation by ion valence, $2 \lambda_{D} / h$ should be chosen between 0.3 and 0.5 (Fig. 6), and, to simplify the discussion of separations in long nanochannels, the definition of an analyte-specific length scale of each species has been proposed (Baldessari and Santiago, 2006).

Dispersion in nanofluidics. Velocity gradients across the channel cross section are central to dispersion, as they induce a shearing action (Taylor, 1953). Convective dispersion in microfluidic channels has been reviewed by Squires and Quake (2005); it is different for pressuredriven and electro-osmotic flow due to their parabolic and plug flow profiles, respectively (Griffiths and Nilson, 1999, 2006; Stein et al., 2006). In nanochannels, the previously mentioned electrokinetic effects increase the dispersion rate of counterions and decrease the rate of coions (De Leebeeck and Sinton, 2006). These authors also showed that the radially limited diffusion of ions by electromigration in response to the wall charge increases the dispersion of all ions relative to neutral species. Ions may diffuse radially only to the extent determined by the Boltzmann equilibrium distribution, whereas neutral species diffuse radially toward a uniform concentration distribution. It has been theoretically shown that dispersion is decreased in nanochannels because of induced electro-osmotic backflow (Xuan, 2007).

Electrokinetic energy conversion. The pressure-driven flow profile is parabolic and hence leads to ion separation in nanochannels, which can be exploited for electrokinetic energy conversion. Such a conversion of hydrostatic potential energy into electrical power has been investigated by van der Heyden et al. (2005, 2006), and 
this principle can be used for the development of micro fuel cells (Liu et al., 2005) or electrokinetic pumps (Min et al., 2004). The maximum energy conversion efficiency is obtained for $2 \lambda_{D} / h \sim 1$ (Morrison and Osterle, 1965; Daiguji, Yang, Szeri, et al., 2004) and has been measured to be $\sim 3 \%$ (van der Heyden et al., 2007), which is low compared to typical fuel cell efficiencies of $50 \%$.

\section{Electrode-electrolyte interface}

As an introduction to Sec. III.A about the electrical characterization of nanofluidic systems, we now describe how the electrode-electrolyte interface can be modeled by an electric equivalent circuit (Kovacs, 1994).

The interface between the electrode and the ionic solution can be represented by its capacitance $C_{\mathrm{EDL}}$, which is the capacitance sum of the Helmholtz layer $C_{H}$ and the Gouy-Chapman layer $C_{\mathrm{GC}}$,

$$
\frac{1}{C_{\mathrm{EDL}}}=\frac{1}{C_{H}}+\frac{1}{C_{\mathrm{GC}}} \text {. }
$$

The Helmholtz layer can be modeled as a simple capacitance,

$$
C_{H}=\frac{\varepsilon_{0} \varepsilon_{r} A}{d_{\mathrm{OHP}}},
$$

where $d_{\mathrm{OHP}}$ is the distance from the electrode to the outer Helmholtz plane (see Fig. 2). The capacitance of the Gouy-Chapman layer is obtained by differentiating Eq. (14),

$$
C_{\mathrm{GC}}=\frac{d \sigma_{s}}{d \psi_{s}}=\frac{\varepsilon_{0} \varepsilon_{r}}{\lambda_{D}} \cosh \left(\frac{z_{i} e \psi_{s}}{k_{B} T}\right) .
$$

\section{Charge-transfer resistance}

Modeling the electrode-electrolyte interface by capacitance does not describe the entire electric circuit. A resistive path must be considered in parallel to the capacitive path because a current can flow across the interface when a dc potential is applied. In order to define the resistive path in terms of the charge-transfer resistance $R_{t}$, we briefly discuss electrochemistry.

When a metal is placed in an electrolyte, chemical reactions will occur at the interface, whereby electrons are transferred between the metal and the ionic solution. This results in the formation of an electric field at the electrode surface, which affects further chemical reactions. Initially, the energy barrier for oxidation is lower than for reduction. As the reaction proceeds, excess electrons in the metal accumulate until the potential increases, which reduces the barrier for the reverse reaction (Kovacs, 1994). These two competing reactions eventually reach equilibrium, resulting in a zero net current across the interface. The absolute value of the current (per unit area) due to oxidation and reduction is referred to as the exchange current density $I_{d_{0}}$.

Thus, there is a constant flow of charge across the interface at equilibrium, but the net flow is zero. To achieve a flow of current, a net movement of charge is required, which is obtained when a potential $V_{a}$ is applied to push the total potential across the interface, away from its equilibrium value $V_{0}$. This potential difference $V_{a}-V_{0}$ leads to the total overpotential $\psi_{\text {to }}$, which is principally made from the sum of two overpotentials originating in the charge-transfer processes of the EDL leading to the charge-transfer overpotential $\psi_{\mathrm{ct}}$, and the diffusion of reactants to and from the electrode. For operation of an electrode near its equilibrium condition, the charge-transfer overpotential $\psi_{\text {ct }}$ dominates the overall current. As the potential moves further from equilibrium, the overpotential due to the diffusion of reactants may become a limiting factor.

The exchange current density $I_{d_{0}}$ is dependent on the material properties of the electrode, the electrolyte composition, and reactions taking place on the electrode. As the electrochemical reaction that dominates the exchange current is often unknown, the calculation of $I_{d_{0}}$ is difficult and is therefore experimentally determined by measuring the charge-transfer resistance $R_{t}$ around the equilibrium potential of the electrode in the electrolyte [see Eq. (33)]. The exchange current density $I_{d_{0}}$ is then used to calculate the electrical current density $I_{d}$ as a function of the charge-transfer overpotential $\psi_{\mathrm{ct}}$, as described by the Butler-Volmer equation (Park and Yoo, 2003),

$$
I_{d}=I_{d_{0}}\left[\exp \left(\frac{(1-v) z_{i} e \psi_{\mathrm{ct}}}{k_{B} T}\right)-\exp \left(\frac{-v z_{i} e \psi_{\mathrm{ct}}}{k_{B} T}\right)\right],
$$

where $v$ is the symmetry factor that reflects the energy barrier differences for oxidation and reduction reactions. To obtain an appreciable current density $I_{d}$, note that the exchange current density $I_{d_{0}}$ determines the magnitude of the charge-transfer overpotential $\psi_{\mathrm{ct}}$. Therefore, the appropriate choice of the electrode material affects the amount of current that flows in response to an applied potential. Since electrochemical reactions and gas evolution at the interface often occur at higher voltages, it is desirable to limit excursions from equilibrium as much as possible. Hence, electrode materials with a high exchange current density (like platinum) are desirable for integration into microfluidic and nanofluidic systems.

This leads to the definition of the charge-transfer resistance $R_{t}$ that appears in parallel to the interfacial capacitance, which under low-field conditions with $v=0.5$ (nonrectifying system) in the linear regime is given by

$$
R_{t}=\frac{k_{B} T}{z_{i} e I_{d_{0}}} \text {. }
$$

In summary, the model of a metal electrode placed in an electrolyte can be represented by a capacitance $C_{\mathrm{EDL}}$ in parallel to the charge-transfer resistance $R_{t}$. The relative impedance of these two elements determines the basic characteristics of the electrode. If the electrode has a high exchange current density, the charge-transfer resistance tends toward zero $\left(I_{d_{0}} \rightarrow \infty, R_{t} \rightarrow 0\right)$, as for 
$\mathrm{Ag} / \mathrm{AgCl}$ electrodes. Even though a significant current density flows across the interface, no substantial overpotential develops. This is referred to as an ideally nonpolarizable interface, and describes the optimum material in this case. The opposite situation $\left(I_{d_{0}} \rightarrow 0, R_{t} \rightarrow \infty\right)$ results in what is called an ideally polarizable interface. It behaves like a capacitance with no Faradaic processes occurring.

The charge-transfer overpotential $\psi_{\mathrm{ct}}$ has been presented above; it is important for small applied potentials $V_{a}$. If the magnitude of this potential is increased, reactants are not able to diffuse from the bulk to the interface fast enough, and the current becomes diffusion limited. This results in a diffusional impedance $Z_{W}$ in series with the charge-transfer resistance $R_{t}$, and $Z_{W}$ can be represented by either a series or parallel combination of resistance and capacitance. In 1899, Warburg proposed a model for this diffusional impedance $Z_{W}$, which is proportional to 1 over the square root of the frequency (DeRosa and Beard, 1977; McAdams et al., 1995).

\section{Constant phase element}

It is often experimentally observed (Metz, 2003; Gawad, 2004) that the impedance deviates from the purely capacitive behavior, that is expected theoretically for smooth and clean surfaces like that of liquid mercury. In such situations, the impedance spectrum $Z_{\mathrm{CPE}}$ is modeled by a constant phase element (CPE) (MacDonald, 1987), showing a power-law frequency dependence,

$$
Z_{\mathrm{CPE}}(\omega)=\frac{1}{C_{\mathrm{CPE}}(j \omega)^{n} \mathrm{CPE}},
$$

where $\omega$ is the frequency, $C_{\mathrm{CPE}}$ is the CPE capacitance, $n_{\mathrm{CPE}}$ is the CPE exponent, and $j$ is the imaginary unit. For perfectly smooth and clean electrodes, $n_{\mathrm{CPE}}$ is equal to unity, resulting in ideal capacitive behavior. However, if the electrode has a microscopic roughness due to scratches, pits, etc., the effective solution resistance varies along the surface, and the current density is inhomogeneous on a microscopic scale. This results in a CPE exponent in the range of $0.5<n_{\mathrm{CPE}}<1$.

The constant phase element obtained its name because the phase angle of $Z_{\mathrm{CPE}}$ is independent of the frequency, and has a value of $-\left(90 n_{\mathrm{CPE}}\right)$. It is a nonintuitive circuit element that was invented while looking at the response of whole systems (MacDonald, 1987).

\section{PHENOMENA AT THE MICROCHANNEL- NANOCHANNEL INTERFACE}

In order to investigate a nanochannel, it must be connected to the macroscopic world, which consequently leads to an interface between a nanochannel and typically a microchannel (see Sec. VI, Fig. 32). Different techniques can be used to probe a nanometer-sized opening, and we begin with a description of the electrical characterizations that reveal the unique properties of these channels. The electric field and fluid flow in a nanochannel can be substantially different from those in the connecting microchannel, resulting in large gradients at the geometric interface. These effects have been described in membrane science, where a porous membrane is in contact with a bulk solution, from which the Donnan potential is known and subsequently introduced. Then we present fluorescence measurements and modeling of the exclusion-enrichment effect in nanochannels at the diffusional limit and with external driving forces as electric fields and pressures. This phenomenon is generally described by partitioning at the microchannelnanochannel interface, which takes into account electrostatic and steric contributions. Finally, we provide insight into the complex process of concentration polarization at ion-selective interfaces.

\section{A. Electrical characterization of nanochannels}

Electrical measurements of nanochannels are performed because they do not require fluorescent labels and are integrable on a chip, which is favorable for potential applications. Furthermore, electrical characterizations of fluidic systems can readily be parallelized.

First, we focus on impedance spectroscopy to measure the nanochannel conductance. It has been shown that a simple physical model for ion transport through a nanochannel can explain its conductance value at varying ionic strengths. Indeed, we see that a conductance plateau (on a log-log scale) is obtained at low ionic strength when the Debye length becomes comparable to the characteristic dimension of the nanochannel. This effect is explained by an excess of mobile counterions in the nanometer-sized aperture, which equilibrate the surface charge to maintain electroneutrality. Subsequently, we demonstrate that ion flow can be regulated with chemical surface modifications and field-effect modulations.

A nanochannel can be distinguished from a nanopore by its length, as the nanochannel length is much longer than the nanometer characteristic dimension of the opening, whereas a nanopore has a length approximately within an order of magnitude of its minimal opening dimension. We show that if a nanopore or nanochannel has an asymmetric geometry or surface charge distribution along its longitudinal axis, ionic current rectification is obtained, as in a diode.

\section{Impedance spectroscopy}

To electrically characterize nanochannels that are interfaced with microchannels, we recommend the placement of electrodes as close as possible to the nanochannel openings in order to increase the sensitivity (Schoch, 2006). Therefore, the electrodes have to be integrated into the microchannels, which can be achieved with standard microfabrication techniques. Platinum electrodes were used by Schoch et al. (2005), because this material has a low chemical reactivity and hence will not be reduced or oxidized when electric potentials are applied. However, the platinum electrodes bear an overpotential, 
and when an electric potential is applied, this results in a start-up current peak and a decay with the square root of time, as described by chronoamperometry and the Cottrell equation (Girault, 2004),

$$
I(t)=n F A c_{R} \sqrt{\frac{D_{R}}{\pi t}}
$$

where $n$ is the number of electrons transferred per molecule, $A$ is the electrode surface, $c_{R}$ is the concentration of the reduced species $R$, and $D_{R}$ is the diffusion coefficient of the reduced species $R$. Equation (36) describes the fact that the current $I$ induces oxidation and reduction at the electrodes, and therefore the formation of the gases $\mathrm{O}_{2}$ and $\mathrm{H}_{2}$. After a few seconds, this results in a diffusion-limited current because generation of hydrogen and oxygen can only occur at the same rate as the diffusion of the deposition products into solution. Natural convection prevents the current from dropping to zero in reality, leading to drifting currents.

These problems can be overcome by performing ac instead of dc measurements. When the current flow changes its direction each half-cycle of the sinusoidal applied voltage, the system can be regarded as symmetrical. Diffusion-limited currents cannot occur and chemical reactions are not induced because of the alternating electric field (Asbury and Engh, 1998).

The electrical properties of materials and their interfaces with electrodes can be characterized with impedance spectroscopy (MacDonald, 1987). This method can be used to investigate the dynamics of bound or mobile charges in the bulk or interfacial regions of any kind of solid or liquid material. Impedance spectroscopy involves relatively simple electrical measurements that can readily be automated, the results of which may often be correlated with many complex material variables. Generally, the most common and standard approach is to measure the impedance directly in the frequency domain by applying a single-frequency voltage to the interface and measuring the phase shift and amplitude, or real and imaginary parts, of the resulting current at that frequency.

Impedance spectroscopy has only recently been used to describe nanometer-characteristic openings. Yi et al. (2005) demonstrated the detection of DNA molecules in a nanogap due to permittivity changes, and IonescuZanetti et al. (2006) analyzed sensitivity to sample permittivity changes. They reported the detection limit of the impedance to be a $1.7 \%$ change in permittivity, showing the promise of nanogaps for label-free biomolecule detection on platforms amenable to highthroughput configurations. The impedance imaging of individual pores in a porous membrane has been demonstrated by Ervin et al. (2005, 2006) using a lowimpedance bypass channel around the membrane to shunt the ion current. Cannon et al. (2003) analyzed the resistances of individual fluidic elements on their device, which consisted of two microchannels connected via a nanoporous membrane in a multilayer microfluidic architecture. Polymer electrolytes confined to a porous

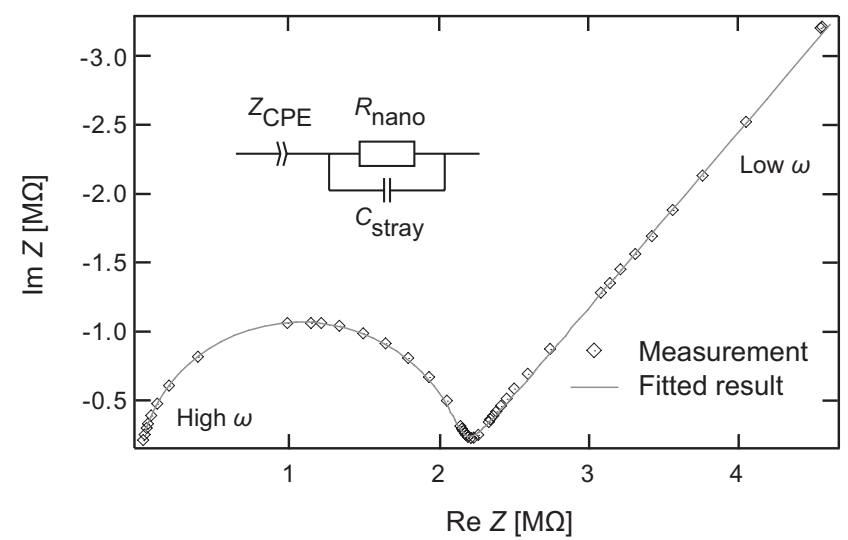

FIG. 7. Complex impedance plot of 50-nm-high nanochannels measured in a $0.1 M \mathrm{KCl}$ solution for frequencies $\omega$ from $20 \mathrm{~Hz}$ to $1 \mathrm{MHz}$. A value of $2.22 \mathrm{M} \Omega$ has been obtained for the nanochannel resistance. The inset shows the electric equivalent circuit modeling the EDL on the platinum electrodes, consisting of the nanochannel resistance $R_{\text {nano, }}$, the stray capacitance of the chip $C_{\text {stray }}$, and the impedance of the constant phase element $Z_{\mathrm{CPE}}$. Adapted from Schoch et al., 2005.

polycarbonate membrane were exposed by Layson and Teeters (2004) to different temperature and humidity conditions, and impedance spectroscopy measurements allowed deeper insight into the changes of the polyelectrolytes. Terrettaz et al. (2003) used impedance spectroscopy on planar gold substrates to monitor ion-channel protein activity modulations by the selective binding of an antibody.

Schoch (2006) performed impedance spectroscopy measurements of nanochannels interfaced by two microchannels containing microfabricated platinum electrodes at different ionic strengths to reveal their properties. A measurement from 50-nm-high nanochannels filled with $0.1 M \mathrm{KCl}$ is presented in Fig. 7. The semicircle in the complex impedance plot is due to the resistance of the nanochannel $R_{\text {nano }}$ in parallel to the stray capacitance $C_{\text {stray. }}$ The effective electric resistance of the nanochannels corresponds to the high intercept point of the extrapolated semicircle with the real axis (Park and Yoo, 2003; Li, Yu, Harrell, et al., 2004), resulting in $R_{\text {nano }}$ $=2.22 \mathrm{M} \Omega$ at $4 \mathrm{kHz}$. At high frequency $\omega$, the impedance is affected by the stray capacitance of the chip, and since ions cannot follow the electric field, this results in an impedance tending toward zero. In the complex impedance plot, the straight line at low frequency $\omega$ is due to the EDL on the electrodes dominating the measurement. The impedance of the EDL is represented by the constant phase element $Z_{\mathrm{CPE}}$ (see Sec. II.C.2), which is commonly favored over the use of a simple capacitor (Gawad, 2004).

\section{Nanochannel conductance}

(a) Mathematical model. For the electrical modeling of a nanochannel filled with a 1:1 electrolyte such as $\mathrm{KCl}$, the conductivity of the bulk solution $\gamma_{\text {bulk }}$ has to be considered (Morgan and Green, 2003), 


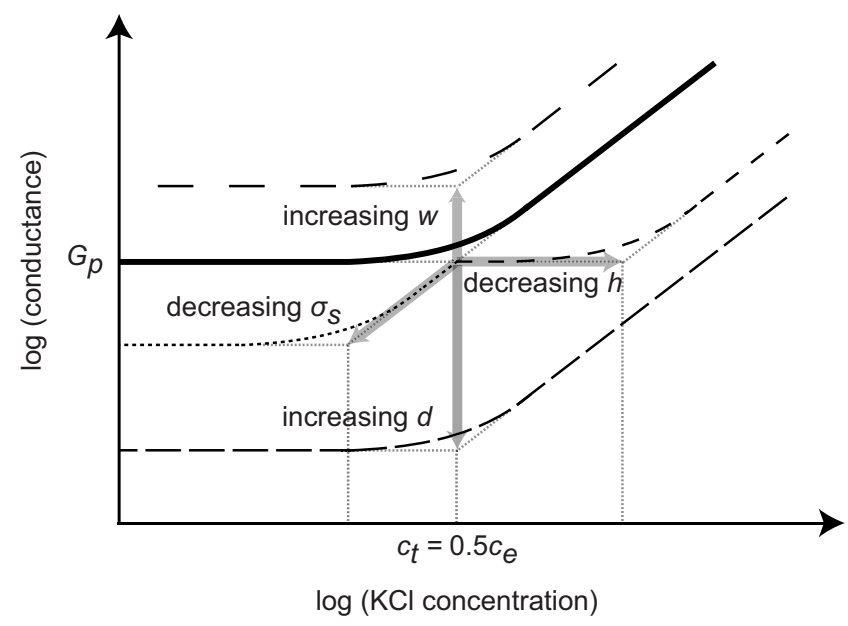

FIG. 8. Schematic behavior of the nanochannel conductance as a function of the $\mathrm{KCl}$ concentration. The impact of the characteristic surface charge density $\sigma_{s}$, the width $w$, the length $d$, and the height $h$ of the nanochannel on its conductance is shown. Below the transition $\mathrm{KCl}$ concentration $c_{t}$, a conductance plateau $G_{p}$ is reached, where $c_{t}$ corresponds to 0.5 times the excess mobile counterion concentration $c_{e}$. From Schoch and Renaud, 2005.

$$
\gamma_{\mathrm{bulk}}=e \sum_{i} \mu_{i} n_{i}=\left(\mu_{\mathrm{K}^{+}}+\mu_{\mathrm{Cl}^{-}}\right) n_{\mathrm{KCl}} e
$$

where $\mu_{i}$ is the mobility and $n_{i}$ is the volume density of ions $i$. At high ionic strength, Eq. (37) can be used to calculate the conductance of a nanochannel, but at low ionic strength, a conductance plateau (on a log-log scale) is observed (see Fig. 8) (Schoch and Renaud, 2005). To explain this effect, we consider the requirement for overall electroneutrality since the surface charge density must be balanced by counterions, which are present in the nanometer-sized aperture at the excess mobile counterion concentration (Daiguji, Yang, and Majumdar, 2004)

$$
c_{e}=\frac{2 \sigma_{s}}{h N_{A} e}
$$

where $\sigma_{s}$ is the surface charge density in the Stern layer and the diffuse double layer, and $N_{A}$ is the Avogadro constant. When the bulk salt concentration is lower than $c_{e}$, the nanochannel attracts $\mathrm{K}^{+}$ions to satisfy the electroneutrality condition, whereas $\mathrm{Cl}^{-}$ions are electrostatically repelled from the opening. Therefore, excess counterions in the nanometer-sized aperture explain the conductance plateau (on a log-log scale) at low ionic strength. A simplified model of the nanochannel conductance $G$ for all $\mathrm{KCl}$ concentrations is obtained through the superimposition of the bulk conductance and the excess counterion conductance (Schoch and Renaud, 2005),

$$
G=\left(\mu_{\mathrm{K}^{+}}+\mu_{\mathrm{Cl}^{-}}\right) n_{\mathrm{KCl}} e \frac{w h}{d}+2 \mu_{\mathrm{K}^{+}} \sigma_{s} \frac{w}{d},
$$

where $w$ is the width and $d$ is the length of the nanochannel. The conductance in a nanochannel for ionic strengths higher than the excess mobile counterion concentration $c_{e}$ is therefore dependent on channel geometry [first term in Eq. (39)]. The second term illustrates that, for electrolyte concentrations lower than $c_{e}$, the conductance is dominated by $\sigma_{s}$ and independent of the height $h$ of the nanochannel. When the first and second terms of Eq. (39) are equal, the transition $\mathrm{KCl}$ concentration $c_{t}$ results, below which the conductance plateau $G_{p}$ (on a log-log scale) is reached (see Fig. 8).

(b) Validations. Chemical surface modifications such as $p \mathrm{H}$ adjustment of the solution and polymer coatings have confirmed the modification of surface charge density in nanochannels (Stein et al., 2004; Schoch and Renaud, 2005). The aforementioned model has been applied by Karnik, Castelino, Fan, et al. (2005) to demonstrate two effects: surface charge changes after adsorption of biomolecules, and occlusion of nanochannels due to the immobilization of streptavidin and biotin on the walls of the channel.

Electrolyte transport studies on synthetic nanopores with diameters between 1 and $3 \mathrm{~nm}$ have supported the evidence of higher pore conductivities than the bulk conductivity for dilute salt concentrations, because the Debye length and the van der Waals radius of the ions become comparable to the pore radius (Ho et al., 2005). For pores with diameters of a few nanometers, molecular-dynamics simulations have been used to estimate ion mobility, which is affected by confinement and ion-surface interactions.

(c) Considerations. It is known from the literature that the absolute value of the $\zeta$ potential increases with dilution (Erickson et al., 2000; Gu and Li, 2000; Kirby and Hasselbrink, 2004), which results in a higher number of attracted counterions near the surface, neutralizing the fixed surface charge. This leads to a decrease in the fixed surface charge density with decreasing ionic strength. In relation to the conductance plateau on a log-log scale, there are two competing factors at low ionic strength: the higher $\zeta$ potential increases the concentration of counterions near the surface, which implies an increase in the nanochannel conductance, and the reduced fixed surface charge density leads to a decrease in the conductance $G$. In Eq. (39), $\sigma_{s}$ is defined as the charge density in the Stern layer and the diffuse double layer, affecting both previously mentioned factors, resulting in a saturation of the nanochannel conductance at low ionic strength.

A phenomenon similar to these two competing effects is denoted as charge regulation (Ninham and Parsegian, 1971; Healy et al., 1980); it states that the charge densities of two objects are functions of their separation distances, and has been discussed by Behrens and Borkovec (1999) and Behrens and Grier (2001). To consider contributions from both the surface charge density and 
the salt concentration, Tessier and Slater (2006) proposed an effective Debye length, which enables researchers to solve systems in osmotic equilibrium with an electrolyte reservoir.

When electrolyte solutions are subjected to an electric field, ion-ion interactions have to be considered due to the combined action of the electrophoretic effect and relaxation in the ionic atmosphere (Heng et al., 2005). This leads to a dependence on the molar conductivity of an electrolyte as a function of its concentration, which is described in the empirical Kohlrausch law (Girault, 2004),

$$
\Lambda_{m}=\Lambda_{m}^{0}-\alpha \sqrt{c}
$$

where $\Lambda_{m}$ is the molar conductivity, $\Lambda_{m}^{0}$ is the limiting molar conductivity in dilute electrolyte solutions where ion-ion interactions can be neglected, and $\alpha$ is a constant. Such effects were not considered in Eq. (39), which captures only the most relevant physical effects.

\section{Ionic current rectification}

The previously mentioned nanochannels are symmetric, and their measured currents for voltages of the same amplitude but opposing polarities have similar absolute values. However, asymmetric pore geometries or surface charge discontinuities in a nanopore lead to nonlinear, diodelike voltage-current curves at symmetric electrolyte conditions. Such ionic current rectification in nanofluidic systems was first reported for quartz nanopipet electrodes (Wei et al., 1997). Siwy et al. (2002) studied rectification with conically shaped nanopores in polymer membranes, as described hereafter. Additionally, these synthetic nanopores show voltage gating, like biological ion channels (Sec. V.A.1), because of carboxylate groups at the surface, formed during the nanopore fabrication with the track etching technique (Sec. VI.F).

The poorly defined nature of the chemistry and charge of the polymeric pores has limited the understanding of the current-rectification function; coating the nanopore walls with a gold layer has offered deeper insight (Siwy et al., 2004). A judicious choice of the electrolyte and $p \mathrm{H}$ value adjustments has demonstrated that rectification requires surface charge. The electric potential inside conical nanopores has been calculated with the shape of an asymmetric sawtooth (see Fig. 9), which allows ionic current rectification to be explained by the ratchet mechanism (Ajdari and Prost, 1992; van Oudenaarden and Boxer, 1999; Gauthier and Slater, 2003; Matthias and Muller, 2003). Cation-selective conical nanopores rectify ionic current with the preferential direction of cation flow from the narrow entrance to the wide opening of the pore (Siwy and Fulinski, 2004), strong enough to pump ions against their concentration gradient (Siwy and Fulinski, 2002a).

The magnitude of ionic current rectification depends on the ionic strength, and the diodelike behavior becomes more pronounced with dilution because the depth of the asymmetric ratchet tooth increases. The rectification ratio is defined as the ratio of currents recorded for

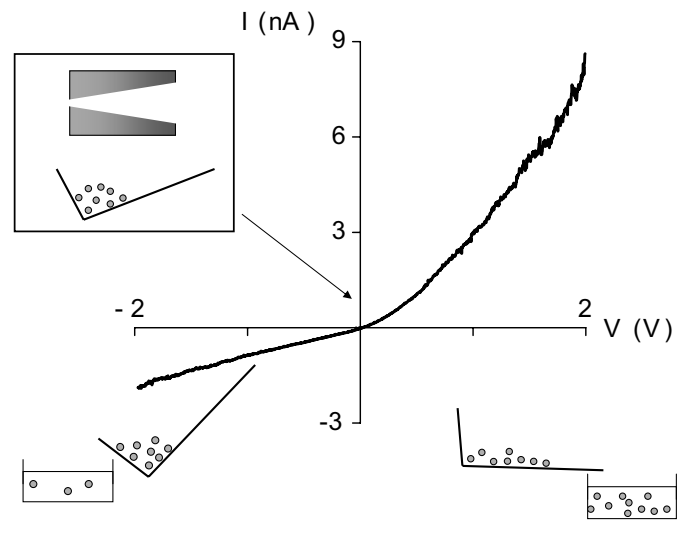

FIG. 9. Voltage-current curve showing ionic current rectification of conical nanopores, explained with the ratchet mechanism. The electric potential in conical nanopores takes the form of an asymmetric tooth, which turns when the external potential $V$ is changed. For positive voltages, the force to move ions through the pore is smaller than for negative voltages, when ions are trapped inside the pore. The amount of current transported by cations or anions can be calculated from such voltage-current curves. From Siwy and Fulinski, 2004.

voltages of similar amplitude but opposite polarities, and it has been reported that this ratio versus the ionic strength is maximal at moderate electrolyte concentrations for nanopores contained in polymer membranes (Cervera et al., 2006). Since this maximum has not been observed for gold nanopores, it is speculated that the "dangling ends" of the polymers could act as electromechanical gates, changing the pore diameter for different applied voltages (Schiedt et al., 2005).

An asymmetry in the electric potential cannot be obtained solely with conical shapes of nanopores with uniformly charged walls. Karnik et al. (2007) demonstrated ionic current rectification using symmetric nanochannels whose walls are coated with positively and negatively charged regions, and hence bear an asymmetric surface charge distribution. Such patterned discontinuities can be achieved in the axial direction by controlling the position of the reaction front, which is possible because the time for ion diffusion across the channel is much shorter than the transit time of ions through the channel (Kwak and Hasselbrink, 2005). This leads to a self-aligning process denoted by diffusion-limited patterning, which can be used to coat multiple species with spatial resolution better than $1 \mu \mathrm{m}$ (Karnik, Castelino, Duan, et al., 2006).

The highest ionic current rectification ratios have been obtained when the above-described rectification effects were combined by positively and negatively patterning charged regions in conical nanopores (Vlassiouk and Siwy, 2007). The operation mechanism of such nanofluidic diodes is explained by the fact that, when a voltage of opposite polarity with respect to the patterned regions is applied through the nanopore, EDL concentration profiles of anions and cations overlap and high ionic currents result. Reversing the voltage moves cations and anions toward the bulk, leading to a depletion zone inside the nanopore, and therefore low ionic cur- 
rents. These rectification properties are similar to those of bipolar semiconductor diodes or ion-exchange bipolar membranes (Mafe and Ramirez, 1997). Furthermore, modeling has revealed that tunable ion current rectification in nanopores is achievable with two thin layers of opposite doping, forming a semiconductor membrane (Gracheva et al., 2007).

Moreover, ionic selectivity is not solely a property of the nanopore itself, but also depends on the direction of the concentration gradient. Using a model based on the Poisson and Nernst-Planck equations, Cervera et al. (2007) quantitatively described the reversal potential in conical nanopores, which depends on the direction of the applied ionic strength gradient. Such rectification of ion transport through a concentration gradient has also been shown in symmetric nanochannels (Cheng and Guo, 2007).

\section{B. Donnan equilibrium}

A charge-selective nanochannel and an adjacent electrolyte solution may well be in equilibrium, even if the electric potentials or the concentrations the two systems are different, as long as the differences compensate for each other, and the Gibbs free energy of both systems is identical. This electrochemical equilibrium is referred to as the Donnan equilibrium. It is obtained when an electric potential difference between two systems is compensated by a concentration difference of ions (Donnan, 1924, 1995). The Donnan equilibrium has been developed for porous membranes in contact with a bulk solution, and we present it here because it is relevant for nanochannels interfaced with microchannels.

\section{Donnan potential}

When a charge-selective channel is in equilibrium with an adjacent electrolyte solution, the electrochemical potentials $\tilde{\mu}_{i}$ of the permeating cation or anion $i$ are equal on sides I and II (see Fig. 10) (Rieger, 1994; Hamann et al., 1998; Bockris and Reddy, 2000),

$$
R T \ln \left(c_{i}^{\mathrm{I}} / c^{0}\right)+z_{i} F \Phi^{\mathrm{I}}=R T \ln \left(c_{i}^{\mathrm{II} /} c^{0}\right)+z_{i} F \Phi^{\mathrm{II}} .
$$

Equation (41) holds for constant pressure, temperature, and an ideal solution, which means that the activity of a component is identical to its concentration. This equation can be solved for the electric potential difference $\Phi_{D}=\Phi^{\mathrm{II}}-\Phi^{\mathrm{I}}$,

$$
\Phi_{D}=\frac{R T}{z_{i} F} \ln \left(\frac{c_{i}^{\mathrm{I}}}{c_{i}^{\mathrm{II}}}\right) .
$$

The potential $\Phi_{D}$ is denoted the Donnan potential, and results in the Donnan ratio $r_{D}$ of cations $\left(i=\mathrm{ca}, z_{i}\right.$ $=1)$ and anions $\left(i=\right.$ an,$\left.z_{i}=-1\right)$ between sides I and II,

$$
r_{D}=\left(\frac{c_{i}^{\mathrm{I}}}{c_{i}^{\mathrm{II}}}\right)^{1 / z_{i}}=\frac{c_{\mathrm{ca}}^{\mathrm{I}}}{c_{\mathrm{ca}}^{\mathrm{II}}}=\frac{c_{\mathrm{an}}^{\mathrm{II}}}{c_{\mathrm{an}}^{\mathrm{I}}} .
$$

Setting up Eq. (41) for a 1:1 electrolyte, once for cations and once for anions and summing the two, results in (a)

I II

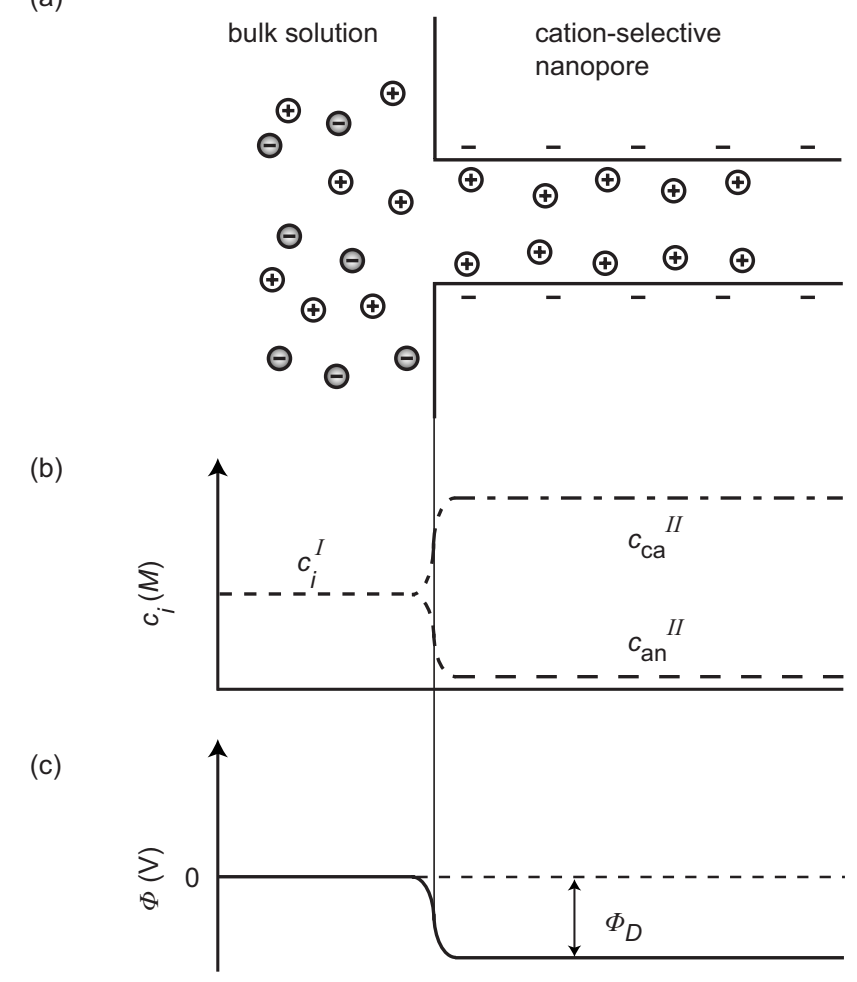

FIG. 10. Donnan equilibrium at the microchannelnanochannel interface. (a) Schematic drawing presenting a bulk solution (I) and an adjacent cation-selective nanochannel (II). (b) Concentrations $c_{i}^{\mathrm{I}}$ of cations and anions in the bulk solution are equal, whereas in the permselective nanochannel the concentration of cations $c_{\mathrm{ca}}^{\mathrm{II}}$ is higher than the concentration of anions $c_{\mathrm{an}}^{\mathrm{II}}$. (c) The Donnan potential $\Phi_{D}$ is negative in the cation-selective nanochannel. From Schoch, 2006.

$c_{\mathrm{ca}}^{\mathrm{I}} c_{\mathrm{an}}^{\mathrm{I}}=c_{\mathrm{ca}}^{\mathrm{II}} c_{\text {an }}^{\mathrm{II}}$. The cation concentration inside the nanochannel is $c_{\mathrm{ca}}^{\mathrm{II}}=c_{w}^{\mathrm{II}}+c_{\mathrm{an}}^{\mathrm{II}}$, where $c_{w}^{\mathrm{II}}$ is the wall charge concentration averaged over the channel volume. From these two equations and Eq. (43), the concentrations of cations and anions in the nanochannel are calculated to be (Sata, 2004)

$$
\begin{aligned}
& c_{\mathrm{ca}}^{\mathrm{II}}=0.5 \sqrt{4\left(c_{i}^{\mathrm{I}}\right)^{2}+\left(c_{w}^{\mathrm{II}}\right)^{2}}+c_{w}^{\mathrm{II}}, \\
& c_{\mathrm{an}}^{\mathrm{II}}=0.5 \sqrt{4\left(c_{i}^{\mathrm{I}}\right)^{2}+\left(c_{w}^{\mathrm{II}}\right)^{2}}-c_{w}^{\mathrm{II}} .
\end{aligned}
$$

The Donnan ratio shows that exclusion of co-ions and enrichment of counterions results in a cation-selective nanopore (see Fig. 10); this behavior is exploited in membrane science for filtration purposes. The Donnan effect can be enhanced using species with high ion valence (Chaufer et al., 1996; Yaroshchuk, 2001b) or specific surface coatings (Hollman and Bhattacharyya, 2004).

\section{Membrane potential}

The membrane potential has been developed in the context of ion-exchange membranes, and we present it here because charge-selective nanochannels have similar characteristics to permselective membranes. Ion- 


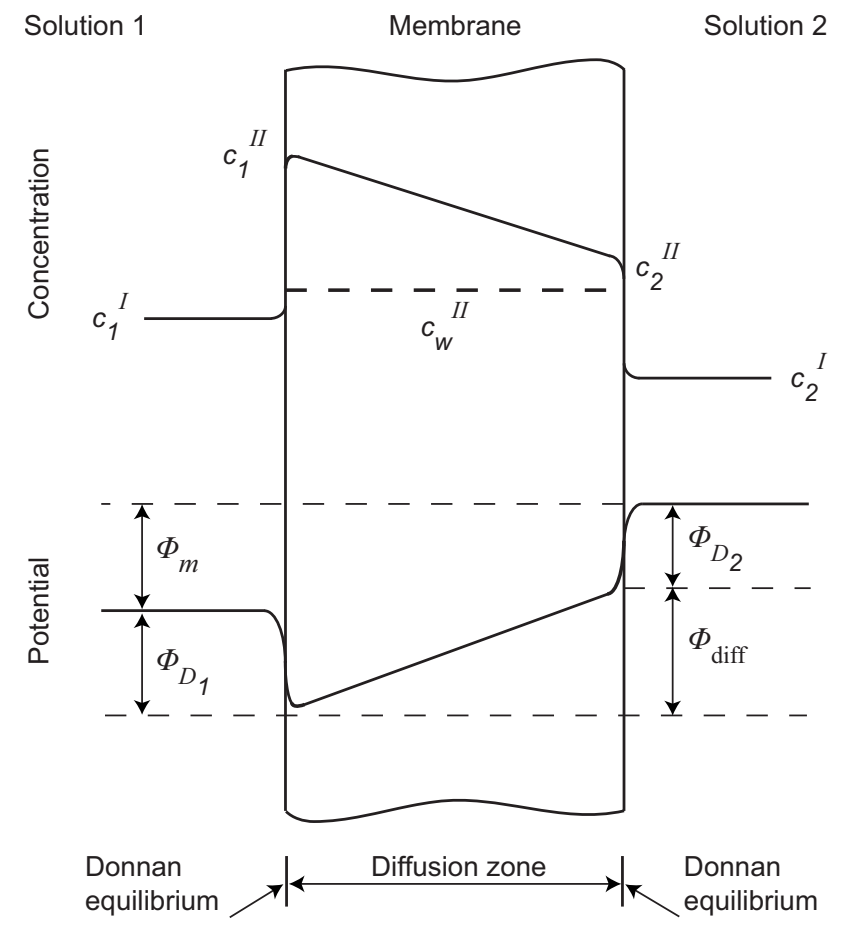

FIG. 11. Schematic representation of the membrane presenting the concentration profile and resulting potential differences for a permselective membrane, separating solution 1 from solution 2 . The concentrations in solution 1 are $c_{1}^{\mathrm{I}}$ in the bulk and $c_{1}^{\mathrm{II}}$ at the membrane surface, and in solution 2 they are $c_{2}^{\mathrm{I}}$ in the bulk and $c_{2}^{\mathrm{II}}$ at the membrane surface. The wall charge concentration averaged over the channel volume is $c_{w}^{\mathrm{II}}$. The membrane potential $\Phi_{m}$ is composed of the Donnan potential $\Phi_{D_{1}}$ in solution 1 , the Donnan potential $\Phi_{D_{2}}$ in solution 2, and the diffusion potential $\Phi_{\text {diff }}$. Adapted from Sata, 2004.

exchange membranes are important tools for separation processes, which can be classified into mass separation, chemical synthesis, and energy conversion and storage processes (Morrison and Osterle, 1965; Strathmann, 2004).

If two solutions of different ionic concentrations are separated by a permselective membrane, a membrane potential $\Phi_{m}$ is created (Fig. 11). The membrane potential can be measured directly, whereas the Donnan potential is usually calculated. $\Phi_{m}$ is composed of the two Donnan potentials $\Phi_{D_{1}}$ in solution 1 and $\Phi_{D_{2}}$ in solution 2 and the diffusion potential $\Phi_{\text {diff }}$, caused by differences in the transference numbers of different ions in the membrane,

$$
\Phi_{m}=\Phi_{D_{1}}+\Phi_{D_{2}}+\Phi_{\text {diff }} .
$$

When the Donnan equilibrium of counterions is attained at the membrane interface, this leads to the Donnan potential at interface 1 ,

$$
\Phi_{D_{1}}=\frac{R T}{F} \ln \frac{c_{1}^{\mathrm{II}}}{c_{1}^{\mathrm{I}}}=\frac{R T}{F} \ln \frac{\sqrt{4\left(c_{1}^{\mathrm{I}}\right)^{2}+\left(c_{w}^{\mathrm{II}}\right)^{2}}+c_{w}^{\mathrm{II}}}{2 c_{1}^{\mathrm{I}}} .
$$

At interface 2, the Donnan potential is described by

$$
\Phi_{D_{2}}=\frac{R T}{F} \ln \frac{c_{2}^{\mathrm{I}}}{c_{2}^{\mathrm{II}}}=\frac{R T}{F} \ln \frac{2 c_{2}^{\mathrm{I}}}{\sqrt{4\left(c_{2}^{\mathrm{I}}\right)^{2}+\left(c_{w}^{\mathrm{II}}\right)^{2}}+c_{w}^{\mathrm{II}}} .
$$

The diffusion potential has a maximum value for a completely permselective membrane, and the membrane potential is in this case the sum of the two Donnan potentials. When the membrane is not completely permselective, the diffusion potential is calculated as

$$
\Phi_{\text {diff }}=-\frac{t_{\mathrm{ca}}^{\mathrm{II}}}{z_{\mathrm{ca}}} \frac{R T}{F} \ln \frac{c_{2_{\mathrm{ca}}^{\mathrm{I}}}^{\mathrm{I}}}{c_{1_{\mathrm{ca}}}^{\mathrm{I}}}+\frac{t_{\mathrm{an}}^{\mathrm{II}}}{z_{\mathrm{an}}} \frac{R T}{F} \ln \frac{c_{2_{\mathrm{an}}^{\mathrm{I}}}^{\mathrm{I}}}{c_{1_{\mathrm{an}}}^{\mathrm{I}}},
$$

where $t_{\mathrm{ca}}^{\mathrm{II}}$ and $t_{\mathrm{an}}^{\mathrm{II}}$ are the transference numbers of cations and anions (the fraction of the current transported by the appropriate ion type) and $z_{\mathrm{ca}}$ and $z_{\mathrm{an}}$ are the valences of cations and anions, respectively.

\section{Exclusion-enrichment effect}

Section III.A.2 discussed the excess of counterions present inside nanochannels at low ionic strength. This enrichment of counterions in and exclusion of co-ions from a nanometer-sized opening due to electrostatic interactions with the surface charge is called the exclusionenrichment effect (EEE), described by Plecis et al. (2005). Subsequently, we discuss the mathematical model of this phenomenon.

The consequences of the exclusion-enrichment effect on passive transport of charged species through a nanochannel can be investigated by measuring the quantitative changes in the nanochannel permeability when the Debye length increases. For anions, the decrease in nanochannel permeability at low ionic strength can be schematically described as electrostatic "narrowing" of the nanometer-sized aperture, in analogy to an aperture known from optics (see Fig. 12). At high ionic strength, the instantaneous flux $\phi^{*}$ is proportional to the geometric cross section $S^{*}$. When the EDL gets larger at low ionic strength, the effective cross section $S_{\text {eff }}$ through which anions can diffuse is reduced, resulting in a lower effective flux $\phi_{\text {eff }}$. For cationic species, the enrichment effect at low ionic strength increases the number of cations in the nanochannel that can be transported by diffusion. This results in a higher effective flux.

\section{Permselectivity of a nanochannel}

The permselectivity induced by the EEE in nanoporous structures was first experimentally investigated with the advent of nanoporous membranes. Many groups established the possibility of modulating the transport of charged species through such synthetic sieves. A gold-plating technique was developed that allowed for control of both the size and surface chemistry of pores on polycarbonate track-etched membranes (Nishizawa et al., 1995; Lee and Martin, 2001; Martin et al., 2001). Additional transport studies found a higher permselectivity of these membranes on anions or cations when the Debye length was on the order of the pore radius. The ionic permselectivity was also shown to de- 

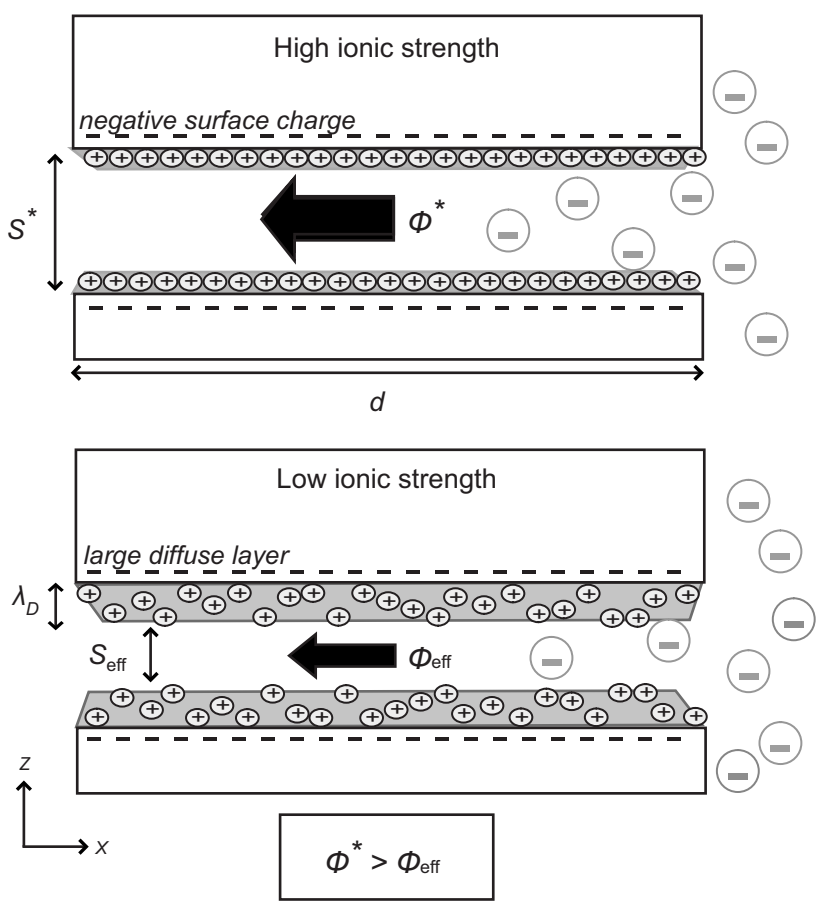

FIG. 12. Schematic of the exclusion effect on anionic transport. At high ionic strength, the Debye length is small so that anions (co-ions) can freely diffuse through the whole cross section $S^{*}$ of the nanochannel. The instantaneous flux without electrostatic interactions $\phi^{*}$ is proportional to $S^{*}$. The Debye length increases with dilution, reducing the effective cross section $S_{\text {eff }}$ through which anions can diffuse. This results in a lower effective flux $\phi_{\text {eff. }}$ Adapted from Plecis et al., 2005.

pend on the surface charge. Positively charged pores showed increased anion flux compared to negatively charged pores, and the inverse for cations, which legitimizes the idea that the EEE is responsible for this permselectivity. Numerical solutions of the fundamental flux equations by Ramirez, Mafe, Aguilella, et al. (2003) and Ramirez, Mafe, Alcarez, et al. (2003) for such membranes were compared to experimental results, and were able to qualitatively explain the observed permselectivity. Practical applications of such permselective membranes were investigated by Bluhm et al. (1999) studying cation transport through positively charged porous alumina membranes for the separation of radioactive and hazardous metal cations from contaminated water.

The first quantitative study of the EEE in a nanochannel was described by Plecis et al. (2005), who developed a simple model of the nanochannel permeability for varying ionic strength. The reference parameter for diffusion experiments is the permeability $P$, which is the constant that links the instantaneous flux $\phi$ to the concentration difference $\Delta c$,

$$
\phi=P \Delta c=\frac{S D}{d} \Delta c .
$$

The geometric permeability $P^{*}$ and the geometric cross section $S^{*}$ characterize the nanochannel when electrostatic interactions have a negligible effect on the trans-

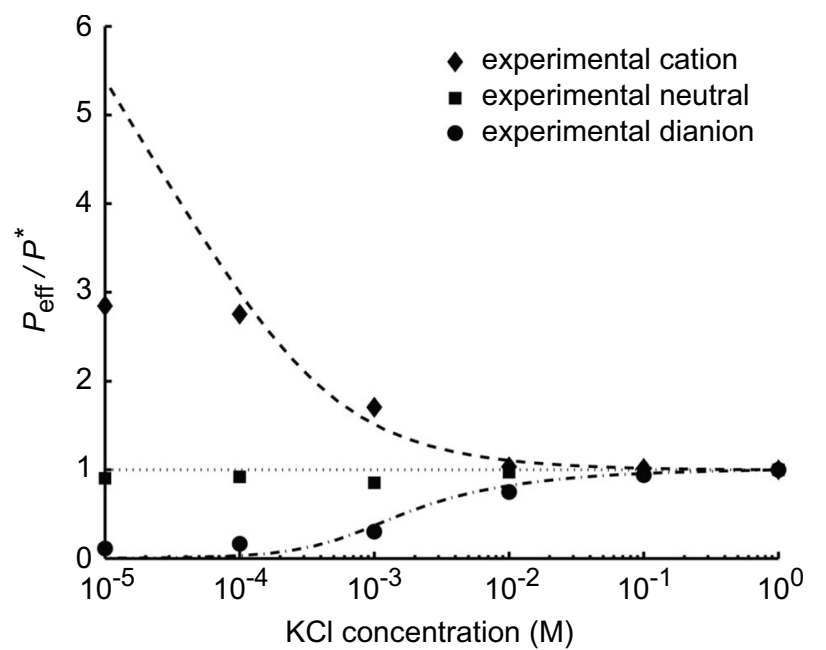

FIG. 13. Variation of the relative permeability $P_{\text {eff }} / P^{*}$ of a 50-nm-high nanochannel vs $\mathrm{KCl}$ concentration for three probe charges. Marks represent experimental measurements, whereas dotted lines are theoretical fits obtained with Eq. (53). Anions are excluded from the nanochannel when EDL overlap occurs at low $\mathrm{KCl}$ concentrations, in which regime cations are attracted, and therefore show enhanced transport through the nanochannel. Adapted from Plecis et al., 2005.

port rate (high ionic strength), whereas the effective permeability $P_{\text {eff }}$ and effective cross section $S_{\text {eff }}$ are used when the transport of charged species is affected by electrostatic forces.

When the exclusion and enrichment of ions occurs at low ionic strength, the local concentration of charged species is no longer homogeneous in the cross section of the nanochannel, because the EDL extension increases the counterion concentration near the charged surface. Therefore, the $z$ profile of the local concentration $c(x, z)$ (see Fig. 14) must be averaged to determine the effective concentration $c_{\text {eff }}(x)$ in the nanochannel in the presence of electrostatic forces. The ratio between $c_{\text {eff }}$ and $c^{*}(x)$ defines the exclusion-enrichment coefficient $\beta$, which is constant in the whole nanochannel and quantifies the EEE,

$$
\beta=\frac{c_{\mathrm{eff}}(x)}{c^{*}(x)}=\frac{\langle c(x, z)\rangle_{z}}{c^{*}(x)}=\frac{P_{\mathrm{eff}}}{P^{*}} .
$$

The exclusion-enrichment coefficient $\beta$ is higher than 1 in the case of enrichment, and lower than 1 in the case of exclusion. The measured relative permeability $P_{\text {eff }} / P^{*}$ for different charged probes is shown in Fig. 13, presenting an exclusion of anions and an enrichment of cations. No change in the permeability has been observed for the neutral probe. Qualitatively, these results are in accordance with the main hypothesis that EDL extension results in a higher permeability for counterions and a lower permeability for co-ions. However, the change in permeability is important when $2 \lambda_{D}<h$ before the classical EDL overlap, which means that electrostatic interactions between charged species and the walls are important prior to overlap. Moreover, the permeability is 

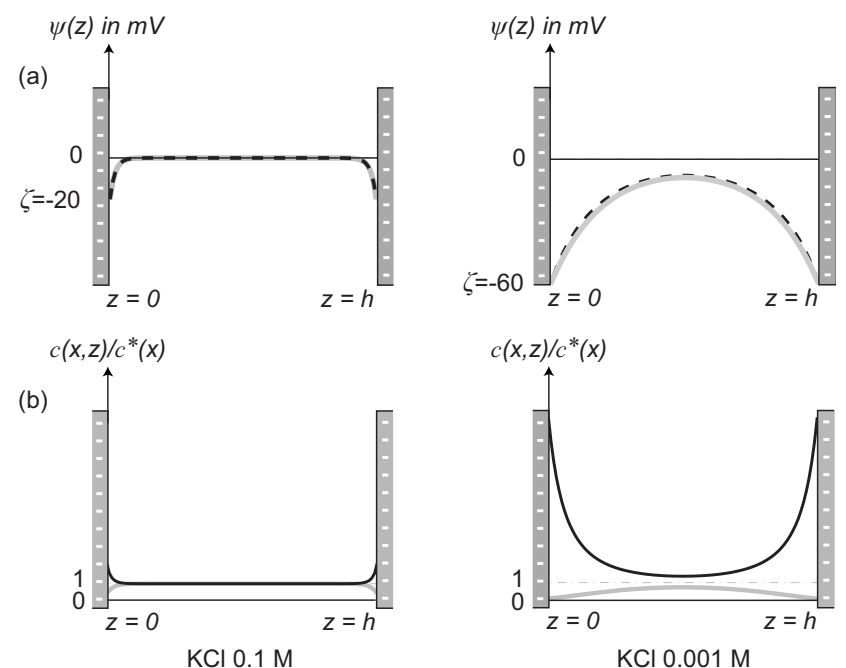

FIG. 14. Potential and concentration profiles in a 50-nm-high nanochannel for two $\mathrm{KCl}$ concentrations. (a) At a low ionic strength of $0.001 \mathrm{M} \mathrm{KCl}$, the potential remains negative in the nanochannel. The difference between the numerical solution of Eq. (6) (dotted black line) and the approximated analytical expression of Eq. (52) (gray solid line) is small. (b) Concentration profiles of charged species are determined by the Boltzmann equilibrium [Eq. (24)]. At low ionic strength, the enrichment of cationic species (black solid line, net charge $+1 e$ ) and the exclusion of anionic species (gray solid line, net charge -1 e) results. Adapted from Plecis et al., 2005.

not zero at low concentrations, which shows that EDL overlap does not signify complete anion impermeability of the nanochannel, as we might suppose from Fig. 12.

\section{Model of the exclusion-enrichment effect}

At low ionic strength, the EDL thickness becomes comparable to the nanochannel height, resulting in an overlap of the diffuse parts of the EDLs in the nanometer-sized aperture. It is useful to deduce the potential distribution in the case of EDL overlap in nanochannels, for which the exact potential is given by the DLVO theory (see Sec. II.A). A simplified solution for this theory is obtained by solving the Debye-Hückel approximation given in Eq. (7) with the appropriate boundary conditions, resulting in the following potential distribution (Hunter, 1981):

$$
\psi(z)=\frac{\zeta \cosh \left((h / 2-z) / \lambda_{D}\right)}{\cosh \left(h / 2 \lambda_{D}\right)} .
$$

The numerical solution of the complete PoissonBoltzmann equation [Eq. (6)] and the approximated potential presented in Eq. (52) are compared in Fig. 14(a) for different ionic strengths $(\mathrm{Qu}$ and $\mathrm{Li}, 2000$; Conlisk et al., 2002; Conlisk, 2005). It is known that the DebyeHückel approximation generally overestimates the electric potential. In order to achieve better precision, numerical solution of the Poisson-Boltzmann equation should be used. However, when the experimental uncertainty of permeability measurements is considered, the difference between the two predicted potentials remains small, and the Debye-Hückel approximation is usually sufficient.

Figure 14(b) shows that at a high ionic strength of $0.1 M \mathrm{KCl}$ where the potential is zero in most of the nanochannel, no significant change in the concentration is observed and $c_{\text {eff }}(x)$ is equal to $c^{*}$, except for a negligible region near the surface. At low ionic strength, where the electric potential remains high in the whole nanochannel, the exclusion of anionic species and enrichment of cationic probes becomes important. From Eqs. (51), (52), and (24), we obtain an analytical expression for the exclusion-enrichment coefficient,

$$
\beta=\frac{1}{h} \int_{0}^{h} \exp \left(\frac{-q \zeta}{k_{B} T} \frac{\cosh \left((h / 2-z) / \lambda_{D}\right)}{\cosh \left(h / 2 \lambda_{D}\right)}\right) d z .
$$

Equation (53) shows that the Debye length $\lambda_{D}$ and the characteristic dimension of the nanochannel $h$ are not the only parameters characterizing EEE, but the net charge $q$ of the diffusing species (see Sec. III.D) and the $\zeta$ potential of the surface are also important parameters for the quantification of this permselective effect. The $\zeta$ potential can be changed with surface charge modifications, and an inversion of the $\zeta$ potential is even possible with aluminum pretreatment (Horn and Onoda, 1978; Dilmore et al., 1979), resulting in an anion-selective nanochannel (Plecis et al., 2005).

\section{Partitioning at the interface}

The transport of large molecules through porous membranes was initially examined to understand the permeability of biological structures such as glomerular capillaries in the kidney, or walls of blood capillaries (Ferry, 1936; Pappenheimer et al., 1951; Renkin, 1954; Cevc, 1990). Later, renewed interest in fine pores occurred when track-etch processes allowed the fabrication of nanopores with better-controlled geometries (Bean et al., 1970), and models based on the hindered diffusion of macromolecules through pores had been developed (Beck and Schultz, 1970; Malone and Anderson, 1978). These models include two main sieving contributions: the exclusion of molecules from pores based on geometric effects and hydrodynamic drag on macromolecules due to the proximity of the pore walls. If molecules are comparable in size to the pore, hydrodynamic interactions between the molecules and pore walls are dependent on long-range intermolecular forces and steric restrictions. In the $1980 \mathrm{~s}$, progress was demonstrated in hindered transport through pores by the quantification of electrostatic interactions, the effects of solute concentration on partitioning and transport coefficients, and the influence of molecular configuration on transport rates (Smith and Deen, 1980; Deen, 1987).

Hereafter, we present a partitioning theory that accounts for size and electrostatic effects, and then we focus on electrostatic sieving. For the discussion of purely size-dependent sieving, we refer the reader to Sec. IV.B. 


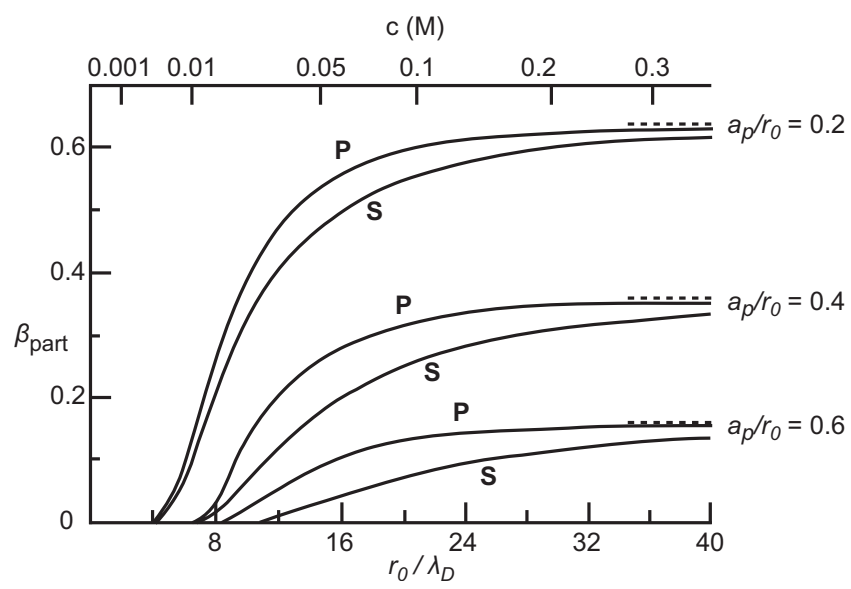

FIG. 15. Partition coefficient $\beta_{\text {part }}$ as a function of the solution concentration $c$, or of the ratio of pore radius $r_{0}$ to Debye length $\lambda_{D}$, for completely porous (P) and solid (S) spheres of identical size and total charge. Calculations were performed for $r_{0}=20 \mathrm{~nm}$, surface charge densities of walls and spheres of $0.01 \mathrm{C} \mathrm{m}^{-2}$, and a 1:1 univalent electrolyte. Dashed lines indicate that asymptotic values are approached at high ionic strength for each value of $a_{p} / r_{0}$, where $a_{p}$ is the particle radius. Adapted from Smith and Deen, 1983.

\section{Partition coefficient for colloids}

Smith and Deen (1983) theoretically studied electrostatic effects on the partitioning of spherical colloids between a dilute bulk solution and cylindrical pores. They defined the fundamental partition coefficient $\beta_{\text {part }}$, which expresses the ratio of the macromolecule concentration in the pore $c_{m}$ and the bulk $c_{m, b}$,

$$
\beta_{\text {part }}=\frac{c_{m}}{c_{m, b}}=2 \int_{0}^{1-a_{p} / r_{0}} \exp \left(\frac{\epsilon_{p}\left(r / r_{0}\right)}{k_{B} T}\right)\left(r / r_{0}\right) d\left(r / r_{0}\right),
$$

where $r$ is the radial coordinate and $\epsilon_{p}$ is the potential energy of interaction between the colloid and the pore wall. Note that the exclusion-enrichment coefficient $\beta$ assumes a point charge and accounts for electrostatic effects, compared to the partition coefficient $\beta_{\text {part }}$, which also includes size effects on partitioning. $\beta_{\text {part }}$ has been calculated by solving the linearized Poisson-Boltzmann equation and determining the free energy of the system and the interaction potential energy. In cases of solid or completely porous spheres and like-charged pores, the calculated partition coefficients are presented in Fig. 15. This demonstrates that completely porous spheres are electrostatically less hindered by their transport through a pore than solid spheres, because the elementary charge is distributed throughout the porous sphere, whereas for solid spheres the same number of charges reside on the sphere surface. With the increasing ratio of particle radius $a_{p}$ to pore radius $r_{0}$, the exclusion of colloids from like-charged pores becomes more pronounced.

\section{Electrostatic sieving of proteins}

In reverse osmosis, electrostatic partitioning is used to purify water by applying a pressure gradient over a nanoporous membrane, through which only pure water can pass; negatively charged molecules are rejected at the entrance of the cation-selective apertures (Smit, 1989; Hagmeyer and Gimbel, 1999). The rejection rate increases with dilution, resulting in high values at low ionic strength in nanochannels (Schoch, 2006). Electrostatic partitioning can be increased not only by dilution, but also by a higher molecule net charge $q$, because the exclusion-enrichment coefficient $\beta$ is exponentially dependent on $q$ [see Eq. (53)]. Therefore, electrostatic separation processes are favorable for biomolecules, as discussed subsequently, since these molecules typically bear a high net charge in their native state (Alexov, 2004).

The dominance of electrostatic interaction effects on the transport of proteins through a nanochannel has been demonstrated by Schoch et al. (2006). To understand their results, it is important to remember that proteins possess an isoelectric point $(p \mathrm{I})$ for a characteristic $p \mathrm{H}$ value at which they exhibit no net charge. Consequently, proteins are amphoteric molecules because they carry either positive $(p \mathrm{H}<p \mathrm{I})$, negative $(p \mathrm{H}>p \mathrm{I})$, or zero net charge $(p \mathrm{H}=p \mathrm{I})$. This fundamental behavior can be exploited by changing the $p \mathrm{H}$ value of the solution to separate proteins, because the net charge of a molecule affects its diffusion time through a nanochannel. Such $p \mathrm{H}$-switchable ion transport has been theoretically described for membranes (Jimbo et al., 2000; Ramirez, Mafe, Alcaraz, et al., 2003).

In order to demonstrate charge-based transport through a nanochannel, three lectin proteins (Foriers et al. , 1981) were investigated, which had similar molecular weights of $49 \mathrm{kDa}$ but different $p$ I values of 8.0, 8.2, and 8.8 (Schoch et al., 2006). For these three lectin proteins, the evolution of the apparent diffusion coefficient $D_{\text {app }}$ as the $p \mathrm{H}$ of the solution is varied between 6 and 11 is shown in Fig. 16. The curve illustrates three zones: for $p \mathrm{H} \leq 7$ and $p \mathrm{H} \geq 9$, the diffusion of lectin through the nanochannel is slow, whereas the diffusion is significantly faster for $p \mathrm{H}$ values between 7 and 9. Three peaks are visible in this part of the curve, one at $p \mathrm{H}$ $=7.5$, a second at $p \mathrm{H}=8.2$, and a third at $p \mathrm{H}=8.8$, and they can be attributed to the $p$ I values of the three proteins, showing a maximal diffusion coefficient through the nanochannel if they are neutral and do not have electrostatic interactions with channel walls (Burns and Zydney, 1999; Chun and Stroeve, 2002; Ku and Stroeve, 2004). For $p \mathrm{H} \geq 9$, all three proteins are negatively charged and excluded from the nanochannel according to the EEE [Eq. (53)]. Based on the exclusionenrichment effect, low diffusion times are expected for $p \mathrm{H} \leq 7$, when proteins are positively charged and consequently attracted by the nanochannel, but low apparent diffusion coefficients have been measured for this zone. To elucidate this discrepancy, the protein concentration in the nanochannel has been measured between $p \mathrm{H} 6$ 


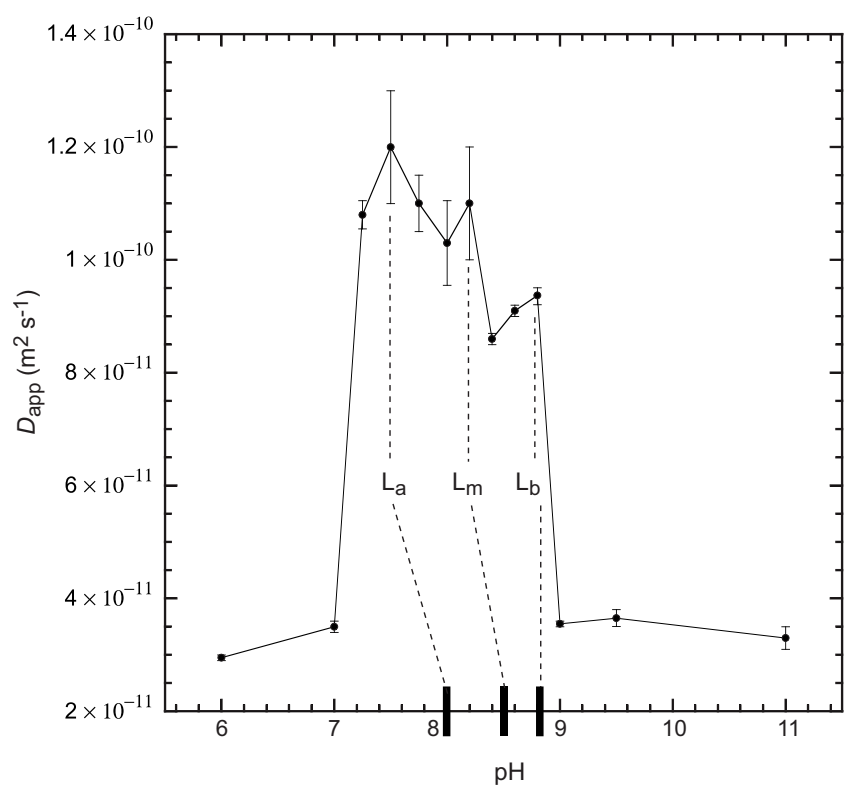

FIG. 16. Apparent diffusion coefficient $D_{\text {app }}$ of three lectin proteins as a function of the $p \mathrm{H}$ value of the solution, measured in a 50-nm-high nanochannel at an ionic strength of $10^{-3} M . D_{\text {app }}$ of the three lectin proteins shows three peaks, corresponding to the $p \mathrm{I}$ values of the acidic band lectin $L_{a}$, the middle band lectin $L_{m}$, and the basic band lectin $L_{b}$. The marks on the $p \mathrm{H}$ axis show the respective $p \mathrm{I}$ values of the lectins, which have been measured with isoelectric focusing. The connecting lines are for guidance only. From Schoch et al., 2006.

and 11 , showing maximum values for $p \mathrm{H} \leq 7$. Thus, it was concluded that proteins steadily enter the channel, but can hardly leave because they are retarded and reversibly adsorbed on the nanochannel walls (Gray, 2004; Lionello et al., 2005). The reversible adsorption of proteins to the surfaces also occurs between $p \mathrm{H} 7$ and 9, which leads to a slight $p \mathrm{H}$ change in the nanochannel because the local electrochemical potential near the protein regulates the ionizable surface group dissociation rather than the bulk chemical potential (Bostrom et al., 2005). These results confirm that adsorption and desorption kinetics become important in nanochannels (Drazer et al., 2002; Baldessari and Santiago, 2006).

(a) Considerations. Decreased diffusion coefficients of a protein for $p \mathrm{H}$ values below its $p \mathrm{I}$ were first measured in nanoporous membranes. $\mathrm{Ku}$ and Stroeve (2004) argued that at $p \mathrm{H}$ values below the $p \mathrm{I}$, the protein adsorbs on the nanopore surface. Additionally, the negative counterions of the positively charged protein are repelled from the negatively charged nanopores. This leads to the creation of a diffusion potential inside the nanopores that retards protein transport. Another explanation given by Burns and Zydney (1999) describes the energetic penalty associated with the distortion of the EDL around the charged protein by the channel wall. This distortion leads to net repulsive interactions, even when protein and pores have opposite charges. The third explanation is the charge regulation of biomolecules, which originates from the protein charge, which

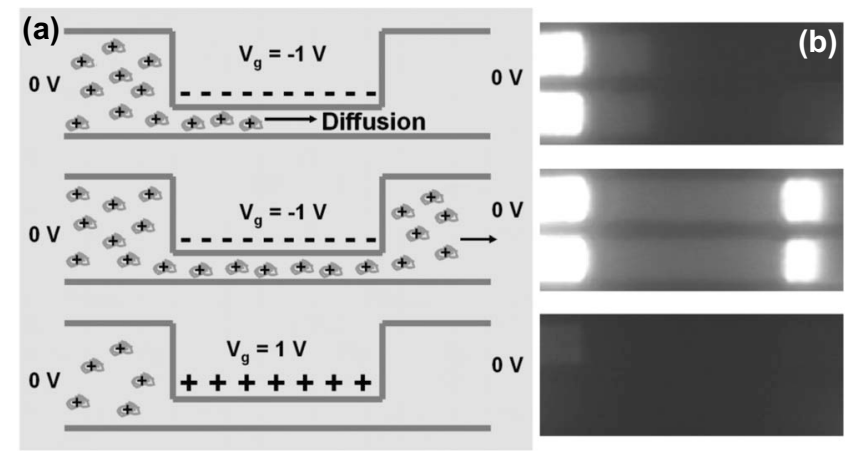

FIG. 17. Nanofluidic control of protein transport by a gate voltage without bias between the microchannels. When the gate voltage $V_{g}$ is negative, the positively charged protein avidin can diffuse through the nanochannel, whereas the protein is repelled when $V_{g}$ is positive. (a) Schematics and (b) corresponding fluorescence images. Adapted from Karnik, Castelino, and Majumdar, 2006.

is not constant but affected by nearby charged objects (Stahlberg and Jonsson, 1996; Pujar and Zydney, 1997; Zydney and Pujar, 1998; Menon and Zydney, 2000; Gohda and Pantelides, 2005; Lund et al., 2005). As a protein comes close to an oppositely charged stationary surface, the protein net charge will change because of the electric field from the surface.

(b) Protein adsorption. The adsorption of proteins from a solution to a surface is affected by many parameters (Norde, 1986; Nakanishi et al., 2001) and remains a controversial problem today. Polymers that resist protein adsorption are relevant for biomedical and bioanalytical applications. Poly(L-lysine) grafted with poly(ethylene glycol) (PLL-g-PEG) is a promising polymer to reduce nonspecific binding (Kenausis et al., 2000; Huang, Voros, De Paul, et al., 2002), and this has been verified in microfluidic devices (Marie et al., 2006).

\section{Active control of partitioning}

Partitioning can be controlled actively with field effects (Ghowsi and Gale, 1991; Schasfoort et al., 1999), which has been shown in nanochannels using external electrodes (Schoch and Renaud, 2005). Karnik, Fan, Yue, et al. (2005) and Karnik, Castelino, and Majumdar (2006) demonstrated that, similar to a field-effect transistor, a gate electrode on nanofluidic channels can be used to change their surface charge, enabling field-effect control as shown in Fig. 17. When the gate voltage $V_{g}$ is positive, only negatively charged molecules can diffuse across the nanochannels, and positively charged biomolecules are excluded. It has been proposed that such devices do not require EDL overlap (Stein et al., 2004; Daiguji et al., 2005). Unlike metal-coated nanoporous membranes, where the transport can be controlled by changing the potential applied to the membrane surface (Nishizawa et al., 1995; Chun et al., 2006), nanofluidic channels with a gate voltage enable researchers to address individual nanometer-sized openings. 


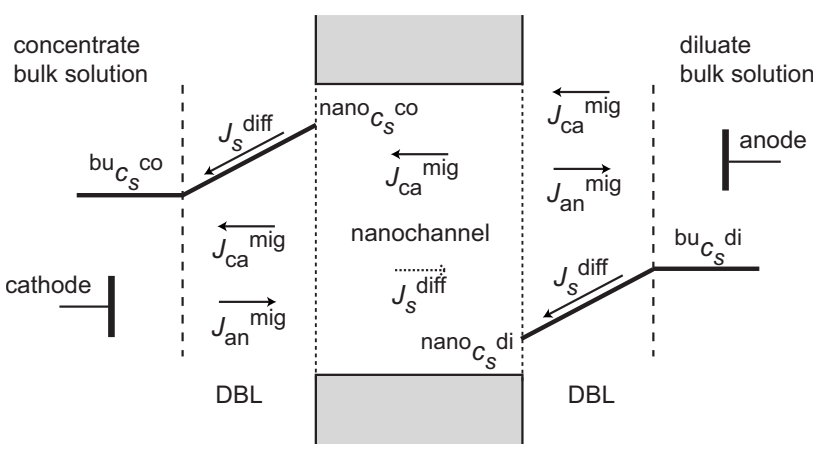

FIG. 18. Schematic illustrating concentration polarization at nanochannel interfaces. Concentration profiles of salt in the diffusion boundary layer (DBL) on both sides of the cationselective nanochannel are presented, as well as the fluxes per area $J$ of cations (ca), anions (an), and salt (s) in the DBL and in the nanochannel. The superscripts mig and diff refer to migration and diffusion, di and co refer to dilute and concentrate solutions, and bu and nano refer to bulk phase and nanochannel interface, respectively. Adapted from Strathmann, 2004.

Tunable ionic transport has been demonstrated by the application of a potential difference over a membrane (Schmuhl et al., 2004, 2005), which we investigate for nanochannels on a chip.

\section{E. Concentration polarization}

When an external electric field is applied through a nanochannel, electrokinetic transport is superimposed on diffusion. This ionic current transport can induce considerable forces on electrolytes in nanometer-sized apertures, as a result of which convection has to be considered (Helfferich, 1962). Concentration polarization does occur under these conditions, as well as a complex set of effects related to the formation of ionic concentration gradients in the electrolyte solution adjacent to an ion-selective interface. Such phenomena are complicated, and current electrokinetic theory (based on the equilibrium model of EDL) is inadequate to describe the complexity of the physics therein.

Subsequently, we describe fundamental aspects of concentration polarization and how these effects can be exploited for applications such as the preconcentration of molecules. A comprehensive review of nanopore ionic conductance in micrometer-scale analysis systems has recently been presented (Holtzel and Tallarek, 2007), and a detailed analysis of bulk concentration polarization for a two-parallel-plate electrode model with suddenly applied electric fields was provided by Bazant et al. (2004).

In solution, the electric current is transmitted by both anions and cations, and their transference number (see Sec. III.B.2) is about 0.5 for both ion types. However, in an ion-selective nanochannel, the current is mainly transported by counterions, resulting in a transference number close to 1 . At the anodic side of a cationselective nanochannel (see Fig. 18), the concentration of ions in solution is reduced because of the lower trans- port number of cations in the solution relative to that in the nanometer-sized opening (Strathmann, 2004; Nischang et al., 2006). Anions move in the opposite direction and cannot be compensated by the few anions that remain in the nanochannel. Because of electroneutrality, cations and anions are reduced by the same amount in front of the nanochannel on the anodic side, where a concentration gradient is established. At the other side of the nanochannel facing the cathodic side, cations accumulate because more cations are transferred through the nanochannel than are carried away by the electric current. The transference number of anions in the nanochannel is almost zero, resulting in their accumulation at the cathodic side of the nanometer-sized aperture (Petersen et al., 2004).

The concentration polarization is schematically presented in Fig. 18, which shows that cations are mainly transported by migrational flux $J_{\mathrm{ca}}^{\mathrm{mig}}$ through the nanometer-sized opening. Anions are largely excluded and their flux through the nanochannel is small compared to cation flux. The diffusional flux of salt $J_{s}^{\mathrm{diff}}$, including cations and anions, follows the gradient from the concentrate toward the dilute side. In the diffusion boundary layer (DBL) at both sides of the nanometersized aperture, cations and anions are transported by the migrational fluxes $J_{\mathrm{ca}}^{\mathrm{mig}}$ and $J_{\mathrm{an}}^{\mathrm{mig}}$, respectively, which are in opposing directions and thus generate a concentration gradient. This concentration gradient leads to a diffusional flux of salt $J_{s}^{\text {diff }}$, which is oriented toward the nanochannel on the dilute side and the bulk solution on the concentrated side of the nanochannel.

\section{Limiting current through nanochannels}

Concentration polarization can lead to a high salt concentration on the cathodic side of a nanochannel, and if it exceeds the solubility limits of the solution constituents, precipitation of salts may occur, resulting in an additional electric resistance. On the contrary, if the salt concentration is reduced to zero on the anodic side of the nanometer-sized opening, there are no ions available to carry the electric current. This results in a limited current $I_{\text {lim }}$, which corresponds to region II in Fig. 19. To calculate this limited current, the electrochemical analog to Fick's first law is considered [refer to Eq. (36)],

$$
I=n F A D \frac{\partial c}{\partial x} .
$$

The concentration gradient is commonly approximated by a linear extrapolation of the slope $c$ versus $x$ to $x=0$ (Rubinstein, 1995),

$$
I=n F A D \frac{c_{b}-c(x=0)}{\delta},
$$

where $c_{b}$ is the bulk concentration and $\delta$ is the DBL thickness present at solid-liquid interfaces (Levich, 1962; Cussler, 1997). For porous membranes, $\delta$ is typically between 10 and $400 \mu \mathrm{m}$ (Yaroshchuk et al., 2005). At the microchannel-nanochannel interface, it is assumed that 


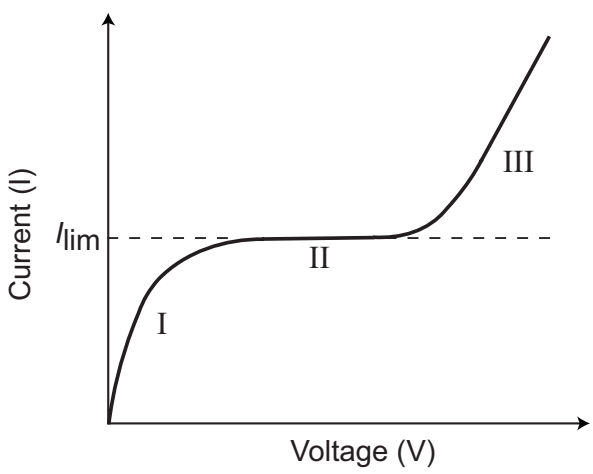

FIG. 19. Scheme of a typical voltage-current curve of a cationexchange membrane. In region $\mathrm{I}$, the current is increasing more or less linearly with the applied voltage according to Ohm's law. Due to concentration polarization, a limiting current is observed in region II with the magnitude $I_{\text {lim }}$. The classical theory of concentration polarization predicts a true saturation of the voltage-current curve (dashed line), but other effects lead to overlimited currents in region III. Adapted from Rubinstein and Zaltzman, 2000.

the dimension of the DBL is largely determined by the microchannel height (Rubinstein and Zaltzman, 2000; Kim et al., 2007). From Eq. (56), it can be seen that a maximal current is obtained when $c(x=0)=0$, resulting in the limiting current

$$
I_{\lim }=n F A D \frac{c_{b}}{\delta} .
$$

According to Eq. (57), which is based on the classical picture of concentration polarization, the limiting current cannot be exceeded unless ions other than salt ions become available for current transport in the saltdepleted DBL. This is the case when water dissociation leads to the production of $\mathrm{H}^{+}$and $\mathrm{OH}^{-}$ions, which will then carry the electric charges, resulting in so-called overlimited currents as in region III of Fig. 19. As a consequence, $p \mathrm{H}$ value shifts are obtained, with increasing $p \mathrm{H}$ values on the anodic side of the nanochannel and decreasing $p \mathrm{H}$ values on the cathodic side.

\section{Electroconvection for mixing}

The reason for transport in the overlimiting current regime III (see Fig. 19) is a subject of intensive discussion in the literature (Rubinstein, 1981; Rubinstein et al., 1997; Strathmann, 2004). It was concluded that, in addition to water dissociation, some mechanism of mixing must exist that is effective enough to destroy the DBL, because higher currents are possible if $\delta$ is reduced [see Eq. (57)]. This was confirmed by a straightforward experimental finding in which the surface of a cationexchange membrane was coated with a gel that does not allow mixing, and a saturation plateau was reached (dotted line in Fig. 19) (Maletzki et al., 1992; Rubinstein et al., 1997).

The mechanism for mixing was suggested by Dukhin (1991) to be electroconvection, which draws together the phenomena observed in all three regions of the voltage-

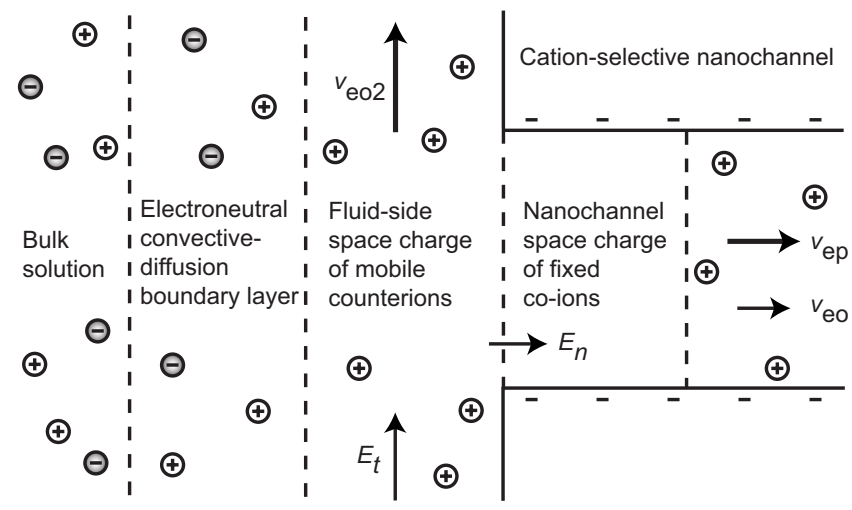

FIG. 20. Schematic of the anodic side of a cation-selective nanochannel under a high external electric field $E$. The normal field component to the bulk surface $E_{n}$ results in a nonequilibrium double layer of field-induced space charges that are mobile, and its counterparts are fixed co-ions inside the nanochannel. $E_{n}$ in combination with a tangential field $E_{t}$ result in an electro-osmotic velocity of the second kind $v_{\mathrm{eo} 2}$, which can destroy the DBL, leading to overlimited currents through the nanochannel. Electro-osmotic and electrophoretic velocities $v_{\text {eo }}$ and $v_{\text {ep }}$, respectively, are also shown. Adapted from Leinweber and Tallarek, 2004.

current curve. Two types of electroconvection are known: the classical electro-osmosis (the first kind, described in Sec. II.B.1) and electro-osmosis of the second kind (Dukhin, 1991; Mishchuk and Takhistov, 1995). The latter type results when tangential and normal electric fields are applied to a permselective interface leading to polarization of the double layer, a lateral pressure drop in the double layer, and a local inconsistency of the electroneutrality approximation. A nonequilibrium double layer of field-induced space charges is obtained at the opening of the permselective nanochannel (see Fig. 20), the thickness of which grows with increasing current density, and which can substantially exceed the Debye length. An applied potential over the nonequilibrium double layer $\Phi_{n}$ in combination with a tangential electric field to the bulk surface $E_{t}$ acts on these field-induced space charges, leading to an electro-osmotic velocity of the second kind,

$$
v_{\mathrm{eo} 2}=-\frac{\varepsilon_{0} \varepsilon_{r} E_{t}}{\eta} \Phi_{n}
$$

In contrast to electro-osmosis of the first kind, which is dependent on the $\zeta$ potential, $v_{\mathrm{eo} 2}$ is proportional to $\Phi_{n}$ and can therefore be much stronger. Electro-osmotic flow of the second kind results in convective instability and tends to destroy the DBL locally, thus overcoming diffusion limitation at the nanochannel interface and making overlimited currents possible. The DBL, therefore, transforms into a convective-diffusion boundary layer, consisting of an electroneutral part and a charged part (space-charge region) as drawn in Fig. 20. The counterpart of the mobile, field-induced space charges in the nonequilibrium double layer is balanced by an immobile region of field-induced space charges in the nanometer- 
sized aperture (Leinweber and Tallarek, 2004; Tallarek et al., 2005).

It is known from beds of ion-permselective particles (Leinweber and Tallarek, 2004; Tallarek et al., 2005), permselective membranes (Rubinstein and Zaltzman, 2000; Ben and Chang, 2002; Rubinshtein et al., 2002; Yaroshchuk et al., 2005), and permselective nanochannels (Bushong et al., 2007; Kim et al., 2007) that steadystate electroconvective eddies build up only slightly above the instability threshold, and at even higher field strengths, oscillations become chaotic and result in lowfrequency excess noise.

\section{Preconcentration of molecules}

We have described the ion enrichment and depletion that can be achieved on the cathodic and anodic sides of a permselective opening, respectively. These effects were first observed at the entrances of nanochannels by $\mathrm{Pu}$ et al. (2004), and represent functionalities unique to preconcentrate molecules on a chip. This concept is promising for $\mu \mathrm{TAS}$, because a challenge of miniaturized systems resides in the detection of highly diluted analytes in small volumes of solutions (Lichtenberg et al., 2002).

In Sec. III.D.2, we mentioned that co-ions are rejected at the entrance of counterion-selective nanochannels through which a pressure gradient is applied. This rejection leads to the preconcentration of co-ions, but not counterions. We emphasize that this is fundamentally different from the preconcentration mechanism using an electric field through nanochannels, resulting in the enrichment of both co-ions and counterions at the cathodic side of the nanometer-sized aperture. In addition to these charge-based preconcentration schemes, the enrichment of molecules at the entrance of apertures due to size exclusion has been demonstrated (Khandurina et al., 1999; Song et al., 2004; Foote et al., 2005; Hatch et al., 2006; Wang et al., 2007).

Here we focus on charge-based preconcentration using an electric field through a nanochannel, which is connected by two microchannels [see Fig. 21(a)]. Using this preconcentration scheme, it has been demonstrated that the preconcentration magnitude increases with the net charge of the analyte molecules and can therefore be used for biomolecules (Schoch, 2006). After $10 \mathrm{~min}$ of enrichment, preconcentration factors of 6.5 -fold and 18 fold have been measured for cations and dianions, respectively, and preconcentration factors greater than 600-fold have been achieved for recombinant green fluorescent protein (rGFP) [see Fig. 21(b)]. These measurements were performed with a 50-nm-high nanochannel in phosphate buffer at an ionic strength of $10^{-4} \mathrm{M}$ and a $p \mathrm{H}$ value of 7.5. The $p \mathrm{I}$ of rGFP is 4.5 , and, as a result, this protein has a high net charge in the experimental buffer. Hence, the protein experiences a strong repulsive force from the like-charged nanochannel, resulting in efficient preconcentration. Figure 21(b) shows that the preconcentration factor increases with time and increasing magnitude of the applied voltage, indicating that
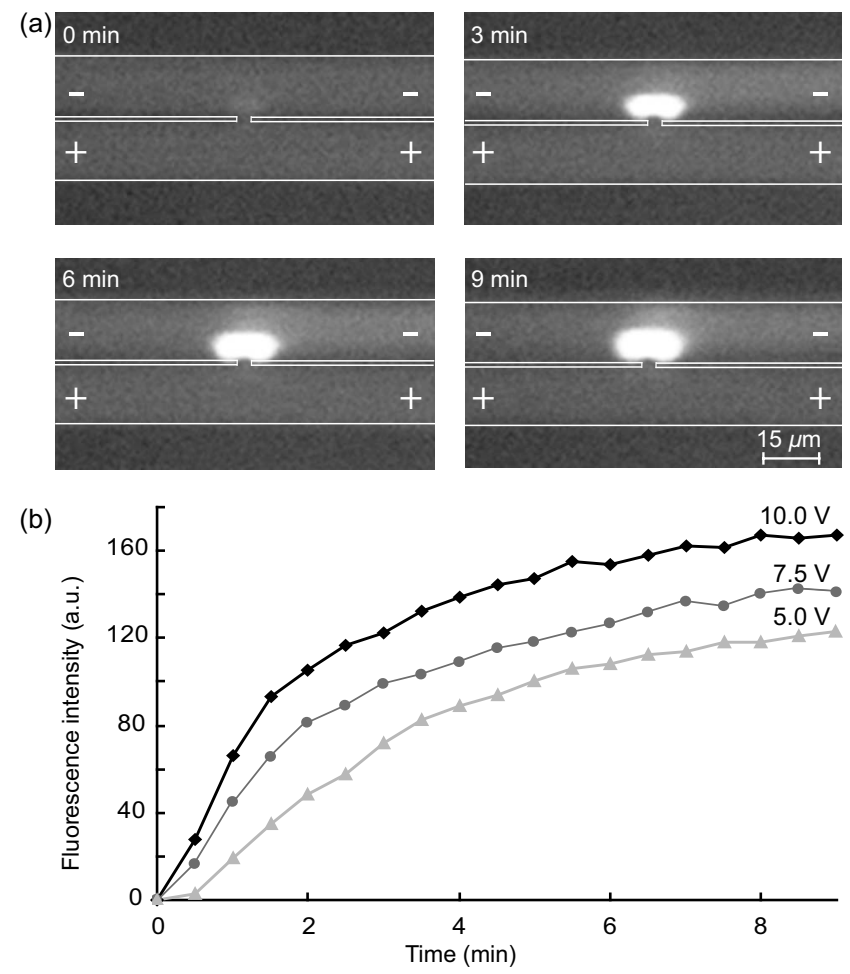

FIG. 21. Preconcentration of proteins in the cathodic microchannel in front of a permselective nanochannel. (a) Fluorescence images of preconcentrated recombinant green fluorescent protein (rGFP) inside the upper microchannel show that the preconcentration zone increases in size and concentration over time (illustrated for $0,3,6$, and $9 \mathrm{~min}$ at $10 \mathrm{~V}$ ). The size of the depleted region is typically much larger than the enrichment zone, and hence cannot be captured in these images. (b) Fluorescence intensity of preconcentrated rGFP as a function of time for three values of the applied voltage. The linear behavior between the protein concentration and the measured fluorescence intensity (not shown) has been used to calculate a preconcentration factor greater than 600-fold after 9 min at 10 V. Adapted from Schoch, 2006.

concentration polarization is a dynamic positive feedback process. A saturating value is obtained after several minutes, which can be caused by several mechanisms, and will require further investigation to understand fully. Numerical solutions of the concentration polarization in porous media (Leinweber et al., 2005) and membranes (Bath et al., 2000; Bhattacharjee et al., 2001) have been performed and could provide further insight when adapted to nanochannels.

The highest preconcentration factors of a millionfold were reported by Wang, Stevens, and Han (2005), who used electrokinetic trapping in combination with electro-osmotic flow of the second kind to preconcentrate analyte molecules on the anodic side of a chargeselective nanochannel. As described for concentration polarization, an electric field through a permselective nanochannel creates a field-induced depletion zone on the anodic side of the nanometer-sized opening, and this depletion region represents an energy barrier and prevents charged biomolecules from entering that zone. Thus, biomolecules get concentrated in the microchan- 
nel at the interface to the depletion region. A second electric field gradient has been applied along the anodic microchannel, which leads to an electro-osmotic flow of the second kind in this channel, and hence supplies the preconcentration zone efficiently with biomolecules. This electrokinetic trapping and collection procedure can be run for several hours, allowing high preconcentration factors.

\section{MACROMOLECULE SEPARATION MECHANISMS USING NANOMETER-SIZED STRUCTURES}

During the past several years, significant interest has emerged in separating macromolecules, and it has been demonstrated that nanofluidics can accomplish this task by exploiting various mechanisms. In this section, we report the physical principles of separation mechanisms using nanometer-sized structures on a chip, which represents a growing field with potential applications for $\mu$ TAS (He et al., 1998; Eijkel and van den Berg, 2006; Haber, 2006). Such microfabricated regular sieving structures hold promise as an alternative to gels or capillaries to improve biomolecule separation speed and resolution. Until recently, the main focus has been placed on the separation of long DNA molecules (Chou et al., 2000) due to the Human Genome Project (Craig Venter et al., 2001; Lander et al., 2001), limitations in gel electrophoresis (Turmel et al., 1990; Gygi et al., 2000; Lambert et al., 2005), and well-known DNA separation physics (Giddings, 1965, 1991; de Gennes, 1979; Stellwagen et al., 1997; Righetti et al., 2002; Slater et al., 2003). The interest in separation techniques for smaller physiologically relevant biomolecules is increasing (Corthals et al., 1997; Anderson and Anderson, 1998; Rabilloud, 2002; Freire and Wheeler, 2006).

\section{A. Entropic trapping}

When a DNA molecule is in a relaxed state and in equilibrium, it has a spherical shape with a radius of gyration $R_{0}$. However, if the molecule is forced into an opening with dimensions smaller than $2 R_{0}$, it has to deform from its state of minimal energy. The entry of the DNA strand into such a constricted space is called entropic trapping (Muthukumar and Baumgartner, 1989; Smisek and Hoagland, 1990; Rousseau et al., 1997), and the entropic trapping effect of polymer chains in microfabricated structures has been directly observed (Han and Craighead, 1999, 2000; Han et al., 1999).

Han and Craighead (2000) used a nanofilter column consisting of alternating deep regions $\left(t_{d} \approx 2 \mu \mathrm{m}\right)$ and shallow constrictions $\left(t_{s} \approx 90 \mathrm{~nm}\right)$, over which an electric field was applied [see Fig. 22(a)]. It has been demonstrated that longer DNA molecules move faster through the column because they have a better chance of escaping entropic traps. This is due to the larger contact area of the DNA molecule with the thin region [Fig. 22(b)], which results in a shorter entropic trapping lifetime. An important parameter describing the trapping lifetime is the magnitude of the electric field in the nanochannel
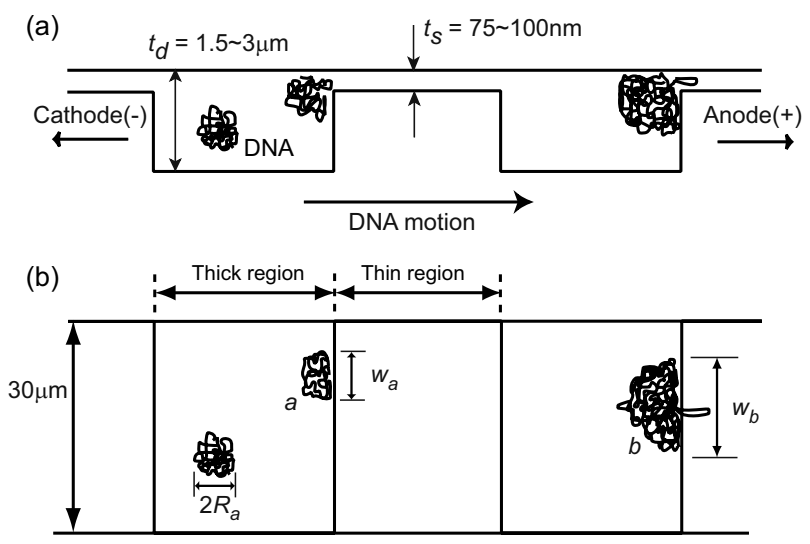

(c)

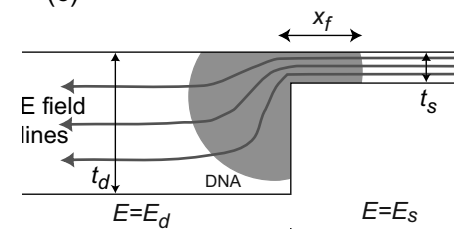

(d)

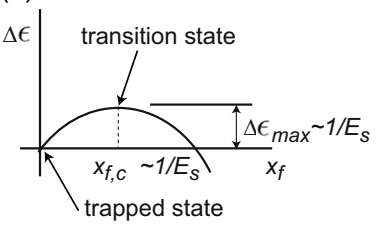

FIG. 22. Entropic trapping of DNA in a nanofilter column. (a) Cross-sectional schematic of the device consisting of alternating shallow $\left(t_{s}\right)$ and deep $\left(t_{d}\right)$ regions. Electrophoresed DNA molecules are trapped whenever they meet a thin region. (b) Top view of the device. The width $w$ of the DNA molecule in contact with the thin region determines the trapping lifetime, and therefore the overall mobility. $R_{0}$ is the radius of gyration. From Han and Craighead, 2000. (c) Escape of a DNA molecule from the trap, where $x_{f}$ is the length of the DNA strand in the filter. (d) Energy $\Delta \epsilon$ vs $x_{f}$. The transition state at which the strands overcome the energy barrier is when $x_{f}=x_{f, c}$. Adapted from Han et al., 1999.

$E_{s}$, because the necessary energy to overcome an entropic trap $\Delta \epsilon_{\max }$ is proportional to $1 / E_{s}$ [Fig. 22(d)]. The trapping lifetime $\tau$ for intermediate electric fields $\left(\Delta \epsilon_{\max } \approx k_{B} T\right)$ is

$$
\tau=\tau_{0} \exp \left(\Delta \epsilon_{\max } / k_{B} T\right)=\tau_{0} \exp \left(\alpha / E_{s} k_{B} T\right),
$$

where $\tau_{0}$ is the trapping lifetime at high electric fields.

Han and Craighead (2002) optimized all parameters affecting the separation of long DNA molecules across entropic traps. The outcome of this study was that even mega-base-pair DNA strands can be separated about ten times faster with entropic traps than with conventional pulsed field gel electrophoresis. Simulations of the trapping lifetime as a function of molecular size and electric field strength have been presented by Tessier and Slater (2002) and Tessier et al. (2002).

\section{B. Ogston sieving}

Ogston sieving (Ogston, 1958; Rodbard and Chrambach, 1970) is fundamental for describing the mobility of globular molecules in gel electrophoresis, which is widely used to separate molecules by size (Giddings et al., 1968; de Gennes, 1971; Semenov et al., 1995; Viovy, 2000; Slater et al., 2002). The sieving mechanism is described by a partitioning process (see Sec. III.D) due to 
steric repulsion, and the partition coefficient in gels is therefore represented by the volume fraction of the gel that is available to the molecule (free volume). In a nanofilter column, it has been demonstrated that separation is obtained due to Ogston sieving if a molecule is smaller than the nanoconstriction (Fu et al., 2005), and hence does not need to deform, as observed in entropic trapping. These authors have explained Ogston sieving in a nanofilter column by the limited configurational freedom of a molecule inside a constriction zone due to steric repulsions from the walls, creating a sizedependent configurational energy barrier at the nanoconstriction entrance.

Ogston sieving of rodlike DNA molecules has been achieved in nanofilter columns (Fu et al., 2005), selfassembled colloidal arrays (Zeng and Harrison, 2007), and nanopillar arrays with moderate electric fields (Kaji et al., 2004). In contrast to long DNA molecules which separate according to entropic trapping, the overall mobility of rodlike molecules in a nanofilter column decreases with increasing length. Fu et al. (2006) calculated the mean trapping time $\tau$, based on the equilibrium partitioning and Kramer's theory,

$$
\tau=\frac{\alpha N}{E^{2} \beta_{\mathrm{part}}} \exp \left(-\frac{N q_{\mathrm{DNA}}^{*} E t_{d}}{k_{B} T}\right)
$$

where $N$ is the DNA base pair number and $q_{\mathrm{DNA}}^{*}$ is the effective DNA charge per base pair. These authors have shown that experimental evidence exists of the crossover from Ogston sieving to entropic trapping at around $2 R_{0} / t_{s} \approx 1$, which is at 1000-2000 base pairs in their device with $t_{s}=73 \mathrm{~nm}$.

Chromatographic separations are usually performed at near equilibrium (Giddings et al., 1968), and Nabil et al. (2007) calculated that enhanced separations through nanofilter columns could be obtained with high-field electrophoresis. Under strong electric fields, molecules are convected from shallow constriction to shallow constriction without diffusing into the deep region, and they must be aligned in the flow direction to avoid interactions with the walls. Rotational diffusion is higher for short DNA molecules because longer (but still rodlike) DNA molecules can conserve their orientation from the previous trap with a higher probability, and therefore they elute first via torque-assisted escape.

\section{Entropic recoil}

When DNA molecules are driven into a high-entropy region from a zone of lower configurational entropy, they experience a confinement-induced entropic force. A pulsed electric field has been used to partially insert DNA molecules into a nanopillar array, and after removal of the electric field they relax into their natural state (Turner et al., 2002). During the pulse period, shorter DNA molecules get fully inserted into the nanopillar array, while longer DNA molecules are still straggling at the interface from low to high entropy and are observed to recoil entirely out of the confinement zone

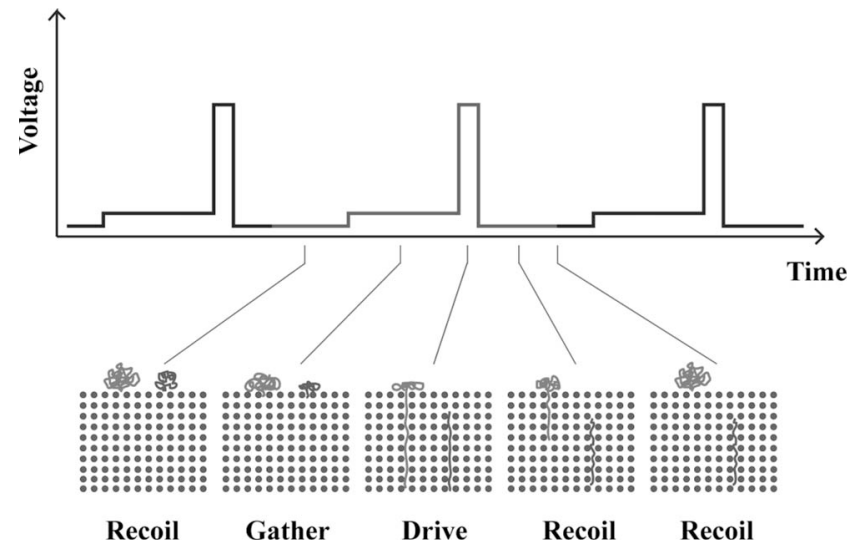

FIG. 23. Scheme for separation of DNA strands at the interface with a nanopillar array. During the gather phase, molecules are accumulated at the interface with the pillar region. Then, a pulse (drive) inserts shorter molecules fully, but longer molecules only partially, in the constriction zone. During the recoil phase, longer strands backtrack and remain in the pillarfree region. From Cabodi, Turner, and Craighead, 2002.

(Fig. 23). The ratio between the length $x_{p}$ of the DNA strand inserted into the pillar array and the entropic force $F_{e}$ has been calculated as (Turner et al., 2002),

$$
x_{p}=\sqrt{-\frac{2 F_{e}\left(t-t_{0}\right)}{f_{s}}},
$$

where $t_{0}$ is the time at which the molecule completes its recoil and $f_{s}$ is the specific friction coefficient. The entropic force $F_{e}$ has been investigated by Mannion et al. (2006). Equation (61) shows that the entropic force, which acts on the molecule during the recoil phase, is independent of the DNA length. Therefore, separation of DNA molecules can be obtained with pulsed electric fields, and, by progressively increase in the voltage pulses, shorter molecules are progressively separated from the longer ones (Cabodi, Turner, and Craighead, 2002).

\section{Anisotropy for continuous-flow separation}

The continuous-flow operation of a separation device permits continuous and convenient harvesting of purified biomolecules into specific reservoirs, allowing for further analysis of the biomolecules of interest. This represents a more favorable separation scheme than systems in which an analyte gets separated into its components but is finally eluted into the same reservoir. Continuous-flow separation has been demonstrated using micrometer-sized structures based on the sieving mechanisms of DNA length-dependent backtracking (Huang, Tegenfeld, Kraeft, et al., 2002), rectification of Brownian motion (Chou et al., 1999; Austin et al., 2002; Cabodi, Chen, Turner, et al., 2002; Huang, Silberzan, Tegenfeldt, et al., 2002) based on ratchets [proposed by Duke and Austin (1998) and Ertas (1998)], and asymmetric bifurcation of laminar flow (Huang et al., 2004). 


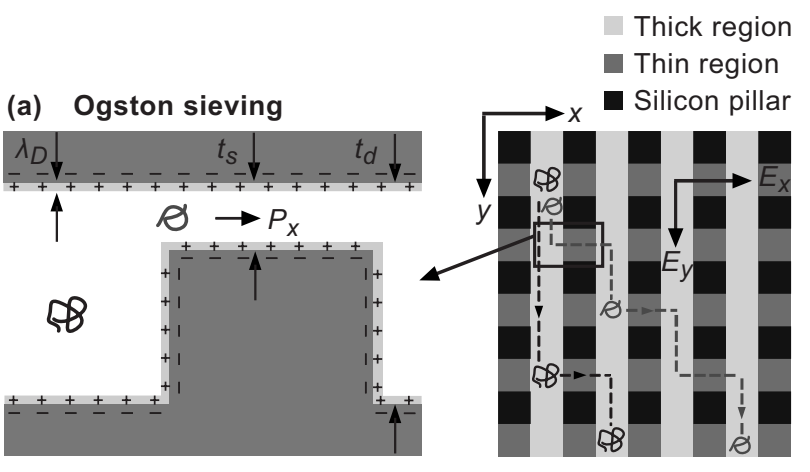

(b) Entropic trapping

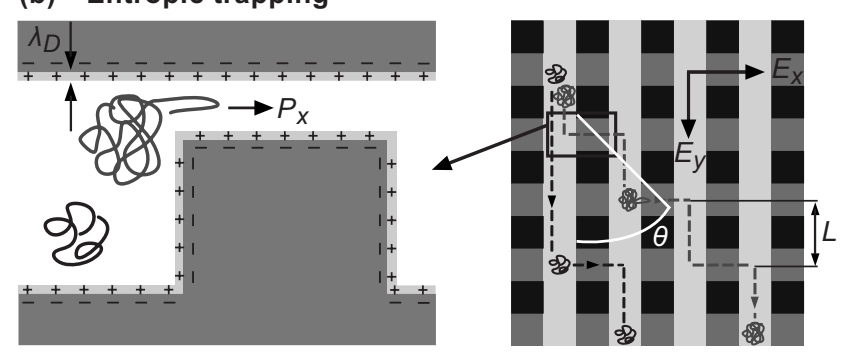

(c) Electrostatic sieving

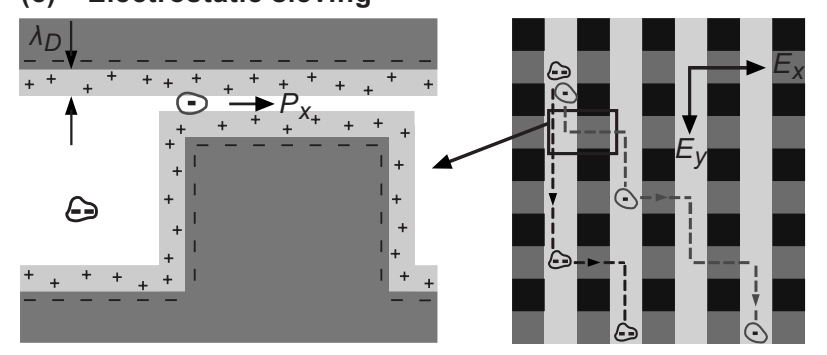

FIG. 24. Bidirectional motion of negatively charged macromolecules in an anisotropic nanofilter array, driven by two orthogonal electric fields $E_{x}$ and $E_{y}$. (a) Ogston sieving and (b) entropic trapping are obtained when $\lambda_{D} \ll t_{s}$. In this regime at high ionic strength, steric interactions dictate the jump dynamics and the passage rate $P_{x}$. (c) Electrostatic sieving becomes dominant when $\lambda_{D} \approx t_{s}$. The mean drift distance $L$ plays a determinant role for the migration trajectory, with a shorter $L$ leading to a larger stream deflection angle $\theta$, where $\theta$ is defined with respect to the positive $y$ axis. Silicon pillars serve as supporting structures to prevent collapse of the top ceiling. Adapted from Fu et al., 2007.

We have discussed mechanisms that can separate large macromolecules, and their applicability to smaller, physiologically relevant macromolecules remains questionable (Austin et al., 2002; Huang, Silberzan, Tegenfeldt, et al., 2002). To overcome this limitation, the properties of an anisotropic nanofluidic array have been exploited by Fu et al. (2007) to separate short DNA and proteins under native conditions in continuous flow. The anisotropy relies on different sieving characteristics along two orthogonal directions $x$ and $y$ within the array, where only deep channels are designed in the $y$ direction, while the $x$ direction consists of alternating deep channels and shallow nanofluidic constrictions (see Fig. 24). Two orthogonal electric fields $E_{x}$ and $E_{y}$ are used to drive the molecules through the channels.
This unique molecular sieving structure has demonstrated continuous-flow separation of DNA and proteins based on either size or charge (Fu et al., 2007). The shallow nanofluidic channels represent energy barriers to both steric and electrostatic interactions between charged biomolecules and charged nanofilter walls, resulting in the sieving mechanisms of Ogston sieving (see Sec. IV.B), entropic trapping (see Sec. IV.A), or electrostatic sieving (see Sec. III.D.2). Size-based separations due to Ogston sieving and entropic trapping are performed at high ionic strength, where the Debye length $\lambda_{D}$ is negligible compared to the nanofilter shallow region $t_{s}$, whereas low-ionic-strength solutions, when $\lambda_{D}$ becomes comparable to $t_{s}$, are used for electrostatic sieving. The separation of differently sized or charged biomolecules is obtained because macromolecules have different mean characteristic drift distances $L$ in the deep channels between two consecutive nanofilter crossings, leading to distinct stream deflection angles $\theta$ and jump passage rates $P_{x}$ (see Fig. 24).

\section{NANOPORES AND NANOWIRES FOR LABEL-FREE BIOMOLECULE DETECTION}

There are two main types of detection methods that are commonly integrated into $\mu$ TAS: fluorescent and electrical detections. Optical approaches are straightforward and provide direct visual proof. But with regard to industrial applications, a lab-on-a-chip device should be inexpensive, and electronic sensors are therefore favorable. Additionally, electrical characterizations do not require fluorescent dyes, which can cause variations in the net charge, conformational changes, or steric hindrance of the analyte (Richards et al., 1999; Ramachandran et al., 2005). In this section, we present promising electrical and label-free biomolecule detection methods, which are based on nanofluidic objects such as nanopores and nanowires.

\section{A. Biomolecule translocations through nanopores}

Kasianowicz et al. (1996) demonstrated that a DNA molecule can be detected as a transient decrease in the ionic current when it passes through a nanopore, and the passage duration allows for the determination of polymer length. If each base in the DNA molecule were to modulate the signal in a specific and measurable way during translocation, then the base sequence could be determined at rates between 1000 and 10000 bases per second (Deamer and Akeson, 2000). Thus, the ultimate goal of this technique is to realize rapid DNA sequencing (Howorka et al., 2001; Wang and Branton, 2001; Meller, 2003; Ashkenasy et al., 2005; Chan, 2005).

It has been proposed that the required measurement sensitivity to distinguish individual bases might be achievable with embedded electrodes in the walls along the axis of a synthetic nanopore (Lagerqvist et al., 2006). However, the transverse conductance across DNA fragments between electrodes depends critically on geomet- 
ric rather than electronic structural properties (Zhang et al., 2006), and other measurement techniques have been realized with embedded electrodes in the nanopores. We further present interesting effects of biological and synthetic nanopores, which have been observed on the way to electrical DNA sequencing.

\section{Biological nanopores}

For nanopore sequencing, an opening at the nanometer scale is required, and $\alpha$-hemolysin has been chosen for such investigations (Bayley and Cremer, 2001; Meller et al., 2001; Vercoutere et al., 2001; Vercoutere and Akeson, 2002). This transmembrane $33 \mathrm{kD}$ protein is isolated from Staphylococcus aureus; it has an asymmetric structure and a diameter of approximately $1.4 \mathrm{~nm}$ at its narrowest point. Ion channels switch their conformation between open and closed states, which is called ion channel gating (Bezrukov and Winterhalter, 2000; Siwy and Fulinski, 2002b; Beckstein et al., 2003; Sasaki et al., 2006; Schmidt et al., 2006). However, at neutral $p \mathrm{H}$ and high ionic strength, $\alpha$-hemolysin remains open, unlike most membrane channels, and allows a steady ionic current of $\sim 100 \mathrm{pA}$ to pass at an applied voltage of $100 \mathrm{mV}$ (Kasianowicz et al., 1996). The electric potential distribution along the length of the pore was estimated by Howorka and Bayley (2002).

The model system $\alpha$-hemolysin has allowed determining the length of single-stranded DNA and RNA, which is proportional to the measured translocation duration (Kasianowicz et al., 1996). Other important parameters for the characterization of translocation events are the measured current amplitude and the characteristic dispersion of values for individual translocation durations, which have also been used to discriminate different types of polynucleotides of similar length (Meller et al., 2000) or to probe DNA-protein complexes (Hornblower et al., 2007). Coarse-grained descriptions of polymer translocations have been developed (Lubensky and Nelson, 1999; Muthukumar and Kong, 2006), and an active control technique to study the dynamics of singlemolecule events has been established (Bates et al., 2003). Furthermore, single-molecule electrophoresis allows for the recognition of a single base in an individual DNA strand (Ashkenasy et al., 2005), the degree of phosphorylation and contamination (Wang et al., 2004), specific sequences (Howorka et al., 2001), and unzipping of individual DNA hairpin molecules (Mathe et al., 2004).

\section{Synthetic nanopores}

Despite these promising results, the biological nanopore $\alpha$-hemolysin cannot be redesigned at will, and it has a lower stability with buffer $p \mathrm{H}$ and ionic strength than a solid-state nanopore (Dekker, 2007). Thus, a broader range of molecules with a wider variety of conditions $(p \mathrm{H}$, ionic strength, temperature, functionalized surface) can potentially be investigated with a fabricated nanopore in an insulating material.

We show in Sec. VI.F how synthetic cylindrical and conical nanopores can be fabricated. These pores have
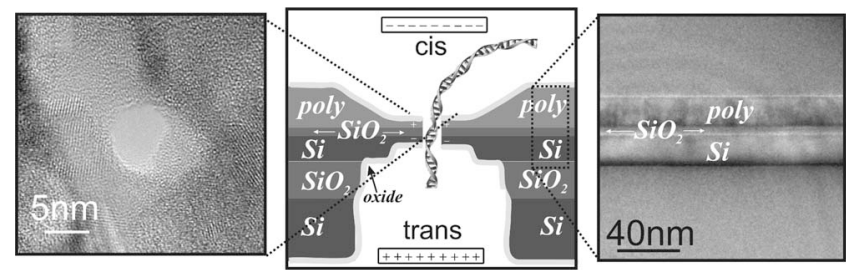

FIG. 25. Nanopore in a metal-oxide-semiconductor capacitor membrane, in which the biosensor was simulated to have single nucleotide resolution. Left panel: Image (top view) of a nanopore sputtered into the capacitor membrane. Center panel: Schematic cross section of the novel biosensor. Right panel: Cross-sectional transmission electron microscope image of the capacitor membrane. From Gracheva et al., 2006.

been used to investigate the translocation of DNA in folded and unfolded states (Li et al., 2003; Chen, Gu, Brandin, et al., 2004; Fologea, Gershow, Ledden, et al., 2005; Storm, Chen, Zandbergen, et al., 2005), the translocation duration of DNA (Heng et al., 2004; Mara et al., 2004; Karhanek et al., 2005; Harrell et al., 2006), which allows unraveling channel-molecule interactions with single-base-mismatch resolution (Iqbal et al., 2007), the blocked current amplitude during translocation of proteins (Han, Schurmann, Mondin, et al., 2006), and nanobubbles, which dominate the noise (Smeets, Keyser, $\mathrm{Wu}$, et al., 2006). Zhao et al. (2007) revealed recently that the voltage threshold for permeation of double-stranded DNA bound to a restriction enzyme depends on the genetic sequence. Additionally, it has been reported that, at low electric fields, double-stranded DNA cannot permeate a pore smaller than $3 \mathrm{~nm}$, while single-stranded DNA can, due to the difference in the DNA diameter. At higher threshold voltages, however, double-stranded DNA can be forced through (Heng et al., 2006). This threshold leads to the stretching of DNA in the pore (Heng et al., 2005) as a function of the buffer $p \mathrm{H}$. Interestingly, the translocation time is not linearly related to DNA length, but grows as a power of the polymer length, which could be due to the hydrodynamic drag of the DNA polymer section outside the pore (Fologea, Uplinger, Thomas, et al., 2005; Storm, Storm, Chen, et al., 2005). Calculations of polymer translocations (Muthukumar, 2001, 2003; Slonkina and Kolomeisky, 2003; Ghosal, 2007) and simulations of the sizing (Heng et al., 2004) and microscopic kinetics (Lubensky and Nelson, 1999; Aksimentiev et al., 2004) of DNA during passage through a synthetic nanopore have been performed.

Instead of measuring the blockade current with a typical two-terminal measurement, Gracheva et al. (2006) demonstrated voltage sensing of DNA with two additional electrodes in a metal-oxide-semiconductor capacitor membrane in which the nanopore resides (Fig. 25). This promising four-terminal measurement configuration enables electrical DNA molecule sequencing because the induced voltage signal of the translocating DNA on the electrodes in the membrane is characteristic of the electronic structure of the DNA. 


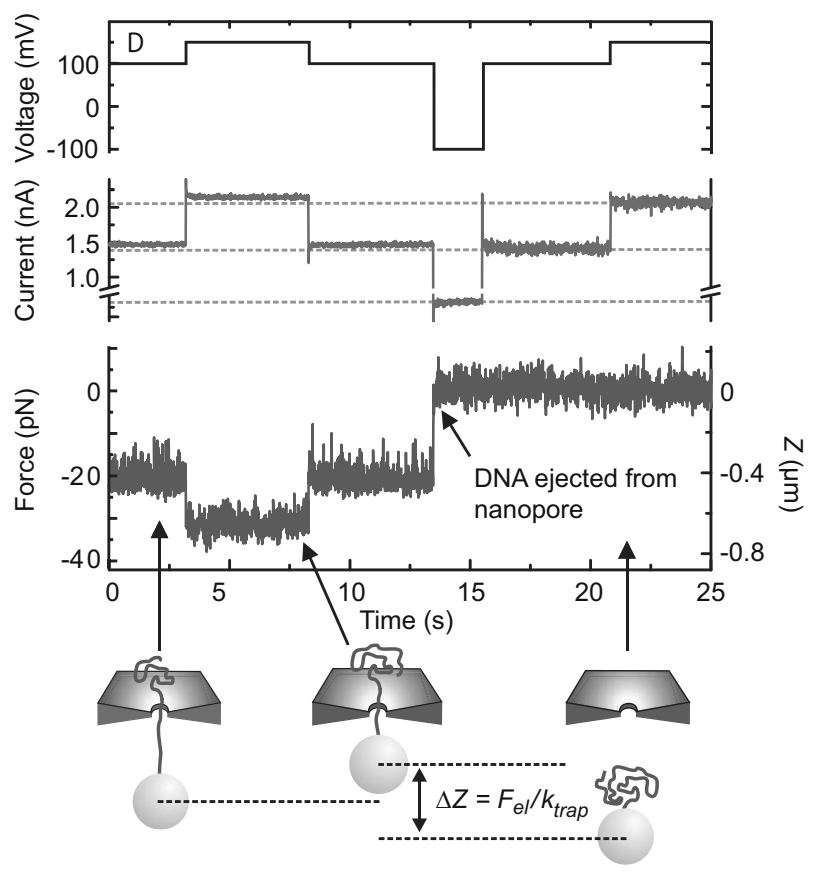

FIG. 26. Simultaneous current and force measurements of a DNA molecule in a nanopore at $0.1 \mathrm{M} \mathrm{KCl}$. Differences in the applied voltage change the bead position until the electrical force $F_{\mathrm{el}}$ is equal to the optical force $F_{\mathrm{ot}}$. Reversing the voltage at $t=13 \mathrm{~s}$ drives the DNA strand out of the pore, returning the open-pore current (dashed lines). The nanopore axis is defined in the $z$ direction, and $k_{\text {trap }}$ is the trap stiffness. From Keyser, Koeleman, Van Dorp, et al., 2006.

In order to understand the physics of the translocation process, the forces acting on the DNA have to be investigated. By combining ionic current measurements with optical tweezers, Keyser, Koeleman, Van Dorp, et al. (2006) determined the electrical force acting on a single DNA molecule in a solid-state nanopore. In their experimental configuration, the end of a DNA molecule is attached to a bead, which is optically trapped with an infrared laser in close proximity to the nanopore opening (Keyser, van der Does, Dekker, et al., 2006). Voltage application drives the DNA strand into the nanopore, and an electrical force $F_{\text {el }}$ is exerted on the bead, changing its position in the optical trap until the optical force $F_{\mathrm{ot}}$ is balanced by $F_{\mathrm{el}}$. When the applied voltage $V_{a}$ is changed (see Fig. 26), the force can be measured according to $F_{\mathrm{el}}=q_{\mathrm{DNA}}^{*} \Delta V_{a} / l_{b}$, where $l_{b}$ is the distance between two base pairs. The force acting on a single DNA molecule is $0.24 \mathrm{pN} \mathrm{mV}^{-1}$, which is independent of salt concentration from $0.02 M$ to $1 M \mathrm{KCl}$. This leads to an effective charge of $q_{\mathrm{DNA}}^{*}=0.5$ electron per base pair (bp), corresponding to a $75 \%$ reduction of the bare DNA charge. This value is similar to $0.48 e \mathrm{bp}^{-1}$, as predicted by Manning (1978) due to electrostatic counterion condensation. However, for DNA molecules inside a nanopore, hydrodynamic and confinement-induced effects as well as ions moving along the DNA strand might also have to be considered for comprehensive modeling. Figure 26 shows that the presence of DNA in the nanopore leads to an increase in the current of about $40 \mathrm{pA}$ rather than a decrease, and we explain this phenomenon subsequently.

\section{Current decrease and increase}

The principle of particle counting is based on the Coulter counter type device (DeBlois et al., 1976), which consists of a small opening separating two electrolyte solutions and makes use of an increase in the aperture resistance when a particle passes. Particle size detection is related to the size of the opening. Micromachined Coulter counters have been fabricated (Koch et al., 1999; Saleh and Sohn, 2001, 2002) and molecular Coulter counters are under investigation (Kobayashi and Martin, 1997; Sun and Crooks, 2000; Ito et al., 2003). The binding of antibodies to the surface of latex colloids has been reported to be detectable upon translocation through a nanopore because of the increased particle size (Saleh and Sohn, 2003b; Carbonaro and Sohn, 2005). To do this, these authors used the highly sensitive resistive pulse method with the four-point technique (Saleh and Sohn, 2003a), where one set of the electrodes applies the voltage and the other measures the current.

Similarly to the Coulter counter, blockade of the current has been identified as the detection mechanism when DNA molecules pass through a nanopore at high ionic strength, confirmed by an increase in the blocked current amplitude when portions of the DNA molecule are folded on themselves (Chen, Gu, Brandin, et al., 2004). The observed current decrease cannot solely be explained by a blockage, and an effect that electrostatically amplifies channel narrowing has to be considered (Bonthuis et al., 2006). Because ions in the channel have a higher self-energy compared to ions in the bulk, a nanopore represents an energy barrier that increases the blockade current.

Interestingly, current decreases and increases result when DNA molecules pass through a nanopore, as demonstrated by Chang et al. (2004). In a solid-state 4-nmdiam pore at an ionic strength of $0.1 \mathrm{M} \mathrm{KCl}$, a current increase has been measured upon DNA translocation, which has been explained by the surface current in the pore. Fan et al. (2005) demonstrated a transition from current decrease to current increase during DNA passage by changing the buffer ionic strength, and an interplay between electrostatic charge and geometric blocking effects has been suggested. The current enhancement has been attributed to the introduction of counterions in the nanotube due to the charge on the DNA molecules. This observation was confirmed by Smeets, Keyser, Krapf, et al. (2006), who described two competing effects to take into consideration in constructing a model. On one hand, conductance is decreased because of the volume occupied by the DNA molecule, lowering the number of available charge carriers for ionic transport. On the other hand, counterions shielding the charge of the DNA backbone add a positive contribution to the ionic current. Taking both effects into account, the change in the conductance $\Delta G$ 

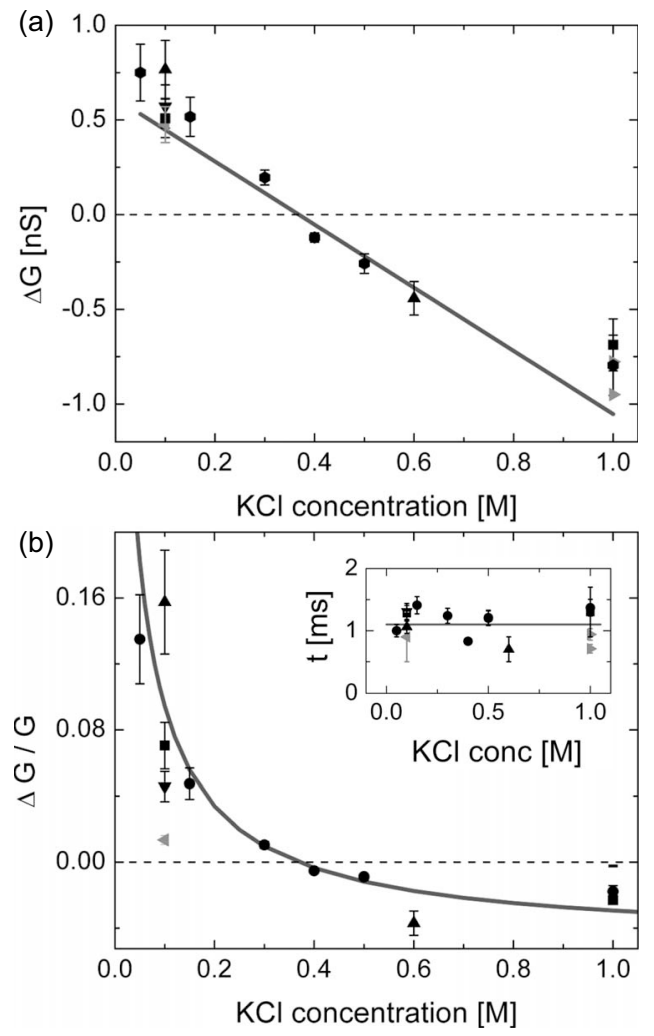

FIG. 27. Transition from current increase to current decrease for DNA translocations through a nanopore. (a) Conductance changes $\Delta G$ and (b) relative conductance changes $\Delta G / G$ due to DNA molecule translocation for $\mathrm{KCl}$ concentrations between $50 \mathrm{~m} M$ and $1 M$. Each symbol represents an individual pore, and the line is a linear fit to the data. The line in (b) has been calculated for a change in surface charge as a function of the salt concentration. The inset shows the DNA molecule translocation time as a function of $\mathrm{KCl}$ concentration. From Smeets, Keyser, Krapf, et al., 2006.

due to DNA molecule translocation in a $\mathrm{KCl}$ solution is (Schoch, 2006; Smeets, Keyser, Krapf, et al., 2006)

$$
\begin{aligned}
\Delta G= & \frac{n_{\mathrm{DNA}}}{d}\left(-\frac{\pi}{4} d_{\mathrm{DNA}}^{2}\left(\mu_{\mathrm{K}^{+}}+\mu_{\mathrm{Cl}^{-}}\right) n_{\mathrm{KCl}} e\right. \\
& \left.+\mu_{\mathrm{K}^{+}}^{*} q_{\mathrm{DNA}_{l}}^{*}\right),
\end{aligned}
$$

where $n_{\mathrm{DNA}}$ is the number of DNA molecules in the nanopore, $d_{\mathrm{DNA}}$ is the diameter of the DNA strand, $\mu_{\mathrm{K}^{+}}^{*}$ is the effective electrophoretic mobility of potassium ions moving along the DNA strand, and $q_{\mathrm{DNA}_{l}}^{*}$ is the effective charge on the DNA molecule per unit length, which is assumed to be constant. Smeets, Keyser, Krapf, et al. (2006) calculated that $\mu_{\mathrm{K}^{+}}^{*} q_{\mathrm{DNA}_{l}}^{*}=2.09$ $\times 10^{-17} \mathrm{~m} \Omega^{-1}$, which implies a reduced number of electrons per base pair (Rabin and Tanaka, 2005). The crossover concentration (Fig. 27) is obtained when $\Delta G=0$ in Eq. (62), and this parameter is independent of the nanopore length $d$.

Current increase during DNA translocation through a nanopore at low ionic strength has also been computed using a multi-ion model that accounts for the polarization of the EDL (Liu et al., 2007), and these theoretical predictions are in qualitative agreement with experimental observations.

\section{B. Nanowire sensors}

Similar to nanopores, nanowires are one-dimensional nanostructures that show detectable electrical changes upon interactions with molecules. Such unique properties are possible because the interactions have an effect on a significant part of the nanopore or nanowire, which is related to their large surface-to-volume ratio. The reader may perceive that nanowires have electrical effects similar to those of previously described nanochannels. Such nanometer-scale phenomena have been exploited to demonstrate sensitive, label-free, and realtime electrical detection with the integration of nanowires in $\mu \mathrm{TAS}$, which has high potential for use in health care, medicine, and life sciences, and has been described in recent reviews (Lieber, 2003; Ramachandran et al., 2005; Gooding, 2006; Patolsky et al., 2006a, 2006b; Carlen and Van den Berg, 2007). However, the reasons for fast biomolecule detection at low analyte concentrations are not yet understood, as described in Sec. V.B.4, and further research in nanofluidics is necessary to explain such observations.

Carbon nanotubes have remarkable electrical properties (Tans et al., 1998; Dekker, 1999) and can be used as electrochemical sensors in liquids (Wang, 2005; Vamvakaki et al., 2006; Zhou et al., 2007). It has naturally been assumed that the observed conductance change is due to the doping of the nanotube by adsorption of molecules on its surface (Collins et al., 2000; Chen et al., 2003; Star et al., 2006). However, Heinze et al. (2002) demonstrated that carbon nanotubes operate as unconventional Schottky barrier transistors, in which switching occurs primarily by modulation of the contact resistance rather than channel conductance; this has been confirmed by biomolecule detection studies (Chen, Choi, Bangsaruntip, et al., 2004; Tang et al., 2006). Thus, the contact resistance of the nanotube-electrode interface mainly (unintentionally) determines the device operation. In contrast, semiconductor nanowires interfaced with low-bias ballistic contacts behave as preferential field-effect transistors. Additionally, applications of high-performance nanotubes have been hindered by difficulties in producing uniform semiconducting nanotubes, a consideration that is not limited to nanowires.

\section{Fabrication techniques}

Nanowires can be fabricated using both "top-down" and "bottom-up" methods, but the latter techniques are less well suited for bulk production, which would hinder widespread application of the technology. In order to realize fully integrated detection systems, the fabrication of nanowires with top-down methods is preferential and has been considered to build complementary metaloxide-semiconductor compatible nanowires with high 


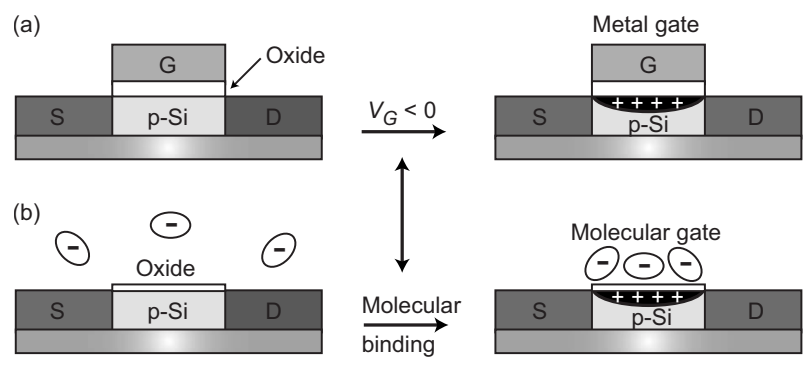

FIG. 28. Operation mechanism of nanowire sensors. (a) Schematic of a metal-oxide-semiconductor field-effect transistor, where S, D, and G are the source, drain, and gate metal electrodes, respectively. If a negative gate potential $V_{G}$ is applied, holes are accumulated in $p$-type silicon ( $p$-Si), leading to a conductance increase. (b) Schematic of an ion-sensitive field-effect transistor, in which a conductance increase is measurable upon binding of negative molecules to the surface of $p$-Si. Adapted from Patolsky et al., 2006b.

performance (Stern, Klemic, Routenberg, et al., 2007), as well as two- and three-dimensional assemblies of nanowires (Jin et al., 2004; Ahn et al., 2006; Javey et al., 2007; Yu et al., 2007).

Bottom-up fabrication methods (Morales and Lieber, 1998) are favored for electric transport studies in semiconductor nanowires, and these structures have been grown with diameters down to a few nanometers (Ma et al., 2003; Xia et al., 2003; Fan et al., 2006). Silicon is the most commonly used semiconductor nanowire material, but other materials also show field-effect transistor characteristics (Wu et al., 2004; Wang and Dai, 2006). Crystallization is essential to create a nanowire, and is composed of droplet formation, nucleation, and growth, for which the vapor-liquid-solid growth method seems to be the most versatile. Fundamental electron transport investigations of grown nanowires with diameters in the range of $40-200 \mathrm{~nm}$ have been performed, showing Coulomb blockade resulting from single-charge tunneling through a single quantum structure with discrete energy levels (De Franceschi et al., 2003; Thelander et al., 2003; Bjork et al., 2004; Zhong et al., 2005). For future nanoelectronic devices, hole and electron doping of silicon nanowires have been examined to increase their performance (Cui et al., 2003; Zheng et al., 2004), and germanium/silicon core/shell heterostructures have demonstrated enhanced gate coupling (Xiang et al., 2006; Liang et al., 2007).

\section{Operation mechanism}

The operation mechanism of nanowire sensors is based on the principle of a field-effect transistor, relying on a controllable conductivity between the source and the drain upon change in the gate potential. The most commonly used field-effect transistor is the metal-oxidesemiconductor field-effect transistor as shown in Fig. 28 (a), with the semiconducting element of a $p$-type silicon $(p-\mathrm{Si})$ connected to a current injecting source and collecting drain metal electrodes. The charge concentration of the $p$-Si can be controlled by the gate electrode, which is capacitively coupled via an oxide layer. Thus, if a negative gate voltage is applied, the accumulation of holes is obtained in $p$-type silicon at the opposite side of the capacitor, leading to an increased conductance for a fixed source-drain voltage. A positive gate voltage results in an increased number of electrons in $p$-Si, which depletes holes and thereby decreases the conductance.

Conductivity changes between source and drain can be induced by a gate electrode, as well as by binding molecules to the surface of a semiconductor, and these changes lead to charge concentration modulations of $p$-Si. Such devices are denoted ion-sensitive field-effect transistors and respond to the binding of, for example, negatively charged molecules to the surface of $p$-type silicon [Fig. 28(b)], leading to an accumulation of holes between the source and the drain, and consequently an increase in device conductance. This concept was described years ago (Bergveld, 1972), but high detection sensitivities were achieved only when the cross-sectional area of the $p$-Si was reduced to tens of nanometers (Cui et al., 2001). By reducing the size of $p$-Si, the charge accumulation or depletion in the nanowire occurs within a significant portion of the one-dimensional structure, leading to increased detection sensitivities. Sizedependent properties of nanowires have been reported by Giovine et al. (2001) and Li et al. (2005).

For the selective detection of macromolecules, it is essential to control the ionic strength of the buffer. Stern, Wagner, Sigworth, et al. (2007) demonstrated that if the net charge of biomolecules is screened in a high ionic strength solution, the measured signal was negligible, while in experiments at low ionic strength, the majority of the macromolecule's net charge was unscreened.

\section{Single-molecule sensitivity}

Nanowires are coated with a recognition group at their surface to achieve specific binding with biomolecules. The working principle has been demonstrated using nanowires as $p \mathrm{H}$ sensors, showing a linear conductance over the $p \mathrm{H}$ range of 2 to 9 , which is understood in terms of the change in surface charge during protonation and deprotonation (Cui et al., 2001). In addition to chemical species, various biomolecules have been sensed down to femtomolar concentrations, such as DNA and DNA enzymatic processes (Hahm and Lieber, 2004; Li, Chen, Li, et al., 2004), proteins (Zheng et al., 2005; Gamby et al., 2006), drugs (Wang, Chen, Lin, et al., 2005), and single viruses (Patolsky et al., 2004). Nanowire transistor arrays (Huang et al., 2001) allow the detection and stimulation of neuronal signals, showing their applicability for real-time cellular assays (Patolsky, Timko, Yu, et al., 2006). Identifying a number of molecular markers is required for disease diagnosis, obtained with multiplexed detection (Koehne et al., 2004; Lin et al., 2007). Zheng et al. (2005) showed that false positive signals can be discriminated by correlating the response over time from two types of device elements, which are either nanowires modified with different functionaliza- 

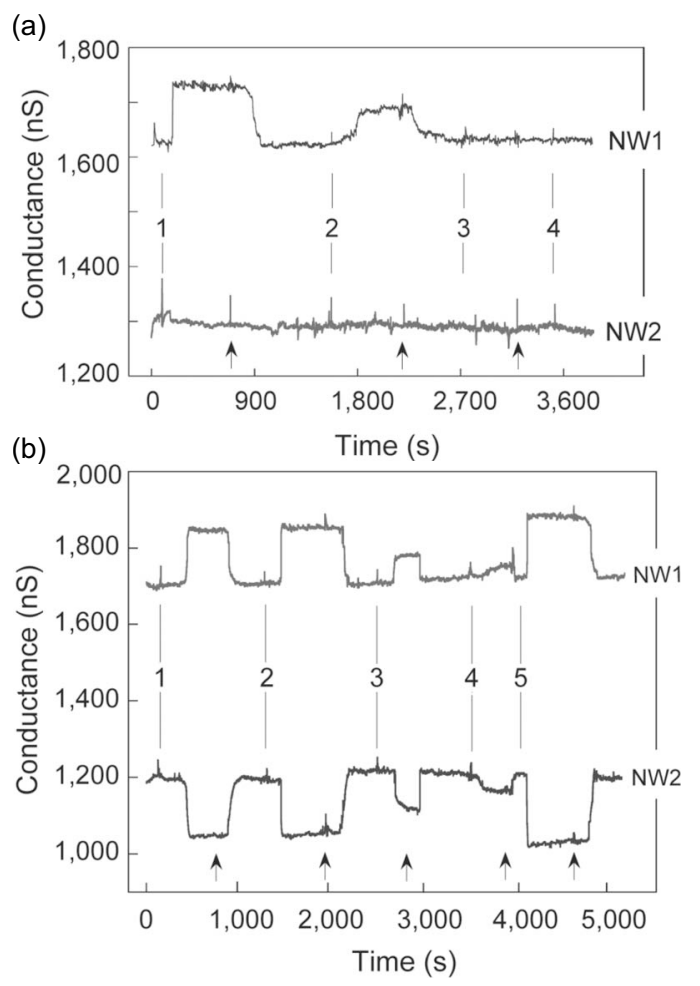

FIG. 29. Multiplexed detection with nanowire arrays. (a) Conductance vs time data were recorded simultaneously for two $p$-type silicon nanowires (NW). NW1 was functionalized with prostate specific antigen (PSA) antibody 1, and NW2 was modified with ethanolamine. Vertical lines correspond to the addition of (1) $9 \mathrm{pg} \mathrm{ml}^{-1}$ PSA, (2) $1 \mathrm{pg} \mathrm{ml}^{-1}$ PSA, (3) $10 \mu \mathrm{g} \mathrm{ml}^{-1}$ bovine serum albumin, and (4) a mixture of $1 \mathrm{ng} \mathrm{ml}^{-1}$ PSA and $10 \mu \mathrm{g} \mathrm{ml}^{-1}$ PSA antibody 1. (b) Complementary sensing of PSA using the $p$ - and $n$-type nanowires NW1 and NW2, respectively. Vertical lines correspond to times when PSA solutions with the following concentrations were added: (1) $0.9 \mathrm{ng} \mathrm{ml}^{-1}$; (2) $0.9 \mathrm{ng} \mathrm{ml}^{-1}$; (3) $9 \mathrm{pg} \mathrm{ml}^{-1}$, (4) $0.9 \mathrm{pg} \mathrm{ml}^{-1}$, and (5) $5 \mathrm{ng} \mathrm{ml}^{-1}$. Black arrows correspond to points where the solution was switched from protein to pure buffer solutions. From Zheng et al., 2005.

tions [Fig. 29(a)] or the incorporation of $p$ - and $n$-type nanowires in a single chip [Fig. 29(b)]. Thus, multiplexing is robust against false positive signals arising from nonspecific binding or electronic noise. Demultiplexers have also been realized (Beckman et al., 2005), which are electronic circuits designed to separate combined signals.

\section{Detection efficiency}

The surface area of a nanowire in contact with a liquid is small, which could lead to a limited overall sensitivity for the detection of analytes at low concentrations. Sheehan and Whitman (2005) calculated that a $10-\mu \mathrm{m}$-long hemicylindrical sensor with a diameter of a few tens of nanometers needs an accumulation time under static diffusion in a $1 \mathrm{f} M$ analyte solution of $\sim 1 \mathrm{~h}$ for the first molecule, $\sim 1$ day for the tenth molecule, and $\sim 1$ week for the 100th molecule. The improvement when the sample flows past the sensor is pronounced for micrometer-sized detectors, but only marginal for nanoscale sensors. Thus, the detection efficiency at low analyte concentrations is likely limited by species transport to the sensor (Krapf, Quinn, Wu, et al., 2006) rather than by its signal transduction or noise (Reza et al., 2006).

However, Fig. 29 demonstrates that the detection of biomolecules is much faster than calculated by Sheehan and Whitman (2005). Zheng et al. (2005) reported that electrokinetic effects lead to an enhancement in the local concentration of species, such as dielectrophoretic trapping of biomolecules (Hughes, 2000; Morgan and Green, 2003; Wong et al., 2004; Li et al., 2006). Even faster detection response times have been reported by Stern, Wagner, Sigworth, et al. (2007); they attributed this to their fluid injection system, which is not laminar and does not rely on diffusion to transport analyte molecules to the nanowires. Further investigation is necessary to predict the detection times and limits of nanowire sensors and deduce the binding kinetics.

\section{FABRICATION OF NANOCHANNELS}

Transport through nanoporous systems has been investigated in membrane science for decades (Helfferich, 1962), and puzzling processes still exist. This might be due to the geometry and surface condition of nanopores in membranes, characterized by parameters such as pore length, deviation of pore shape from perfect cylinders, and pore size distribution, which are difficult to deduce and control.

In order to overcome the limitations of nanoporous membranes and accurately describe transport phenomena in nanofluidics, single solid-state nanometer-sized openings are required for experimental investigations. Their geometry and surface properties have to be well defined in order to model transport through nanochannels or study molecular dynamics in confined spaces. Such requirements can be satisfied with microfabrication techniques (Madou, 1997) used for the realization of nanochannels on a chip. Various methods of producing nanochannels exist and are divided into the categories of top-down and bottom-up fabrication methods (Mijatovic et al., 2005). We focus here on the well-established topdown processes for the fabrication of channels with the smallest dimension below $100 \mathrm{~nm}$. Additionally, promising fabrication techniques to produce single nanopores in membranes are described.

Fabrication technologies do not limit the realization of nanostructures, but they have to be connected to the macroscopic world. Nanochannels on a chip can be accessed by a consecutive increase in the size of channels from nanometer to micrometer and millimeter dimensions. It has been recommended to design the system in such a way that the nanochannel can be operated with the feed flow perpendicular to the flow direction in the nanochannel, reducing the risk of fouling or filter cakes. We particularly emphasize nanochannels fabricated with Pyrex because this material is hydrophilic, which allows for easy filling of the channels by capillary forces; nonconductive, which is ideal for high-resistance measure- 
ments; rigid with minimal surface deflections and nondeformable even at high pressures within nanochannels (Tas et al., 2003); and transparent, which facilitates fluorescence experiments and allows for further biochemical surface modifications.

Despite these advantages, it has to be considered that, although glass is one of the most chemically inert materials, the dissolution rate of Pyrex in water is $10^{-11} \mathrm{~cm} / \mathrm{s}$ at room temperature (Doremus, 1994; Devreux et al., 2001). This could be a problem for nanometer-sized apertures, since $10 \mathrm{~nm}$ of glass are dissolved in $28 \mathrm{~h}$. In fact, the interaction between water and the silica network is not limited to the surface, but can also occur behind the glass-water interface (Scholze, 1988), resulting in a loose, swelled, and unstructured layer at the surface of glass that is denoted as a silica gel. Compared to bulk glass, this gel layer has an enhanced ion mobility, lower electric resistance, different $\mathrm{Si}-\mathrm{O}$ bond lengths, and faster dehydration (Wikby, 1974; Yaroshchuk, 2001a). The long-term stability and controllability of glass is therefore limited, but can be improved with surface pretreatment (Hench and Clark, 1978; Dilmore et al., 1979) or layer deposition. Atomic layer depositions allow fine-tuning surface properties (Jones and Chalker, 2003; Farmer and Gordon, 2006; Wanunu and Meller, 2007) or the size of nanopores (Elam et al., 2003; Chen, Mitsui, Farmer, et al., 2004). Layer-by-layer depositions are used to deposit polyelectrolytes with alternating charges on top of one another (Decher and Schlenoff, 2002), and the layer properties can be precisely controlled (Krasemann and Tieke, 2000; Schwarz and Schonhoff, 2002; Huebsch et al., 2004; Lulevich and Vinogradova, 2004; Poptoshev et al., 2004; Sui and Schlenoff, 2004; Lee et al., 2006). Such polyelectrolytes have been applied to microchannels (Dutta and Ramsey, 2004), capillaries (Graul and Schlenoff, 1999), and nanopores (Vorrey and Teeters, 2003; Alem et al., 2007).

Different nanochannel fabrication methods allow for the realization of one-dimensional (1D) and twodimensional (2D) nanochannels. If the characteristic nanometer-scale dimension in a slitlike 2D nanochannel is defined with etching or a sacrificial layer, conventional optical lithography, for example, can be used for patterning. This technique establishes features with a minimal size that is almost the same as the exposure wavelength (Okazaki, 1991), typically in the micron range. The growing market in integrated circuits has led to resolution enhancement technologies, which allow the manufacturing of feature sizes down to several tens of nanometers (Schellenberg, 2005). Conventional photolithography has remained the standard at most research institutes, so nanochannels defined in two dimensions on the nanometer scale are fabricated with high-energy beams for research purposes. In comparison to optical lithography where whole wafers can be exposed at once for bulk production, high-energy beams can only be used locally.

2D nanochannels are usually used for research purposes because nanofluidic transport phenomena already occur if one dimension of the nanochannel's cross sec-

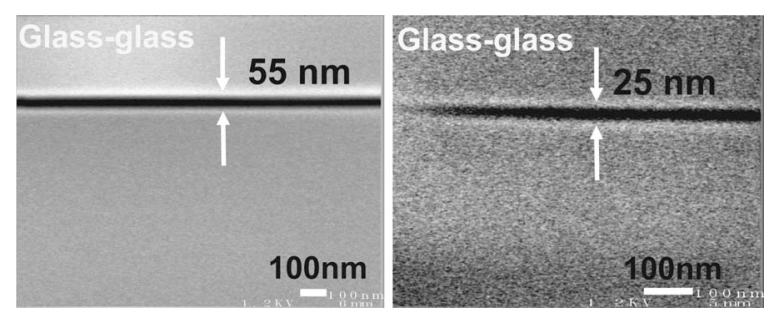

FIG. 30. Cross-sectional scanning electron microscope images of nanochannels with heights of 55 and $25 \mathrm{~nm}$ fabricated in a glass substrate and bonded with another glass cover. From Mao and Han, 2005.

tion is on the nanometer scale, they can be produced with standard microfabrication technologies, and the throughput of liquids is higher than if both dimensions are nanometer sized.

\section{A. Wet and dry etching}

2D nanofluidic channels with the smallest dimension of about 20-25 $\mathrm{nm}$ have been developed (Mao and Han, 2005). The nanometer-sized dimension of the channel is obtained by etching a nanometer-high recess in glass or silicon, followed by a bonding process to a glass substrate to form the nanofluidic channel (see Fig. 30). Buffered oxide etch has a stable etch rate and has been used for precise bulk micromachining in glass, whereas reactive ion etching has been applied on silicon substrates. For the formation of 2D nanochannels, glass-glass fusion bonding (at $550{ }^{\circ} \mathrm{C}$ ) or glass-silicon anodic bonding have been used. Haneveld et al. (2003) used anisotropic etching of $\langle 110\rangle$ silicon resulting in channel sidewalls that are precisely perpendicular to the wafer surface. It has to be considered that glass etching processes lead to an unintentional increase in surface roughness (Zeze et al., 2002).

\section{B. Sacrificial layer methods}

Nanochannels can be made with a nanometer-thick sacrificial layer that is first used to define the male form of the nanochannel and is then removed in order to open the aperture (Kittilsland et al., 1990). This method was used to construct nanofilters in silicon membranes with silicon dioxide as a sacrificial layer, demonstrated by Chu et al. (1999) and used for biomedical applications (Desai et al., 1999; Tu et al., 1999; Martin and Grove, 2001). Long nanochannels on a silicon substrate have been realized by the fabrication of metal nanowires covered with a silicon nitride capping layer and subsequent metal line etching. Tas et al. (2002) used the fabrication of nanowires on the sidewall of a submicrometer step (Fig. 31), whereas Peng et al. (2003) applied electronbeam lithography to control the nanoscale dimension of the wires. Nanochannels have also been produced by the adherence of a capping polysilicon layer to the bottom layer after removal of a sacrificial strip (Tas et al., 2002). 

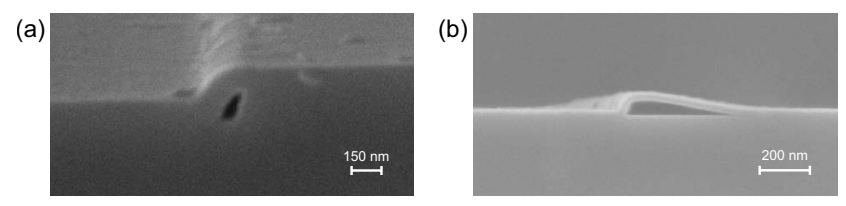

FIG. 31. Scanning electron microscope images of 2D defined nanochannels fabricated by two different sacrificial layer methods. (a) Formation of a nanowire on the sidewall of a step and (b) etching of a sacrificial strip separating the substrate and the capping layer. From Tas et al., 2002.

Centimeter-long nanochannels can be fabricated with a sacrificial $\mathrm{SiO}_{2}$ layer (Lee et al., 2003; Han, de Rooij, and Staufer, 2006).

Sacrificial layers can be used as nanometer-sized spacers between two substrates to define their separation distance. For example, by patterning a layer of amorphous silicon with photolithography, 2D nanochannels with heights of $50 \mathrm{~nm}$ have been realized in Pyrex (Schoch et al., 2005). This method allows convenient contact of the nanochannel with two microchannels, which are fabricated by wet etching (Fig. 32).

\section{High-energy beam techniques}

The focused ion beam is a powerful tool used to directly fabricate structures on the substrate down to some tens of nanometers. This instrument has been used to mill or etch 1D nanochannels in different types of materials such as glass (Wang, Tegenfeldt, Reisner, et al., 2005; Schoch, 2006), silicon (Campbell et al., 2004), and poly(methyl methacrylate) (Cannon et al., 2004).

In electron-beam lithography, the pattern is directly exposed to the resist [or ice layer (King et al., 2005)] on the substrate, and structures are then obtained with bulk micromachining. For example, this technique has been used to produce monolithic nanofluidic sieving structures that are similar to two-dimensional artificial gels used for DNA molecule manipulations (Volkmuth and Austin, 1992; Turner et al., 1998). These structures are also useful for interfacing microfluidic and nanofluidic channels (Cao et al., 2002). An asymmetric lattice of orthogonally interconnected nanochannels with equal pitch and dissimilar channel cross section has been fabricated using electron-beam lithography by Riehn et al. (2006), which could be used for the separation of DNA molecules (Sec. IV.D).

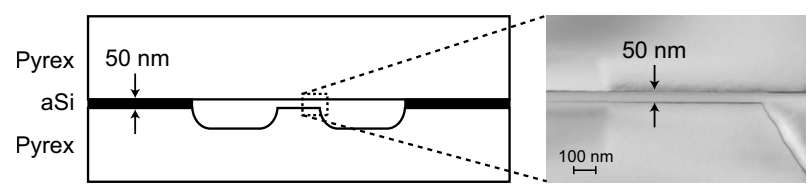

FIG. 32. Cross section of a chip illustrating two microchannels linked by a nanochannel, whose height is defined by the thickness of the amorphous silicon $(a-\mathrm{Si})$ layer. The inset shows a scanning electron microscope image of the 50-nm-high nanochannel. Adapted from Schoch, 2006.

\section{Nanoimprint lithography}

A low-cost and high-throughput method for the fabrication of 2D nanofluidic channels is nanoimprint lithography, which was first reported by Chou and Krauss (1997). In a single step with elevated temperature and pressure, a channel template can be imprinted onto a thin polymer film cast on a glass cover slip (Quake and Scherer, 2000; Guo et al., 2003; Cheng and Guo, 2004) or onto a poly(methyl methacrylate) wafer (Abgrall et al., 2007). The nanochannel dimensions are controlled by a simple relationship involving the initial polymer layer thickness and the mold pattern configuration, and the grating period can be reduced by frequency doubling (Cui et al., 2007). Nanoimprint lithography has also been demonstrated on free-standing membranes (Heyderman et al., 2003).

\section{E. Bonding and sealing methods}

To achieve encapsulation of bulk-machined nanochannels, bonding and sealing has to be performed. Depending on the bulk material in which the nanochannels have been produced (Madou, 1997), different methods can be used. Two silicon wafers can be joined by silicon fusion bonding at temperatures above $1000{ }^{\circ} \mathrm{C}$. For the fabrication of silicon-on-glass wafers, anodic bonding is known to result in high bonding strength when simultaneously exposing the wafers to an elevatedtemperature treatment of about $500{ }^{\circ} \mathrm{C}$ and a dc voltage of approximately $750 \mathrm{~V}$ (Knowles and van Helvoort, 2006). Methods for bonding between two glass wafers can be divided into methods using either high temperature (glass fusion bonding), extremely high pressures (Sayah et al., 2000), or chemicals such as hydrofluoric acid (Nakanishi et al., 2000; Chen et al., 2006). Fabrication processes at lower temperatures have been developed because high temperatures are not suitable for heat-sensitive materials such as electrodes.

Glass wafers with an intermediate layer of amorphous silicon can be bonded at temperatures of about $250{ }^{\circ} \mathrm{C}$ and a dc voltage of approximately $500 \mathrm{~V}$ (Berthold et al., 2000; Lee et al., 2001). This field-assisted bonding technique is interesting for microelectronic devices, since encapsulation is achieved at reasonable temperatures. Other intermediate or adhesive layers (Niklaus et al., 2006) have been used for wafer bonding, such as water glass (Satoh, 1999; Ito et al., 2002), spin-on glass (Wang et al., 1997), benzocyclobutene (Niklaus et al., 2001), or glue (Sayah et al., 2000). However, using such adhesive layers for the fabrication of nanochannels bears the risk of nanochannel clogging.

\section{F. Realization of single nanopores in membranes}

The realization of single nanopores fabricated in a membrane are not the principal focus of this review, but we present selected techniques in this area. The integration capabilities of membranes in $\mu \mathrm{TAS}$ are growing 

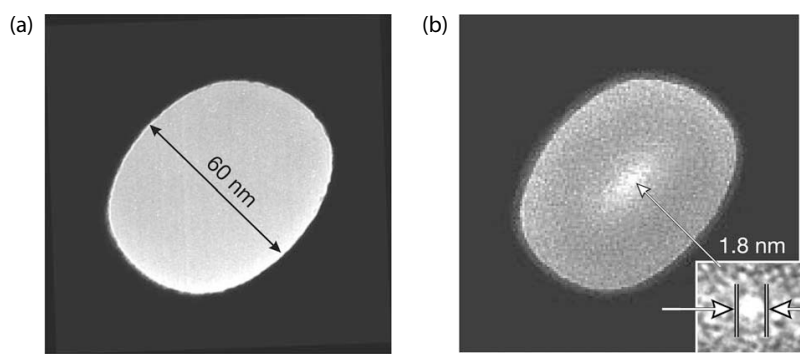

FIG. 33. Transmission electron microscope image of (a) initial 60 -nm-diam pore and (b) the same sample after ion beam exposure yielding a shrunken 1.8-nm-diam pore. From Li et al., 2001.

(Kuo et al., 2003; de Jong et al., 2006; Flachsbart et al., 2006), and solid membranes produced in a wafer seem to be the most suitable for this.

The smallest single nanopore that can be achieved in a solid is fabricated with high-energy beams, and is promising for DNA translocation measurements as presented in Sec. IV. For its fabrication, a $60-\mathrm{nm}$-diam pore was made with a focused ion beam on a 500-nm-thick silicon nitride membrane. Li et al. (2001) exposed this pore to a $3 \mathrm{keV} \mathrm{Ar}^{+}$ion beam, which decreased the initial pore size of $60 \mathrm{~nm}$ down to $1.8 \mathrm{~nm}$ (Fig. 33). In this method, called "ion beam sculpting," the atomic flow of matter into the pore occurs upon stimulation by the ion beam. Storm et al. (2003) used a transmission electron microscope to shrink a pore. First, a $20 \mathrm{~nm}$ pore was fabricated in a 350-nm-thick silicon membrane using electron-beam lithography and anisotropic etching. After thermal oxidation, the pore was reduced to $2 \mathrm{~nm}$, since the silicon oxide can be fluidized upon exposure to a high-energy electron-beam. It is proposed that Jouleheating-assisted irradiation-induced diffusion is the reason for such shrinkage (Zhang et al., 2007).

A different technique can be used to produce synthetic nanopores with diameters of $1.8 \mathrm{~nm}$ in $10-\mathrm{nm}$ thick membranes, and is based on drilling (Storm et al., 2003; Ho et al., 2005). Single nanopores in a variety of membrane materials have been achieved with electronbeam-stimulated decomposition and sputtering using a transmission electron microscope operated at 200-300 keV, which generates a tightly focused beam as small as $0.5 \mathrm{~nm}$ in diameter. Such pores in silicon nitride have been reduced in size by Krapf, Wu, Smeets, et al. (2006) upon exposure to a low-intensity electron beam, allowing shrinkage down to a 0.8 -nm-diam opening, representing the smallest reported nanopore. Monolayer deposition of silicon oxide to shrink an initial 50-nmdiam pore down to $4 \mathrm{~nm}$ in a silicon nitride membrane was demonstrated by Danelon et al. (2006). These pores are suitable for further biochemical surface modifications.

Single and asymmetric nanopores with diameters down to $2 \mathrm{~nm}$ can be fabricated in a polymer foil using the track etching technique, based on the irradiation of a polymer foil with energetic heavy ions and subsequent chemical etching (Siwy and Fulinski, 2002a; Harrell et al., 2003; Siwy, Apel, Dobrev, et al., 2003; Li, Harrell, and Martin, 2004). Fast heavy ions can lead to permanent material changes along the nearly straight ion path, referred to as ion tracks, which can be selectively opened with appropriate etchants. Asymmetric pores have been shown to exhibit unique properties, specifically asymmetric diffusion (Siwy, Kosinska, Fulinski, et al., 2005), ionic current rectification (see Sec. III.A.3) (Wei et al., 1997; Siwy et al., 2004; Cervera et al., 2006; Vlassiouk and Siwy, 2007) by using calcium (Siwy et al., 2006) or DNA (Harrell et al., 2004) to change the surface properties, voltage gating similar to biological channels (Siwy, Apel, Baur, et al., 2002, 2003), and sensing of proteins (Siwy, Trofin, Kohli, et al., 2005).

More recently, multiple nanopores with controlled sizes between 5 and $25 \mathrm{~nm}$ were fabricated in a 15-nmthick nanocrystalline silicon membrane, which has remarkable mechanical properties (Striemer et al., 2007). Nanopores are obtained from voids formed spontaneously as nanocrystals of the membrane nucleate and grow during rapid thermal annealing. Currently, this process cannot be predicted with existing models.

\section{CONCLUSIONS AND PERSPECTIVES}

This review shows that nanofluidics offers a variety of unique properties in nanochannels, nanofilters, nanopores, nanowires, and at the microchannel-nanochannel interface. In such nanostructures, transport phenomena that are accessible only at these length scales can be achieved, and this opens new opportunities and research directions. Thus, nanofluidics is significantly relevant to nanoscience and nanotechnology, and may lead to new advances.

The nanostructures presented have been kept relatively simple in order to elucidate their physical principles, establishing a basis for massive parallelism with integrated electrical characterizations for potential nanoelectrofluidic systems. Such devices have a high potential for impact in biological and medical applications for separation, detection, and analytic purposes. Nanofluidic systems have the capability of dealing with a limited amount of sample, which is particularly important in biomedicine, for example, to analyze species from a single cell. Important practical significance is attributed to the substantial decrease in the immunoassay response time, demonstrated in nanochannels with an induced flow (Schoch et al., 2007).

In addition to the elucidated properties of nanofluidic systems, chemical and physical properties of single molecules can be investigated in nanochannels (Craighead, 2006). Such single-molecule interrogations have demonstrated restriction mapping of DNA molecules (Riehn et al., 2005) and repressor-DNA interactions (Wang, Tegenfeldt, Reisner, et al., 2005). Dependent on the confinement degree of DNA in nanochannels, diffusivity (Balducci et al., 2006) and stretching (Tegenfeldt, Prinz, Cao, Chou, et al., 2004; Reisner et al., 2005; Jo et al., 2007; Krishnan et al., 2007) have been studied at different conditions to describe the fundamental properties of 
these polymer molecules. For single-molecule analysis, optical techniques are the most straightforward, standard fluorescence microscopy is typically used, and fluorescence correlation spectroscopy (Magde et al., 1972; Hess et al., 2002) in submicrometer-sized channels (Foquet et al., 2002, 2004) and near-field scanners with high spatial resolution (Tegenfeldt et al., 2001; Tegenfeldt, Prinz, Cao, Huang, et al., 2004) are also considered.

\section{ACKNOWLEDGMENTS}

This review originates from the Ph.D. thesis of Schoch (2006) and his work as a Postdoctoral Associate, and we therefore gratefully acknowledge support from the program "Doktorieren an der anderen ETH" and the DuPont-MIT Alliance.

\begin{tabular}{|c|c|c|c|}
\hline LISTS & REVIATIO & & \\
\hline $\mathrm{CPE}$ & constant phase element & & $d_{\text {DNa }}$ \\
\hline DBL & diffusion boundary layer & & $d_{\mathrm{OHP}}$ \\
\hline EDL & electrical double layer & & $f$ \\
\hline EEE & exclusion-enrichment effect & & $f$ \\
\hline$\mu \mathrm{TAS}$ & micro total analysis systems & & $J_{s}$ \\
\hline$A$ & surface area & $\mathrm{m}^{2}$ & 要 \\
\hline$C_{\mathrm{CPE}}$ & CPE capacitance & $\mathrm{F}$ & \\
\hline$C_{\mathrm{GC}}$ & Gouy-Chapman layer capacitance & $\mathrm{F}$ & $n_{\mathrm{CPE}}$ \\
\hline$C_{H}$ & Helmholtz layer capacitance & $\mathrm{F}$ & $n_{\text {DNA }}$ \\
\hline$C_{\text {stray }}$ & stray capacitance & $\mathrm{F}$ & $\begin{array}{l}n_{i} \\
\infty\end{array}$ \\
\hline$D$ & diffusion coefficient & $\mathrm{m}^{2} \mathrm{~s}^{-1}$ & $n_{i}$ \\
\hline E & electric field & $\mathrm{V} \mathrm{m}^{-1}$ & $\mathbf{p}$ \\
\hline$E_{s}$ & electric field in nanochannel & $\mathrm{V} \mathrm{m}^{-1}$ & $q_{*}$ \\
\hline$E_{t}$ & tangential electric field & $\mathrm{V} \mathrm{m}^{-1}$ & $q_{\text {DNA }}^{*}$ \\
\hline$F_{e}$ & entropic force & $\mathrm{kg} \mathrm{m} \mathrm{s}^{-2}$ & $q_{\mathrm{DNA}_{l}}^{*}$ \\
\hline$F_{\mathrm{el}}$ & electrical force & $\mathrm{kg} \mathrm{m} \mathrm{s}^{-2}$ & $r$ \\
\hline$F_{\text {ot }}$ & optical force & $\mathrm{kg} \mathrm{m} \mathrm{s}^{-2}$ & $r_{0}$ \\
\hline$G$ & conductance & $\mathrm{S}$ & $r_{D}$ \\
\hline$G_{p}$ & conductance plateau & $\mathrm{S}$ & $t$ \\
\hline$I$ & current & A & $t_{\mathrm{an}}$ \\
\hline$I_{d}$ & current density & $\mathrm{A} \mathrm{m}^{-2}$ & $t_{\mathrm{ca}}$ \\
\hline$I_{d_{0}}$ & exchange current density & $\mathrm{A} \mathrm{m}^{-2}$ & $t_{d}$ \\
\hline$I_{\lim }$ & limited current & $\mathrm{A}$ & $t_{s}$ \\
\hline$I_{s}$ & ionic strength & $M$ & $\mathbf{v}_{c}$ \\
\hline $\mathbf{J}, J$ & flux per area & $1 \mathrm{~s}^{-1} \mathrm{~m}^{-2}$ & $v_{\text {eo }}$ \\
\hline$K^{L}$ & bulk conductivity & $\mathrm{S} \mathrm{m}^{-1}$ & $v_{\mathrm{eo} 2}$ \\
\hline$K^{\sigma}$ & surface conductance & $\mathrm{S}$ & $v_{\text {ep }}$ \\
\hline$K^{\sigma_{d}}$ & surface conductance of diffuse & & $w$ \\
\hline & layer & $\mathrm{S}$ & $x$ \\
\hline$K^{\sigma_{i}}$ & surface conductance of Stern layer & S & $x_{f}$ \\
\hline$L$ & mean drift distance & $\mathrm{m}$ & $x_{p}$ \\
\hline$N$ & DNA base-pair number & & $y$ \\
\hline$P^{*}$ & geometrical permeability & $\mathrm{m}^{3} \mathrm{~s}^{-1}$ & $z$ \\
\hline$P_{\text {eff }}$ & effective permeability & $\mathrm{m}^{3} \mathrm{~s}^{-1}$ & $z_{i}$ \\
\hline$P_{x}$ & passage rate & $\mathrm{mol} \mathrm{s}^{-1}$ & $\Phi$ \\
\hline$R_{0}$ & radius of gyration & $\mathrm{m}$ & $\Phi_{n}$ \\
\hline$R_{\mathrm{nano}}$ & resistance of nanochannel & $\Omega$ & $\Phi_{D}$ \\
\hline$R_{t}$ & charge-transfer resistance & $\Omega \mathrm{m}^{-2}$ & $\Phi_{\text {diff }}$ \\
\hline$S^{*}$ & geometrical cross section & $\mathrm{m}^{2}$ & $\Phi_{m}$ \\
\hline$S_{\text {eff }}$ & effective cross section & $\mathrm{m}^{2}$ & $\Lambda_{m}$ \\
\hline
\end{tabular}

absolute temperature $\quad \mathrm{K}$

equilibrium potential $\mathrm{V}$

applied potential $\mathrm{V}$

impedance of CPE $\quad \Omega$

Warburg impedance $\quad \Omega$

grid size $\mathrm{m}$

particle radius $\mathrm{m}$

concentration $M$

concentration at high $I_{s} \quad M$

standard molarity of $1 \quad M$

bulk concentration $\quad M$

excess mobile counterion

concentration $M$

effective concentration $\quad M$

transition concentration $\quad M$

wall charge concentration $\quad M$

length of nanochannel $\mathrm{m}$

diameter of DNA strand $\mathrm{m}$

distance to outer Helmholtz plane $\mathrm{m}$

friction coefficient $\mathrm{kg} \mathrm{s}^{-1}$

specific friction coefficient $\quad \mathrm{kg} \mathrm{m}^{-1} \mathrm{~s}^{-1}$

height of nanochannel $\mathrm{m}$

distance between base pairs $\mathrm{m}$

CPE exponent

number of DNA in nanopore

volume density $\mathrm{m}^{-3}$

bulk volume density $\mathrm{m}^{-3}$

pressure $\quad \mathrm{N} \mathrm{m}^{-2}$

net charge $e$

effective DNA $q$ per base pair $\quad e \mathrm{bp}^{-1}$

effective DNA $q$ per length $\quad \mathrm{C} \mathrm{m}^{-1}$

radial coordinate $\mathrm{m}$

radius of pore $\mathrm{m}$

Donnan ratio

time $s$

transference number of anion

transference number of cation

depth of deep region $\mathrm{m}$

depth of shallow region $\mathrm{m}$

convective velocity field $\mathrm{m} \mathrm{s}^{-1}$

electro-osmotic velocity $\mathrm{m} \mathrm{s}^{-1}$

$v_{\mathrm{eo}}$ of the second kind $\mathrm{m} \mathrm{s}^{-1}$

electrophoretic velocity $\mathrm{m} \mathrm{s}^{-1}$

width of nanochannel $\mathrm{m}$

$x$ direction $\mathrm{m}$

DNA length inside filter $\mathrm{m}$

DNA length inside pillar array $\mathrm{m}$

$y$ direction $\mathrm{m}$

$z$ direction $\mathrm{m}$

valency of ion $i$

electric potential V

applied electric potential $\mathrm{V}$

Donnan potential $\mathrm{V}$

diffusion potential $\mathrm{V}$

membrane potential $\mathrm{V}$

molar conductivity $\quad \mathrm{S} \mathrm{m}^{2} \mathrm{~mol}^{-1}$ 


\begin{tabular}{|c|c|c|}
\hline $\begin{array}{l}\Lambda_{m}^{0} \\
\alpha\end{array}$ & $\begin{array}{l}\text { limiting molar conductivity } \\
\text { constant }\end{array}$ & $\mathrm{S} \mathrm{m}^{2} \mathrm{~mol}^{-1}$ \\
\hline$\beta$ & exclusion-enrichment coefficient & \\
\hline$\beta_{\text {part }}$ & partition coefficient & \\
\hline$\delta$ & DBL thickness & $\mathrm{m}$ \\
\hline$\epsilon$ & energy & $\mathrm{J}$ \\
\hline$\epsilon_{p}$ & potential energy & $\mathrm{J}$ \\
\hline$\varepsilon_{r}$ & relative permittivity & \\
\hline$\phi$ & flux & $\mathrm{mol} \mathrm{s}^{-1}$ \\
\hline$\phi^{*}$ & geometrical flux & $\mathrm{mol} \mathrm{s}^{-1}$ \\
\hline$\phi_{\text {eff }}$ & effective flux & $\mathrm{mol} \mathrm{s}^{-1}$ \\
\hline$\gamma_{\text {bulk }}$ & bulk conductivity & $\mathrm{S} \mathrm{m}^{-1}$ \\
\hline$\gamma_{a}$ & activity coefficient & \\
\hline$\eta$ & dynamic viscosity & $\mathrm{kg} \mathrm{m}^{-1} \mathrm{~s}^{-1}$ \\
\hline$\varphi$ & streaming potential & \\
\hline$\kappa$ & Debye-Hückel parameter & $\mathrm{m}^{-1}$ \\
\hline$\lambda_{D}$ & Debye length & \\
\hline$\mu$ & mobility & $\mathrm{m}^{2} \mathrm{~V}^{-1} \mathrm{~s}^{-1}$ \\
\hline$\mu^{*}$ & effective mobility on DNA & $\mathrm{m}^{2} \mathrm{~V}^{-1} \mathrm{~s}^{-1}$ \\
\hline$\mu_{\mathrm{eo}}$ & electro-osmotic mobility & $\mathrm{m}^{2} \mathrm{~V}^{-1} \mathrm{~s}^{-1}$ \\
\hline$\mu_{\mathrm{ep}}$ & electrophoretic mobility & $\mathrm{m}^{2} \mathrm{~V}^{-1} \mathrm{~s}^{-1}$ \\
\hline$\tilde{\mu}$ & chemical potential & $\mathrm{J} \mathrm{mol}^{-1}$ \\
\hline$\tilde{\tilde{\mu}}$ & electrochemical potential & $\mathrm{J} \mathrm{mol}^{-1}$ \\
\hline$\theta$ & deflection angle deg & \\
\hline$\rho$ & charge density & $\mathrm{C} \mathrm{m}^{-3}$ \\
\hline$\sigma_{d}$ & diffuse layer charge density & $\mathrm{C} \mathrm{m}^{-2}$ \\
\hline$\sigma_{s}$ & surface charge density & $\mathrm{C} \mathrm{m}^{-2}$ \\
\hline$\tau$ & trapping lifetime & \\
\hline$\tau_{0}$ & $\tau$ at high electric field & \\
\hline$v$ & symmetry factor & \\
\hline$\omega$ & frequency & $\mathrm{Hz}$ \\
\hline$\psi$ & electric potential due to $\sigma_{s}$ & $\mathrm{~V}$ \\
\hline$\psi_{\mathrm{ct}}$ & charge-transfer overpotential & \\
\hline$\psi_{d}$ & diffuse layer potential & $\mathrm{V}$ \\
\hline$\psi_{i}$ & inner Helmholtz plane potential & V \\
\hline$\psi_{s}$ & surface potential & $\mathrm{V}$ \\
\hline$\psi_{\text {to }}$ & total overpotential & V \\
\hline$\zeta$ & zeta potential & $\mathrm{V}$ \\
\hline
\end{tabular}

Fundamental constants and units

\begin{tabular}{|c|c|c|}
\hline$F$ & Faraday constant & $96485 \mathrm{C} \mathrm{mol}^{-1}$ \\
\hline$N_{A}$ & Avogadro constant & $6.022 \times 10^{23} \mathrm{~mol}^{-1}$ \\
\hline$R$ & gas constant & $8.314 \mathrm{~J} \mathrm{~mol}^{-1} \mathrm{~K}^{-1}$ \\
\hline$e$ & charge of the electron & $1.602 \times 10^{-19} \mathrm{C}$ \\
\hline$k_{B}$ & Boltzmann constant & $1.381 \times 10^{-23} \mathrm{~J} \mathrm{~K}^{-1}$ \\
\hline$\varepsilon_{0}$ & $\begin{array}{l}\text { permittivity of free } \\
\text { space }\end{array}$ & $8.854 \times 10^{-12} \mathrm{~F} \mathrm{~m}^{-1}$ \\
\hline $\begin{array}{l}\text { Capaci- } \\
\text { tance }\end{array}$ & $\mathrm{F}=\mathrm{C}$ & $\mathrm{V}^{-1}=\mathrm{A}^{2} \mathrm{~s}^{4} \mathrm{~kg}^{-1} \mathrm{~m}^{-2}$ \\
\hline Charge & coulomb & $\mathrm{C}=\mathrm{A} \mathrm{s}$ \\
\hline $\begin{array}{l}\text { Concen- } \\
\text { tration }\end{array}$ & molar & $\mathrm{nol} \mathrm{l}^{-1}=10^{3} \mathrm{~mol} \mathrm{~m}^{-3}$ \\
\hline Energy & joule & $\mathrm{J}=\mathrm{N} \mathrm{m}=\mathrm{kg} \mathrm{m}^{2} \mathrm{~s}^{-2}$ \\
\hline Force & newton & $\mathrm{N}=\mathrm{kg} \mathrm{m} \mathrm{s}^{-2}$ \\
\hline Frequency & hertz & $\mathrm{Hz}=\mathrm{s}^{-1}$ \\
\hline Power & watt & $\mathrm{W}=\mathrm{J} \mathrm{s}^{-1}=\mathrm{kg} \mathrm{m}^{2} \mathrm{~s}^{-3}$ \\
\hline Pressure & pascal & $=\mathrm{N} \mathrm{m}^{-2}=\mathrm{kg} \mathrm{m}^{-1} \mathrm{~s}^{-2}$ \\
\hline
\end{tabular}

$\begin{array}{lll}\text { ohm } & \Omega=\mathrm{V} \mathrm{A}^{-1}=\mathrm{kg} \mathrm{m}^{2} \mathrm{~A}^{-2} \mathrm{~s}^{-3} \\ \text { Voltage } & \text { volt } & \mathrm{V}=\mathrm{J} \mathrm{C}^{-1}=\mathrm{kg} \mathrm{m}^{2} \mathrm{~A}^{-1} \mathrm{~s}^{-3}\end{array}$

\section{REFERENCES}

Abendrot, R. P., 1970, J. Colloid Interface Sci. 34, 591.

Abgrall, P., L.-N. Low, and N.-T. Nguyen, 2007, Lab Chip 7, 520.

Adamczyk, Z., M. Zembala, P. Warszynski, and B. Jachimska, 2004, Langmuir 20, 10517.

Ahn, J.-H., H.-S. Kim, K. J. Lee, S. Jeon, S. J. Kang, Y. Sun, R. G. Nuzzo, and J. A. Rogers, 2006, Science 314, 1754.

Ajdari, A., and J. Prost, 1992, C. R. Acad. Sci., Ser. II: Mec., Phys., Chim., Sci. Terre Univers 315, 1635.

Aksimentiev, A., J. B. Heng, G. Timp, and K. Schulten, 2004, Biophys. J. 87, 2086.

Alem, H., F. Blondeau, K. Glinel, S. Demoustier-Champagne, and A. M. Jonas, 2007, Macromolecules 40, 3366.

Alexov, E., 2004, Eur. J. Biochem. 271, 173.

Anderson, N. L., and N. G. Anderson, 1998, Electrophoresis 19, 1853.

Asbury, C. L., and G. v. d. Engh, 1998, Biophys. J. 74, 1024.

Ashkenasy, N., J. Sanchez-Quesada, H. Bayley, and M. R. Ghadiri, 2005, Angew. Chem., Int. Ed. 44, 1401.

Austin, R. H., N. Darnton, R. Huang, J. Sturm, O. Bakajin, and T. Duke, 2002, Appl. Phys. A: Mater. Sci. Process. 75, 279.

Baldessari, F., and J. G. Santiago, 2006, J. Nanobiotechnol. 4, 12.1.

Balducci, A., P. Mao, J. Han, and P. S. Doyle, 2006, Macromolecules 39, 6273.

Basu, S., and M. M. Sharma, 1997, J. Membr. Sci. 124, 77.

Bates, M., M. Burns, and A. Meller, 2003, Biophys. J. 84, 2366.

Bath, B., H. White, and E. Scott, 2000, Anal. Chem. 72, 433.

Bayley, H., and P. S. Cremer, 2001, Nature 413, 226.

Bazant, M. Z., K. Thornton, and A. Ajdari, 2004, Phys. Rev. E 70, 021506.

Bean, C. P., M. V. Doyle, and G. Entine, 1970, J. Appl. Phys. 41, 1454.

Beck, R. E., and J. S. Schultz, 1970, Science 170, 1302.

Beckman, R., E. Johnston-Halperin, Y. Luo, J. E. Green, and J. R. Heath, 2005, Science 310, 465.

Beckstein, O., P. C. Biggin, P. Bond, J. N. Bright, C. Domene, A. Grottesi, J. Holyoake, and M. S. Sansom, 2003, FEBS Lett. 555, 85 .

Behrens, S. H., and M. Borkovec, 1999, Phys. Rev. E 60, 7040.

Behrens, S. H., and D. G. Grier, 2001, J. Chem. Phys. 115, 6716.

Ben, Y., and H.-C. Chang, 2002, J. Fluid Mech. 461, 229.

Bergveld, P., 1972, IEEE Trans. Biomed. Eng. 19, 342.

Berthold, A., L. Nicola, P. M. Sarro, and M. J. Vellekoop, 2000, Sens. Actuators, A 82, 224.

Bezrukov, S. M., and M. Winterhalter, 2000, Phys. Rev. Lett. 85, 202.

Bhatt, K., O. Velev, and S. Grego, 2005, Langmuir 21, 6603.

Bhattacharjee, S., J. Chen, and M. Elimelech, 2001, AIChE J. 47, 2733.

Bianchi, F., F. Wagner, P. Hoffmann, and H. H. Girault, 2001, Anal. Chem. 73, 829.

Bjork, M. T., C. Thelander, A. E. Hansen, L. E. Jensen, M. W. Larsson, L. R. Wallenberg, and L. Samuelson, 2004, Nano Lett. 4, 1621.

Bluhm, E. A., E. Bauer, R. M. Chamberlin, K. D. Abney, J. S. 
Young, and G. D. Jarvinen, 1999, Langmuir 15, 8668.

Bockris, J. O., and A. K. N. Reddy, 2000, Modern Electrochemistry, 2nd ed. (Kluwer Academic, New York), Vol. 2B.

Bonthuis, D. J., J. Zhang, B. Hornblower, J. Mathe, B. I. Shklovskii, and A. Meller, 2006, Phys. Rev. Lett. 97, 128104.

Bostrom, M., F. Tavares, S. Finet, F. Skouri-Panet, A. Tardieu, and B. Ninham, 2005, Biophys. Chem. 117, 217.

Bourlon, B., J. Wong, C. Miko, L. Forro, and M. Bockrath, 2007, Nat. Nanotechnol. 2, 104.

Bowen, R. W., W. A. Mohammad, and N. Hilal, 1997, J. Membr. Sci. 126, 91.

Bowen, W. R., and J. S. Welfoot, 2002, Chem. Eng. Sci. 57, 1121.

Burgreen, D., and F. Nakache, 1964, J. Phys. Chem. 68, 1084. Burns, D., and A. Zydney, 1999, Biotechnol. Bioeng. 64, 27.

Bushong, N., J. Gamble, and M. Di Ventra, 2007, Nano Lett. 7, 1789.

Cabodi, M., Y.-F. Chen, S. W. Turner, H. G. Craighead, and R. H. Austin, 2002, Electrophoresis 23, 3496.

Cabodi, M., S. W. Turner, and H. G. Craighead, 2002, Anal. Chem. 74, 5169.

Campbell, L. C., M. J. Wilkinson, A. Manz, P. Camilleri, and C. J. Humphreys, 2004, Lab Chip 4, 225.

Cannon, D. M., B. R. Flachsbart, M. A. Shannon, J. V. Sweedler, and P. W. Bohn, 2004, Appl. Phys. Lett. 85, 1241.

Cannon, D. M., T.-C. Kuo, P. W. Bohn, and J. V. Sweedler, 2003, Anal. Chem. 75, 224.

Cao, H., J. Tegenfeldt, R. Austin, and S. Y. Chou, 2002, Appl. Phys. Lett. 81, 3058.

Carbonaro, A., and L. L. Sohn, 2005, Lab Chip 5, 1155.

Carlen, E. T., and A. Van den Berg, 2007, Lab Chip 7, 19.

Cervera, J., A. Alcaraz, B. Schiedt, R. Neumann, and P. Ramirez, 2007, J. Phys. Chem. C 111, 12265.

Cervera, J., B. Schiedt, R. Neumann, S. Mafe, and P. Ramirez, 2006, J. Chem. Phys. 124, 104706.

Cevc, G., 1990, Biochim. Biophys. Acta 1031, 311.

Chan, E. Y., 2005, Mutat Res. 573, 13.

Chang, H., F. Kosari, G. Andreadakis, M. A. Alam, G. Vasmatzis, and R. Bashir, 2004, Nano Lett. 4, 1551.

Chaufer, B., M. Rabiller-Baudry, L. Guihard, and G. Daufin, 1996, Desalination 104, 37.

Chen, L., G. Luo, K. Liu, J. Ma, B. Yao, Y. Yan, and Y. Wang, 2006, Sens. Actuators B 119, 335.

Chen, P., J. Gu, E. Brandin, Y.-R. Kim, Q. Wang, and D. Branton, 2004, Nano Lett. 4, 2293.

Chen, P., T. Mitsui, D. B. Farmer, J. A. Golovchenko, R. G. Gordon, and D. Branton, 2004, Nano Lett. 4, 1333.

Chen, R. J., S. Bangsaruntip, K. A. Drouvalakis, N. W. S. Kam, M. Shim, Y. M. Li, W. Kim, P. J. Utz, and H. J. Dai, 2003, Proc. Natl. Acad. Sci. U.S.A. 100, 4984.

Chen, R. J., H. C. Choi, S. Bangsaruntip, E. Yenilmez, X. W. Tang, Q. Wang, Y. L. Chang, and H. J. Dai, 2004, J. Am. Chem. Soc. 126, 1563.

Cheng, J. T., and N. Giordano, 2002, Phys. Rev. E 65, 031206. Cheng, L. J., and L. J. Guo, 2007, Nano Lett. 7, 3165.

Cheng, X., and L. Guo, 2004, Microelectron. Eng. 71, 288.

Chou, C.-F., R. H. Austin, O. Bakajin, J. O. Tegenfeldt, J. A. Castelino, S. S. Chan, E. C. Cox, H. G. Craighead, N. Darnton, T. Duke, J. Han, and S. W. Turner, 2000, Electrophoresis 21, 81.

Chou, C. F., O. Bakajin, S. W. P. Turner, T. A. J. Duke, S. S. Chan, E. C. Cox, H. G. Craighead, and R. H. Austin, 1999, Proc. Natl. Acad. Sci. U.S.A. 96, 13762.
Chou, S. Y., and P. R. Krauss, 1997, Microelectron. Eng. 35, 237.

Chu, W.-H., R. Chin, T. Huen, and M. Ferrari, 1999, J. Microelectromech. Syst. 8, 34.

Chun, K.-Y., S. Mafe, P. Ramirez, and P. Stroeve, 2006, Chem. Phys. Lett. 418, 557.

Chun, K. Y., and P. Stroeve, 2002, Langmuir 18, 4653.

Collins, P. G., K. Bradley, M. Ishigami, and A. Zettl, 2000, Science 287, 1801.

Conlisk, A. T., 2005, Electrophoresis 26, 1896.

Conlisk, A. T., J. McFerran, Z. Zheng, and D. Hansford, 2002, Anal. Chem. 74, 2139.

Corthals, G. L., M. P. Molloy, B. R. Herbert, K. L. Williams, and A. A. Gooley, 1997, Electrophoresis 18, 317.

Craig Venter, J., et al., 2001, Science 291, 1304.

Craighead, H., 2006, Nature 442, 387.

Craighead, H. G., 2003, J. Vac. Sci. Technol. A 21, S216.

Cui, B., Z. Yu, H. Ge, and S. Y. Chou, 2007, Appl. Phys. Lett. 90, 043118.

Cui, Y., Q. Q. Wei, H. K. Park, and C. M. Lieber, 2001, Science 293, 1289.

Cui, Y., Z. H. Zhong, D. L. Wang, W. U. Wang, and C. M. Lieber, 2003, Nano Lett. 3, 149.

Cussler, E. L., 1997, Diffusion, Mass Transfer in Fluid Systems, 2nd ed. (Cambridge University, New York).

Daiguji, H., Y. Oka, and K. Shirono, 2005, Nano Lett. 5, 2274. Daiguji, H., P. Yang, and A. Majumdar, 2004, Nano Lett. 4, 137.

Daiguji, H., P. Yang, A. J. Szeri, and A. Majumdar, 2004, Nano Lett. 4, 2315.

Danelon, C., C. Santschi, J. Brugger, and H. Vogel, 2006, Langmuir 22, 10711.

Davis, J. A., R. O. James, and J. O. Leckie, 1978, J. Colloid Interface Sci. 63, 480.

De Franceschi, S., J. A. van Dam, E. Bakkers, L. F. Feiner, L. Gurevich, and L. P. Kouwenhoven, 2003, Appl. Phys. Lett. 83, 344.

De Leebeeck, A., and D. Sinton, 2006, Electrophoresis 27, 4999.

Deamer, D. W., and M. Akeson, 2000, Trends Biotechnol. 18, 147.

DeBlois, R. W., C. P. Bean, and R. K. A. Wesley, 1976, J. Colloid Interface Sci. 61, 323.

Decher, G., and J. B. Schlenoff, 2002, Multilayer Thin Films (Wiley-VCH, Weinheim, Germany).

Deen, W., 1987, AIChE J. 33, 1409.

de Gennes, P., 1971, J. Chem. Phys. 55, 572.

de Gennes, P., 1979, Scaling Concepts of Polymer Physics (Cornell University Press, Ithaca, NY).

de Jong, J., R. G. H. Lammertink, and M. Wessling, 2006, Lab Chip 6, 1125 .

Dekker, C., 1999, Phys. Today 52 (5), 22.

Dekker, C., 2007, Nat. Nanotechnol. 2, 209.

Delft, K. M. v., J. C. T. Eijkel, D. Mijatovic, T. S. Druzhinina, H. Rathgen, N. R. Tas, A. v. d. Berg, and F. Mugele, 2007, Nano Lett. 7, 345.

DeRosa, J. F., and R. B. Beard, 1977, IEEE Trans. Biomed. Eng. 24, 260.

Desai, T., D. Hansford, L. Kulinsky, A. Nashat, G. Rasi, J. Tu, Y. Wang, M. Zhang, and M. Ferrari, 1999, Biomed. Microdevices 2, 11.

Devreux, F., P. Barboux, M. Filoche, and B. Sapoval, 2001, J. Mater. Sci. 36, 1331. 
Di Ventra, M., S. Evoy, and J. R. Heflin, 2004, Introduction to Nanoscale Science and Technology, Nanostructure Science and Technology (Kluwer Academic, Boston).

Dilmore, M. F., D. E. Clark, and L. L. Hench, 1979, Ceram. Bull. 58, 1111.

Dittrich, P. S., K. Tachikawa, and A. Manz, 2006, Anal. Chem. 78, 3887.

Donnan, F. G., 1924, Chem. Rev. (Washington, D.C.) 1, 73.

Donnan, F. G., 1995, J. Membr. Sci. 100, 45.

Doremus, R. H., 1994, Glass Science, 2nd ed. (Wiley, New York).

Doyle, D., R. Pfuetzner, A. Kuo, R. MacKinnon, J. Cabral, J. Gulbis, S. Cohen, and B. Chait, 1998, Science 89, 244501.

Drazer, G., J. Koplik, A. Acrivos, and B. Khusid, 2002, Phys. Rev. Lett. 89, 244501.

Duke, T., and R. Austin, 1998, Phys. Rev. Lett. 80, 1552.

Dukhin, S. S., 1991, Adv. Colloid Interface Sci. 35, 173.

Dukhin, S. S., and V. Shilov, 1969, Colloid J. USSR 31, 564.

Duong-Hong, D., J.-S. Wang, G. R. Liu, Y. Z. Chen, J. Han, and G. Hadjiconstantinou, Nicolas, 2007, Microfluid. Nanofluid. 4, 219.

Dutta, D., and J. M. Ramsey, 2004, in Proceedings of Micro$T A S$, edited by T. Laurell, J. Nilsson, K. Jensen, D. J. Harrison, and J. P. Kutter (The Royal Society of Chemistry, Malmo, Sweden), Vol. 2, pp. 142-144.

Eijkel, J. C. T., 2007, Lab Chip 7, 299.

Eijkel, J. C. T., and A. van den Berg, 2005a, Microfluid. Nanofluid. 1, 249.

Eijkel, J. C. T., and A. van den Berg, 2005b, Lab Chip 5, 1202. Eijkel, J. C. T., and A. van den Berg, 2006, Lab Chip 6, 19.

Elam, J. W., D. Routkevitch, P. P. Mardilovich, and S. M. George, 2003, Chem. Mater. 15, 3507.

Elton, G., 1948, Proc. R. Soc. London, Ser. A 194, 259.

Erickson, D., D. Q. Li, and C. Werner, 2000, J. Colloid Interface Sci. 232, 186.

Ertas, D., 1998, Phys. Rev. Lett. 80, 1548.

Ervin, E. N., H. S. White, and L. A. Baker, 2005, Anal. Chem. 77, 5564.

Ervin, E. N., H. S. White, L. A. Baker, and C. R. Martin, 2006, Anal. Chem. 78, 6535.

Fair, J., and J. Osterle, 1970, J. Chem. Phys. 54, 3307.

Fan, H. J., P. Werner, and M. Zacharias, 2006, Small 2, 700.

Fan, R., R. Karnik, M. Yue, D. Li, A. Majumdar, and P. Yang, 2005, Nano Lett. 5, 1633.

Farmer, D., and R. Gordon, 2006, Nano Lett. 6, 699.

Fawcett, W. R., and T. G. Smagala, 2006, Langmuir 22, 10635. Ferry, J. D., 1936, J. Gen. Physiol. 20, 95.

Flachsbart, B. R., K. Wong, J. M. Iannacone, E. N. Abante, R. L. Vlach, P. A. Rauchfuss, P. W. Bohn, J. V. Sweedler, and M. A. Shannon, 2006, Lab Chip 6, 667.

Fologea, D., M. Gershow, B. Ledden, D. S. McNabb, J. Golovchenko, and J. Li, 2005, Nano Lett. 5, 1905.

Fologea, D., J. Uplinger, B. Thomas, D. S. McNabb, and J. Li, 2005, Nano Lett. 5, 1734.

Foote, R. S., J. Khandurina, S. C. Jacobson, and J. M. Ramsey, 2005, Anal. Chem. 77, 57.

Foquet, M., J. Korlach, W. Zipfel, W. W. Webb, and H. G. Craighead, 2002, Anal. Chem. 74, 1415.

Foquet, M., J. Korlach, W. Zipfel, W. W. Webb, and H. G. Craighead, 2004, Anal. Chem. 76, 1618.

Foriers, A., E. Lebrun, R. Van Rapenbusch, R. de Neve, and A. Strosberg, 1981, J. Biol. Chem. 256, 5550.

Freire, S. L. S., and A. R. Wheeler, 2006, Lab Chip 6, 1415.
Fu, J., P. Mao, and J. Han, 2005, Appl. Phys. Lett. 87, 263902. Fu, J., R. B. Schoch, A. L. Stevens, S. R. Tannenbaum, and J. Han, 2007, Nat. Nanotechnol. 2, 121.

Fu, J., J. Yoo, and J. Han, 2006, Phys. Rev. Lett. 97, 018103.

Fulinski, A., I. D. Kosinska, and Z. Siwy, 2004, Europhys. Lett. 67, 683.

Futterer, C., N. Minc, V. Bormuth, J. H. Codarbox, P. Laval, J. Rossier, and J. L. Viovy, 2004, Lab Chip 4, 351.

Gamby, J., J.-P. Abid, M. Abid, J.-P. Ansermet, and H. Girault, 2006, Anal. Chem. 78, 5289.

Garba, Y., S. Taha, N. Gondrexon, and G. Dorange, 1999, J. Membr. Sci. 160, 187.

Garcia, A. L., L. K. Ista, D. N. Petsev, M. J. O’Brien, P. Bisong, A. A. Mammoli, S. R. J. Brueck, and G. P. Lopez, 2005, Lab Chip 5, 1271.

Garcia-Aleman, J., and J. Dickson, 2004, J. Membr. Sci. 235, 1. Gasparac, R., D. T. Mitchell, and C. R. Martin, 2003, Electrochim. Acta 49, 847.

Gauthier, M., and G. Slater, 2003, Electrophoresis 24, 441.

Gawad, S., 2004, Ph.D. thesis (EPFL).

Ghosal, S., 2007, Phys. Rev. Lett. 98, 238104.

Ghowsi, K., and R. J. Gale, 1991, J. Chromatogr. 559, 95.

Giddings, J. C., 1965, Dynamics of Chromatography. Part 1:

Principles and Theory (Dekker, New York).

Giddings, J. C., 1991, Unified Separation Science (Wiley, New York).

Giddings, J. C., E. Kucera, C. P. Russell, and M. N. Myers, 1968, J. Phys. Chem. 72, 4397.

Giovine, E., A. Notargiacomo, L. Di Gaspare, E. Palange, F. Evangelisti, R. Leoni, G. Castellano, G. Torrioli, and V. Foglietti, 2001, Nanotechnology 12, 132.

Girault, H. H., 2004, Analytical and Physical Electrochemistry (Dekker, Basel).

Gohda, Y., and S. T. Pantelides, 2005, Nano Lett. 5, 1217.

Gooding, J. J., 2006, Small 2, 313.

Gracheva, M. E., J. Vidal, and J.-P. Leburton, 2007, Nano Lett. 7, 1717.

Gracheva, M. E., A. L. Xiong, A. Aksimentiev, K. Schulten, G. Timp, and J. P. Leburton, 2006, Nanotechnology 17, 622.

Graul, T. W., and J. B. Schlenoff, 1999, Anal. Chem. 71, 4007. Gray, J., 2004, Curr. Opin. Struct. Biol. 14, 110.

Griffiths, S. K., and R. H. Nilson, 1999, Anal. Chem. 71, 5522. Griffiths, S. K., and R. H. Nilson, 2006, Anal. Chem. 78, 8134. Groot, R. D., and P. B. Warren, 1997, J. Chem. Phys. 107, 4423. Gross, R., and J. Osterle, 1968, J. Chem. Phys. 49, 228.

Gu, Y., and D. Li, 2000, J. Colloid Interface Sci. 226, 328. Guo, L. J., X. Cheng, and C.-F. Chou, 2003, Nano Lett. 4, 69. Gygi, S. P., G. L. Corthals, Y. Zhang, Y. Rochon, and R. Aebersold, 2000, Proc. Natl. Acad. Sci. U.S.A. 97, 9390.

Haber, C., 2006, Lab Chip 6, 1118.

Hagmeyer, G., and R. Gimbel, 1999, Sep. Purif. Technol. 15, 19.

Hahm, J.-I., and C. Lieber, 2004, Nano Lett. 4, 51.

Hamann, C. H., A. Hamnett, and W. Vielstich, 1998, Electrochemistry (Wiley-VCH, Weinheim, Germany).

Han, A., G. Mondin, N. G. Hegelbach, N. F. de Rooij, and U. Staufer, 2006, J. Colloid Interface Sci. 293, 151.

Han, A., G. Schurmann, G. Mondin, R. A. Bitterlil, N. G. Hegelbach, N. F. De Rooij, and U. Staufer, 2006, Appl. Phys. Lett. 88, 093901.

Han, A. P., N. F. de Rooij, and U. Staufer, 2006, Nanotechnology 17, 2498.

Han, J., 2004, in Introduction to Nanoscale Science and Tech- 
nology, edited by M. Di Ventra, S. Evoy, and J. R. Heflin (Kluwer Academic, Boston).

Han, J., and H. Craighead, 2002, Anal. Chem. 74, 394.

Han, J., and H. G. Craighead, 1999, J. Vac. Sci. Technol. A 17, 2142.

Han, J., and H. G. Craighead, 2000, Science 288, 1026.

Han, J., S. W. Turner, and H. G. Craighead, 1999, Phys. Rev. Lett. 83, 1688.

Haneveld, J., H. Jansen, E. Berenschot, N. Tas, and M. Elwenspoek, 2003, J. Micromech. Microeng. 13, S62.

Harrell, C. C., Y. Choi, L. P. Horne, L. A. Baker, Z. S. Siwy, and C. R. Martin, 2006, Langmuir 22, 10837.

Harrell, C. C., P. Kohli, Z. Siwy, and C. R. Martin, 2004, J. Am. Chem. Soc. 126, 15646.

Harrell, C. C., S. B. Lee, and C. R. Martin, 2003, Anal. Chem. 75, 6861.

Hatch, A. V., A. E. Herr, D. J. Throckmorton, J. S. Brennan, and A. K. Sing, 2006, Anal. Chem. 78, 4976.

Hawkins Cwirko, E., and R. G. Carbonell, 1989, J. Colloid Interface Sci. 129, 513.

He, B., N. Tait, and F. Regnier, 1998, Anal. Chem. 70, 3790.

Healy, T. W., D. Chan, and L. R. White, 1980, Pure Appl. Chem. 52, 1207.

Heinze, S., J. Tersoff, R. Martel, V. Derycke, J. Appenzeller, and P. Avouris, 2002, Phys. Rev. Lett. 89, 106801.

Helfferich, F., 1962, Ion Exchange (McGraw-Hill, New York). Hench, L. L., and D. E. Clark, 1978, J. Non-Cryst. Solids 28, 83.

Heng, J. B., A. Aksimentiev, C. Ho, P. Marks, Y. V. Grinkova, S. Sligar, K. Schulten, and G. Timp, 2005, Nano Lett. 5, 1883.

Heng, J. B., A. Aksimentiev, C. Ho, P. Marks, Y. V. Grinkova, S. Sligar, K. Schulten, and G. Timp, 2006, Biophys. J. 90, 1098.

Heng, J. B., C. Ho, T. Kim, R. Timp, A. Aksimentiev, Y. V. Grinkova, S. Sligar, K. Schulten, and G. Timp, 2004, Biophys. J. 87, 2905.

Hess, S. T., S. H. Huang, A. A. Heikal, and W. W. Webb, 2002, Biochemistry 41, 697.

Heyderman, L. J., B. Ketterer, D. Bachle, F. Glaus, B. Haas, H. Schift, K. Vogelsang, J. Gobrecht, L. Tiefenauer, and O. Dubochet, 2003, Microelectron. Eng. 67-68, 208.

Ho, C., R. Qiao, J. B. Heng, A. Chatterjee, R. J. Timp, N. R. Aluru, and G. Timp, 2005, Proc. Natl. Acad. Sci. U.S.A. 102, 10445.

Hollman, A. M., and D. Bhattacharyya, 2004, Langmuir 20, 5418.

Holt, J. K., H. G. Park, Y. M. Wang, M. Stadermann, A. B. Artyukhin, C. P. Grigoropoulos, A. Noy, and O. Bakajin, 2006, Science 312, 1034.

Holtzel, A., and U. Tallarek, 2007, J. Sep. Sci. 30, 1398.

Horn, J. M., and G. Y. Onoda, 1978, J. Am. Ceram. Soc. 61, 523.

Hornblower, B., A. Coombs, R. D. Whitaker, A. Kolomeisky, S. J. Picone, A. Meller, and M. Akeson, 2007, Nat. Methods 4, 315.

Howorka, S., and H. Bayley, 2002, Biophys. J. 83, 3202.

Howorka, S., S. Cheley, and H. Bayley, 2001, Nat. Biotechnol. 19, 636.

Huang, L., J. Tegenfeld, J. Kraeft, J. Sturm, R. Austin, and E. Cox, 2002, Nat. Biotechnol. 20, 1048.

Huang, L. R., E. C. Cox, R. H. Austin, and J. C. Sturm, 2004, Science 304, 987.

Huang, L. R., P. Silberzan, J. O. Tegenfeldt, E. C. Cox, J. C.
Sturm, R. H. Austin, and H. Craighead, 2002, Phys. Rev. Lett. 89, 178301.

Huang, N. P., J. Voros, S. M. De Paul, M. Textor, and N. D. Spencer, 2002, Langmuir 18, 220.

Huang, Y., X. F. Duan, Q. Q. Wei, and C. M. Lieber, 2001, Science 291, 630.

Huebsch, E., V. Ball, B. Senger, G. Decher, J. C. Voegel, and P. Schaaf, 2004, Langmuir 20, 1980.

Hughes, M. P., 2000, Nanotechnology 11, 124.

Hunter, R. J., 1981, Zeta Potential in Colloid Science (Academic, London).

Hunter, R. J., and H. J. L. Wright, 1971, J. Colloid Interface Sci. 37, 564.

Iler, R. K., 1979, The Chemistry of Silica (Wiley, New York).

Ionescu-Zanetti, C., J. T. Nevill, D. Di Carlo, K. H. Jeong, and L. P. Lee, 2006, J. Appl. Phys. 99, 024305.

Iqbal, S. M., D. Akin, and R. Bashir, 2007, Nat. Nanotechnol. 2, 243.

Israelachvili, J., 1992, Intermolecular and Surface Forces, 2nd ed. (Academic, London).

Ito, T., K. Sobue, and S. Ohya, 2002, Sens. Actuators B 81, 187. Ito, T., L. Sun, and R. M. Crooks, 2003, Anal. Chem. 75, 2399. Javey, A., S. Nam, R. S. Friedman, H. Yan, and C. M. Lieber, 2007, Nano Lett. 7, 773.

Jednacak, J., and V. Pravdic, 1974, J. Colloid Interface Sci. 49, 16.

Jimbo, T., P. Ramirez, A. Tanioka, S. Mafe, and N. Minoura, 2000, J. Colloid Interface Sci. 225, 447.

Jin, S., D. M. Whang, M. C. McAlpine, R. S. Friedman, Y. Wu, and C. M. Lieber, 2004, Nano Lett. 4, 915.

Jo, K., D. M. Dhingra, T. Odijk, J. J. de Pablo, M. D. Graham, R. Runnheim, D. Forrest, and D. C. Schwartz, 2007, Proc. Natl. Acad. Sci. U.S.A. 104, 2673.

Johann, R., and P. Renaud, 2004, Electrophoresis 25, 3720.

Jones, A., and P. Chalker, 2003, J. Phys. D 36, R80.

Kaji, N., Y. Tezuka, Y. Takamura, M. Ueda, T. Nishimoto, H. Nakanishi, Y. Horiike, and Y. Baba, 2004, Anal. Chem. 76, 15. Karhanek, M., J. T. Kemp, N. Pourmand, R. W. Davis, and C. D. Webb, 2005, Nano Lett. 5, 403.

Karnik, R., K. Castelino, C. Duan, and A. Majumdar, 2006, Nano Lett. 6, 1735.

Karnik, R., K. Castelino, R. Fan, P. Yang, and A. Majumdar, 2005, Nano Lett. 5, 1638.

Karnik, R., K. Castelino, and A. Majumdar, 2006, Appl. Phys. Lett. 88, 123114.

Karnik, R., C. Duan, K. Castelino, H. Daiguji, and A. Majumdar, 2007, Nano Lett. 7, 547.

Karnik, R., R. Fan, M. Yue, D. Li, P. Yang, and A. Majumdar, 2005, Nano Lett. 5, 943.

Kasianowicz, J. J., E. Brandin, D. Branton, and D. W. Deamer, 1996, Proc. Natl. Acad. Sci. U.S.A. 93, 13770.

Kemery, P. J., J. K. Steehler, and P. W. Bohn, 1998, Langmuir 14, 2884.

Kenausis, G. L., J. Voros, D. L. Elbert, N. P. Huang, R. Hofer, L. Ruiz-Taylor, M. Textor, J. A. Hubbell, and N. D. Spencer, 2000, J. Phys. Chem. B 104, 3298.

Keyser, U. F., B. N. Koeleman, S. Van Dorp, D. Krapf, R. M. M. Smeets, S. G. Lemay, N. H. Dekker, and C. Dekker, 2006, Nat. Phys. 2, 473.

Keyser, U. F., J. van der Does, C. Dekker, and N. H. Dekker, 2006, Rev. Sci. Instrum. 77, 105105.

Khandurina, J., S. C. Jacobson, L. C. Waters, R. S. Foote, and J. M. Ramsey, 1999, Anal. Chem. 71, 1815. 
Kim, S. J., Y.-C. Wang, J. H. Lee, H. Jang, and J. Han, 2007 Phys. Rev. Lett. 99, 044501.

King, G. M., G. Schurmann, D. Branton, and J. Golovchenko, 2005, Nano Lett. 5, 1157.

Kirby, B. J., and E. F. Hasselbrink, 2004, Electrophoresis 25, 187.

Kittaka, S., and T. Morimoto, 1976, J. Colloid Interface Sci. 55, 431.

Kittilsland, G., G. Stemme, and B. Norden, 1990, Sens. Actuators, A 23, 904.

Knowles, K. M., and A. T. J. van Helvoort, 2006, Int. Mater. Rev. 51, 273.

Kobayashi, Y., and C. R. Martin, 1997, J. Electroanal. Chem. 431, 29.

Koch, M., A. G. R. Evans, and A. Brunnschweiler, 1999, J. Micromech. Microeng. 9, 159.

Koehne, J. E., H. Chen, A. M. Cassell, Q. Yi, J. Han, M. Meyyappan, and J. Li, 2004, Clin. Chem. 50, 1886.

Kovacs, G. T., 1994, in Enabling Technologies for Cultured Neural Networks, edited by D. A. Stenger and T. M. McKenna (Academic, San Diego).

Krapf, D., B. M. Quinn, M.-Y. Wu, H. W. Zandbergen, C. Dekker, and S. G. Lemay, 2006, Nano Lett. 6, 2531.

Krapf, D., M. Y. Wu, R. M. M. Smeets, H. W. Zandbergen, C.

Dekker, and S. G. Lemay, 2006, Nano Lett. 6, 105.

Krasemann, L., and B. Tieke, 2000, Langmuir 16, 287.

Krishnan, M., I. Monch, and P. Schwille, 2007, Nano Lett. 7, 1270.

Ku, J.-R., and P. Stroeve, 2004, Langmuir 20, 2030.

Kuo, T.-C., D. M. Cannon, Y. Chen, J. J. Tulock, M. A. Shannon, J. V. Sweedler, and P. W. Bohn, 2003, Anal. Chem. 75, 1861.

Kwak, H. S., and E. F. Hasselbrink, 2005, J. Colloid Interface Sci. 284, 753.

Lagerqvist, J., M. Zwolak, and M. Di Ventra, 2006, Nano Lett. 6, 779 .

Lambert, J.-P., M. Ethier, J. C. Smith, and D. Figeys, 2005, Anal. Chem. 77, 3771.

Lander, E., et al., 2001, Nature 409, 860.

Lauga, E., M. P. Brenner, and H. A. Stone, 2006, in Handbook of Experimental Fluid Dynamics, edited by J. Foss, C. Tropea, and A. Yarin (Springer, New York).

Layson, A., and D. Teeters, 2004, Solid State Ionics 175, 773.

Lee, C., E.-H. Yang, N. V. Myung, and T. George, 2003, Nano Lett. 3, 1339.

Lee, D., A. J. Nolte, A. L. Kunz, M. F. Rubner, and R. E. Cohen, 2006, J. Am. Chem. Soc. 128, 8521.

Lee, D.-J., Y.-H. Lee, J. Jang, and B.-K. Ju, 2001, Sens. Actuators, A 89, 43.

Lee, S. B., and C. R. Martin, 2001, Anal. Chem. 73, 768.

Leinweber, F. C., M. Pfafferodt, A. Seidel-Morgenstern, and U. Tallarek, 2005, Anal. Chem. 77, 5839.

Leinweber, F. C., and U. Tallarek, 2004, Langmuir 20, 11637.

Levich, V. G., 1962, Physical Hydrodynamics (Prentice-Hall, London).

Levine, S., J. Marriott, G. Neale, and N. Epstein, 1975, J. Colloid Interface Sci. 52, 136.

Levine, S., J. R. Marriott, and K. Robinson, 1975, J. Chem. Soc., Faraday Trans. 2 71, 1.

Li, B. Q., X. H. Fang, H. B. Luo, Y. S. Seo, E. Petersen, Y. Ji, M. Rafailovich, J. Sokolov, D. Gersappe, and B. Chu, 2006, Anal. Chem. 78, 4743.

Li, J., D. Stein, C. McMullan, D. Branton, M. J. Aziz, and J. A.
Golovchenko, 2001, Nature 412, 166.

Li, J. L., M. Gershow, D. Stein, E. Brandin, and J. A. Golovchenko, 2003, Nat. Mater. 2, 611.

Li, N., S. Yu, C. C. Harrell, and C. R. Martin, 2004, Anal. Chem. 76, 2025.

Li, Z., Y. Chen, X. Li, T. I. Kamins, K. Nauka, and R. S. Williams, 2004, Nano Lett. 4, 245.

Li, Z., B. Rajendran, T. I. Kamins, X. Li, Y. Chen, and R. S. Williams, 2005, Appl. Phys. A: Mater. Sci. Process. 80, 1257.

Liang, G., J. Xiang, N. Kharche, G. Klimeck, C. M. Lieber, and M. Lundstrom, 2007, Nano Lett. 7, 642.

Lichtenberg, J., N. F. de Rooij, and E. Verpoorte, 2002, Talanta 56, 233.

Lieber, C. M., 2003, MRS Bull. 28, 486.

Lin, C., Y. Liu, and H. Yan, 2007, Nano Lett. 7, 507.

Lionello, A., J. Josserand, H. Jensen, and H. Girault, 2005, Lab Chip 5, 254.

Liu, H., S. Qian, and H. H. Bau, 2007, Biophys. J. 92, 1164.

Liu, S., Q. Pu, L. Gao, C. Korzeniewski, and C. Matzke, 2005, Nano Lett. 5, 1389.

Lobbus, M., J. Sonnfeld, H. P. van Leeuwen, W. Vogelsberger, and J. Lyklema, 2000, J. Colloid Interface Sci. 229, 174.

Lu, D., Y. Li, S. V. Rotkin, U. Ravaioli, and K. Schulten, 2004, Nano Lett. 4, 2383.

Lubensky, D. K., and D. R. Nelson, 1999, Biophys. J. 77, 1824.

Lulevich, V. V., and O. I. Vinogradova, 2004, Langmuir 20, 2874.

Lund, M., T. Akesson, and B. Jonsson, 2005, Langmuir 21, 8385.

Lyklema, J., 1995, Solid-liquid Interfaces, Vol. 2 of Fundamentals of Interface and Colloid Science (Academic, San Diego). Lyklema, J., 2001, J. Phys.: Condens. Matter 13, 5027.

Lyklema, J., and M. Minor, 1998, Colloids Surf., A 140, 33.

Lyklema, J., and J. T. Overbeek, 1961, J. Colloid Sci. 16, 501.

Lyklema, J., S. Rovillard, and J. De Coninck, 1998, Langmuir 14, 5659.

Ma, D. D. D., C. S. Lee, F. C. K. Au, S. Y. Tong, and S. T. Lee, 2003, Science 299, 1874.

MacDonald, J. R., 1987, Impedance Spectroscopy: Emphasizing Solid Materials and Systems (Wiley, New York).

Madou, M., 1997, Fundamentals of Microfabrication (CRC, New York).

Mafe, S., and P. Ramirez, 1997, Acta Polym. 48, 234.

Magde, D., E. Elson, and W. W. Webb, 1972, Phys. Rev. Lett. 29, 705.

Majumder, M., N. Chopra, R. Andrews, and B. J. Hinds, 2005, Nature 438, 44.

Maletzki, F., H. W. Rosler, and E. Staude, 1992, J. Membr. Sci. 71, 105.

Malone, D. M., and J. L. Anderson, 1978, Chem. Eng. Sci. 33, 1429.

Manning, G., 1978, Q. Rev. Biophys. 11, 179.

Mannion, J. T., C. H. Reccius, J. D. Cross, and H. G. Craighead, 2006, Biophys. J. 90, 4538.

Manz, A., N. Graber, and H. M. Widmer, 1990, Sens. Actuators B 1, 244.

Mao, P., and J. Han, 2005, Lab Chip 5, 837.

Mara, A., Z. Siwy, C. Trautmann, J. Wan, and F. Kamme, 2004, Nano Lett. 4, 497.

Marie, R., J. P. Beech, J. Voros, J. O. Tegenfeldt, and F. Hook, 2006, Langmuir 22, 10103.

Marshall, G., and P. Mocskos, 1997, Phys. Rev. E 55, 549.

Martin, C. R., M. Nishizawa, K. Jirage, M. S. Kang, and S. B. 
Lee, 2001, Adv. Mater. 13, 1351.

Martin, F. J., and C. Grove, 2001, Biomed. Microdevices 3, 97.

Mathe, J., H. Visram, V. Viasnoff, Y. Rabin, and A. Meller, 2004, Biophys. J. 87, 3205.

Matthias, S., and F. Muller, 2003, Nature 424, 53.

McAdams, E. T., A. Lackermeier, J. A. McLaughlin, D.

Macken, and J. Jossinet, 1995, Biosens. Bioelectron. 10, 67.

Meller, A., 2003, J. Phys.: Condens. Matter 15, 581.

Meller, A., L. Nivon, E. Brandin, J. Golovchenko, and D.

Branton, 2000, Proc. Natl. Acad. Sci. U.S.A. 97, 1079.

Meller, A., L. Nivon, and D. Branton, 2001, Phys. Rev. Lett. 86, 3435 .

Menon, M., and A. Zydney, 2000, Anal. Chem. 72, 5714.

Metz, S., 2003, Ph.D. thesis (EPFL).

Mijatovic, D., J. C. T. Eijkel, and A. van den Berg, 2005, Lab Chip 5, 492.

Miller, S., V. Young, and C. Martin, 2001, J. Am. Chem. Soc. 123, 12335.

Min, J. Y., E. F. Hasselbrink, and S. J. Kim, 2004, Sens. Actuators B 98, 368.

Mishchuk, N. A., and P. V. Takhistov, 1995, Colloids Surf., A 95, 119.

Mockel, D., E. Staude, M. Dal-Cin, K. Darcovich, and M. Guiver, 1998, J. Membr. Sci. 145, 211.

Moorthy, J., C. Khoury, J. S. Moore, and D. J. Beebe, 2001, Sens. Actuators B 75, 223.

Morales, A. M., and C. M. Lieber, 1998, Science 279, 208.

Morgan, H., and N. G. Green, 2003, AC Electrokinetics: Colloids and Nanoparticles (Research Studies, Hertfordshire).

Morrison, F., and J. Osterle, 1965, J. Chem. Phys. 43, 2111.

Mukhopadhyay, R., 2006, Anal. Chem. 78, 7379.

Muthukumar, M., 2001, Phys. Rev. Lett. 86, 3188.

Muthukumar, M., 2003, J. Chem. Phys. 118, 5174.

Muthukumar, M., and A. Baumgartner, 1989, Macromolecules 22, 1937.

Muthukumar, M., and C. Y. Kong, 2006, Proc. Natl. Acad. Sci. U.S.A. 103, 5273.

Nabil, L., D. Carmelo, M. Christina, and D. D. Kevin, 2007, Phys. Rev. Lett. 98, 098106.

Nakanishi, H., T. Nishimoto, R. Nakamura, A. Yotsumoto, T. Yoshida, and S. Shoji, 2000, Sens. Actuators, A 79, 237.

Nakanishi, K., T. Sakiyama, and K. Imamura, 2001, J. Econ. Dyn. Control 91, 233.

Neto, C., D. R. Evans, E. Bonaccurso, H. J. Butt, and V. S. J. Craig, 2005, Rep. Prog. Phys. 68, 2859.

Niklaus, F., H. Andersson, P. Enoksson, and G. Stemme, 2001, Sens. Actuators, A 92, 235.

Niklaus, F., G. Stemme, J. Q. Lu, and R. J. Gutmann, 2006, J. Appl. Phys. 99, 031101.

Nilson, R. H., and S. K. Griffiths, 2006, J. Chem. Phys. 125, 164510.

Ninham, B. W., and V. A. Parsegian, 1971, J. Theor. Biol. 31, 405.

Nischang, I., G. Chen, and U. Tallarek, 2006, J. Chromatogr., A 1109, 32.

Nishizawa, M., V. Menon, and C. Martin, 1995, Science 268, 700 .

Norde, W., 1986, Adv. Colloid Interface Sci. 25, 267.

Oddy, M. H., and J. G. Santiago, 2004, J. Colloid Interface Sci. 269, 192.

Ogston, A. G., 1958, Trans. Faraday Soc. 54, 1754.

Okazaki, S., 1991, J. Vac. Sci. Technol. B 9, 2829.

Overbeek, J. T. G., 1952, in Colloid Science, edited by H. R.
Kruyt (Elsevier, Amsterdam), Vol. 1.

Pappenheimer, J. R., E. M. Renkin, and L. M. Borrero, 1951, Am. J. Physiol. 167, 13.

Park, S.-M., and J.-S. Yoo, 2003, Anal. Chem. 75, 455A.

Parks, G. A., 1965, Chem. Rev. (Washington, D.C.) 65, 177.

Patolsky, F., B. P. Timko, G. H. Yu, Y. Fang, A. B. Greytak, G.

F. Zheng, and C. M. Lieber, 2006, Science 313, 1100.

Patolsky, F., G. F. Zheng, O. Hayden, M. Lakadamyali, X. W. Zhuang, and C. M. Lieber, 2004, Proc. Natl. Acad. Sci. U.S.A. 101, 14017.

Patolsky, F., G. F. Zheng, and C. M. Lieber, 2006a, Anal. Chem. 78, 4260.

Patolsky, F., G. F. Zheng, and C. M. Lieber, 2006b, Nanomedicine $\mathbf{1}, 51$.

Peng, C.-Y., W. J. Nam, S. J. Fonash, B. Gu, A. Sen, K. Strawhecker, S. Natarajan, H. C. Foley, and S. H. Kim, 2003, J. Am. Chem. Soc. 125, 9298.

Pennathur, S., and J. G. Santiago, 2005a, Anal. Chem. 77, 6772. Pennathur, S., and J. G. Santiago, 2005b, Anal. Chem. 77, 6782. Perram, J. W., R. J. Hunter, and H. J. L. Wright, 1973, Chem. Phys. Lett. 23, 265.

Petersen, N. J., D. Dutta, J. P. Alarie, and J. M. Ramsey, 2004, in Proceedings of MicroTAS, edited by T. Laurell, J. Nilsson, K. Jensen, D. J. Harrison, and J. P. Kutter (The Royal Society of Chemistry, Malmo, Sweden), Vol. 1, pp. 348-350.

Plecis, A., R. B. Schoch, and P. Renaud, 2005, Nano Lett. 5, 1147.

Poptoshev, E., B. Schoeler, and F. Caruso, 2004, Langmuir 20, 829.

Pu, Q., J. Yun, H. Temkin, and S. Liu, 2004, Nano Lett. 4, 1099. Pujar, N., and A. Zydney, 1997, J. Colloid Interface Sci. 192, 338.

Qiao, R., and N. R. Aluru, 2003, J. Chem. Phys. 118, 4692.

Qiao, R., and N. R. Aluru, 2005, Langmuir 21, 8972.

Qu, W. L., and D. Q. Li, 2000, J. Colloid Interface Sci. 224, 397.

Quake, S. R., and A. Scherer, 2000, Science 290, 1536.

Rabilloud, T., 2002, Proteomics 2, 3.

Rabin, Y., and M. Tanaka, 2005, Phys. Rev. Lett. 94, 148103.

Ramachandran, N., D. N. Larson, P. R. H. Stark, E. Hainsworth, and J. LaBaer, 2005, FEBS J. 272, 5412.

Ramirez, P., S. Mafe, V. M. Aguilella, and A. Alcaraz, 2003, Phys. Rev. E 68, 011910.

Ramirez, P., S. Mafe, A. Alcaraz, and J. Cervera, 2003, J. Phys. Chem. B 107, 13178.

Reisner, W., K. J. Morton, R. Riehn, Y. M. Wang, Z. N. Yu, M. Rosen, J. C. Sturm, S. Y. Chou, E. Frey, and R. H. Austin, 2005, Phys. Rev. Lett. 94, 196101.

Ren, L., D. Li, and W. Qu, 2001, J. Colloid Interface Sci. 233, 12.

Renkin, E. M., 1954, J. Gen. Physiol. 38, 225.

Reza, S., G. Bosman, A. S. Islam, T. I. Kamins, S. Sharma, and R. S. Williams, 2006, IEEE Trans. Nanotechnol. 5, 523.

Rice, C. L., and R. Whitehead, 1965, J. Phys. Chem. 69, 4017. Richards, D. P., C. Stathakis, R. Polakowski, H. Ahmadzadeh, and N. J. Dovichi, 1999, J. Chromatogr., A 853, 21.

Rieger, P. H., 1994, Electrochemistry, 2nd ed. (Chapman and Hall, London).

Riehn, R., and R. Austin, 2006, Anal. Chem. 78, 5933.

Riehn, R., R. H. Austin, and J. C. Sturm, 2006, Nano Lett. 6, 1973.

Riehn, R., M. C. Lu, Y. M. Wang, S. F. Lim, E. C. Cox, and R. H. Austin, 2005, Proc. Natl. Acad. Sci. U.S.A. 102, 10012.

Righetti, P. G., C. Gelfi, and M. R. D'Acunto, 2002, Electro- 
phoresis 23, 1361.

Rodbard, D., and A. Chrambach, 1970, Proc. Natl. Acad. Sci. U.S.A. 65, 970.

Rousseau, J., G. Drouin, and G. W. Slater, 1997, Phys. Rev. Lett. 79, 1945.

Rubinshtein, I., B. Zaltzman, J. Pretz, and C. Linder, 2002, Russ. J. Electrochem. 38, 853.

Rubinstein, I., 1981, J. Chem. Soc., Faraday Trans. 2 77, 1595.

Rubinstein, I., 1995, Physical Electrochemistry (Dekker, New York).

Rubinstein, I., and B. Zaltzman, 2000, Phys. Rev. E 62, 2238.

Rubinstein, I., B. Zaltzman, and O. Kedem, 1997, J. Membr. Sci. 125, 17.

Saleh, O., and L. Sohn, 2001, Rev. Sci. Instrum. 72, 4449.

Saleh, O., and L. Sohn, 2002, Rev. Sci. Instrum. 73, 4396.

Saleh, O. A., and L. L. Sohn, 2003a, Nano Lett. 3, 37.

Saleh, O. A., and L. L. Sohn, 2003b, Proc. Natl. Acad. Sci. U.S.A. 100, 820.

Sasaki, M., M. Takagi, and Y. Okamura, 2006, Science 312, 589.

Sasidhar, V., and E. Ruckenstein, 1982, J. Colloid Interface Sci. 85, 332 .

Sata, T., 2004, Ion Exchange Membranes (Royal Society of Chemistry, Cambridge).

Satoh, A., 1999, Sens. Actuators, A 72, 160.

Sayah, A., D. Solignac, T. Cueni, and M. A. M. Gijs, 2000, Sens. Actuators, A 84, 103.

Schasfoort, R. B., S. Schlautmann, J. Hendrikse, and A. van den Berg, 1999, Science 286, 942.

Schellenberg, F. M., 2005, Opt. Rev. 12, 83.

Schiedt, B., K. Healy, A. P. Morrison, R. Neumann, and Z. Siwy, 2005, Nucl. Instrum. Methods Phys. Res. B 236, 109.

Schmid, G., 1998, J. Membr. Sci. 150, 151.

Schmidt, D., Q.-X. Jiang, and R. MacKinnon, 2006, Nature 444, 775.

Schmuhl, R., K. Keizer, A. van den Berg, J. E. ten Elshof, and D. H. A. Blank, 2004, J. Colloid Interface Sci. 273, 331.

Schmuhl, R., W. Nijdam, J. Sekulic, S. R. Chowdhury, C. J. M. van Rijn, A. van den Berg, J. E. ten Elshof, and D. H. A. Blank, 2005, Anal. Chem. 77, 178.

Schoch, R. B., 2006, Ph.D. thesis (EPFL).

Schoch, R. B., A. Bertsch, and P. Renaud, 2006, Nano Lett. 6, 543.

Schoch, R. B., L. F. Cheow, and J. Han, 2007, Nano Lett. 7, 3895 .

Schoch, R. B., and P. Renaud, 2005, Appl. Phys. Lett. 86, 253111.

Schoch, R. B., H. van Lintel, and P. Renaud, 2005, Phys. Fluids 17, 100604.

Scholze, H., 1988, J. Non-Cryst. Solids 102, 1.

Schwarz, B., and M. Schonhoff, 2002, Langmuir 18, 2964.

Semenov, A., T. Duke, and J.-L. Viovy, 1995, Phys. Rev. E 51, 1520.

Seth, L., M. Ashlie, Q. S. Randall, and W. Qian, 2007, Phys. Rev. Lett. 98, 226001.

Sheehan, P. E., and L. J. Whitman, 2005, Nano Lett. 5, 803.

Shim, J. H., J. Kim, G. S. Cha, H. Nam, R. J. White, H. S. White, and R. B. Brown, 2007, Anal. Chem. 79, 3568.

Siwy, Z., P. Apel, D. Baur, D. D. Dobrev, Y. E. Korchev, R. Neumann, R. Spohr, C. Trautmann, and K.-O. Voss, 2003, Surf. Sci. 532, 1061.

Siwy, Z., P. Apel, D. Dobrev, R. Neumann, R. Spohr, C. Trautmann, and K. Voss, 2003, Nucl. Instrum. Methods Phys. Res. B 208, 143.
Siwy, Z., and A. Fulinski, 2002a, Phys. Rev. Lett. 89, 198103. Siwy, Z., and A. Fulinski, 2002b, Phys. Rev. Lett. 89, 158101. Siwy, Z., and A. Fulinski, 2004, Am. J. Phys. 72, 567.

Siwy, Z., Y. Gu, H. A. Spohr, D. Baur, A. Wolf-Reber, R. Spohr, P. Apel, and Y. E. Korchev, 2002, Europhys. Lett. 60, 349.

Siwy, Z., E. Heins, C. C. Harrell, P. Kohli, and C. R. Martin, 2004, J. Am. Chem. Soc. 126, 10850.

Siwy, Z., I. D. Kosinska, A. Fulinski, and C. R. Martin, 2005, Phys. Rev. Lett. 94, 048102.

Siwy, Z., L. Trofin, P. Kohli, L. A. Baker, C. Trautmann, and C. R. Martin, 2005, J. Am. Chem. Soc. 127, 5000.

Siwy, Z. S., M. R. Powell, E. Kalman, R. D. Astumian, and R. S. Eisenberg, 2006, Nano Lett. 6, 473.

Slater, G., S. Guillouzic, M. Gauthier, J.-F. Mercier, M. Kenward, L. McCormick, and F. Tessier, 2002, Electrophoresis 23, 3791.

Slater, G., M. Kenward, L. McCormick, and M. Gauthier, 2003, Curr. Opin. Biotechnol. 14, 58.

Slonkina, E., and A. B. Kolomeisky, 2003, J. Chem. Phys. 118, 7112.

Smeets, R. M. M., U. F. Keyser, D. Krapf, M.-Y. Wu, N. H. Dekker, and C. Dekker, 2006, Nano Lett. 6, 89.

Smeets, R. M. M., U. F. Keyser, M. Y. Wu, N. H. Dekker, and C. Dekker, 2006, Phys. Rev. Lett. 97, 088101.

Smisek, D. L., and D. A. Hoagland, 1990, Science 248, 1221.

Smit, J. A. M., 1989, J. Colloid Interface Sci. 132, 413.

Smith, F. G., III, and W. Deen, 1983, J. Colloid Interface Sci. 91, 571.

Smith, F. G., III, and W. M. Deen, 1980, J. Colloid Interface Sci. 78, 444.

Smoluchowski, M. V., 1916, Z. Phys. Chem., Stoechiom. Verwandtschaftsl. 92, 129.

Smoluchowski, M. V., 1918, in Handbuch der Elektrizitaet und des Magnetismus, edited by L. Graetz (Johann Ambrosius Barth, Leipzig), Vol. 2.

Song, S., A. K. Singh, and B. J. Kirby, 2004, Anal. Chem. 76, 4589.

Sonnefeld, J., A. Gobel, and W. Vogelsberger, 1995, Colloid Polym. Sci. 273, 926.

Squires, T., and S. Quake, 2005, Rev. Mod. Phys. 77, 977.

Stahlberg, J., and B. Jonsson, 1996, Anal. Chem. 68, 1536.

Star, A., E. Tu, J. Niemann, J. C. P. Gabriel, C. S. Joiner, and C. Valcke, 2006, Proc. Natl. Acad. Sci. U.S.A. 103, 921.

Stein, D., M. Kruithof, and C. Dekker, 2004, Phys. Rev. Lett. 93, 035901.

Stein, D., F. H. J. van der Heyden, W. J. A. Koopmans, and C. Dekker, 2006, Proc. Natl. Acad. Sci. U.S.A. 103, 15853.

Stellwagen, N. C., C. Gelfi, and P. G. Righetti, 1997, Biopolymers 42, 687.

Stern, E., J. F. Klemic, D. A. Routenberg, P. N. Wyrembak, D. B. Turner-Evans, A. D. Hamilton, D. A. LaVan, T. M. Fahmy, and M. A. Reed, 2007, Nature 445, 519.

Stern, E., R. Wagner, F. J. Sigworth, R. Breaker, T. M. Fahmy, and M. A. Reed, 2007, Nano Lett. 7, 3405.

Storm, A., J. Chen, H. Zandbergen, and C. Dekker, 2005, Phys. Rev. E 71, 051903.

Storm, A. J., J. H. Chen, X. S. Ling, H. W. Zandbergen, and C. Dekker, 2003, Nat. Mater. 2, 537.

Storm, A. J., C. Storm, J. Chen, J.-F. Joanny, and C. Dekker, 2005, Nano Lett. 5, 1193.

Strathmann, H., 2004, Ion-Exchange Membrane Separation 
Processes, Vol. 9 of Membrane Science and Technology (Elsevier, Amsterdam).

Striemer, C. C., T. R. Gaborski, J. L. McGrath, and P. M. Fauchet, 2007, Nature 445, 749.

Sui, Z., and J. B. Schlenoff, 2003, Langmuir 19, 7829.

Sui, Z., and J. B. Schlenoff, 2004, Langmuir 20, 6026.

Sun, L., and R. Crooks, 2000, J. Am. Chem. Soc. 122, 12340.

Szymczyk, A., P. Fievet, B. Aoubiza, C. Simon, and J. Pagetti, 1999, J. Membr. Sci. 161, 275.

Tallarek, U., F. Leinweber, and I. Nischang, 2005, Electrophoresis 26, 391.

Tang, X. W., S. Bansaruntip, N. Nakayama, E. Yenilmez, Y. L. Chang, and Q. Wang, 2006, Nano Lett. 6, 1632.

Tans, S. J., A. R. M. Verschueren, and C. Dekker, 1998, Nature 393, 49.

Tas, N., J. Berenschot, P. Mela, H. Jansen, M. Elwenspoek, and A. van den Berg, 2002, Nano Lett. 2, 1031.

Tas, N., P. Mela, T. Kramer, J. Berenschot, and A. van den Berg, 2003, Nano Lett. 3, 1537.

Tas, N. R., J. Haneveld, H. V. Jansen, M. Elwenspoek, and A. van den Berg, 2004, Appl. Phys. Lett. 85, 3274.

Taylor, G., 1953, Proc. R. Soc. London, Ser. A 219, 186.

Tegenfeldt, J. O., O. Bakajin, C. F. Chou, S. S. Chan, R. Austin, W. Fann, L. Liou, E. Chan, T. Duke, and E. C. Cox, 2001, Phys. Rev. Lett. 86, 1378.

Tegenfeldt, J. O., C. Prinz, H. Cao, S. Y. Chou, W. W. Reisner, R. Riehn, Y. M. Wang, E. C. Cox, J. C. Sturm, P. Silberzan, and R. H. Austin, 2004, Proc. Natl. Acad. Sci. U.S.A. 101, 10979.

Tegenfeldt, J. O., C. Prinz, H. Cao, R. L. Huang, R. H. Austin, S. Y. Chou, E. C. Cox, and J. C. Sturm, 2004, Anal. Bioanal. Chem. 378, 1678.

Terrettaz, S., M. Mayer, and H. Vogel, 2003, Langmuir 19, 5567.

Tessier, F., J. Labrie, and G. W. Slater, 2002, Macromolecules 35, 4791.

Tessier, F., and G. Slater, 2002, Appl. Phys. A: Mater. Sci. Process. 75, 285.

Tessier, F., and G. W. Slater, 2006, Electrophoresis 27, 686.

Thelander, C., T. Martensson, M. T. Bjork, B. J. Ohlsson, M. W. Larsson, L. R. Wallenberg, and L. Samuelson, 2003, Appl. Phys. Lett. 83, 2052.

Tu, J., T. Huen, R. Szema, and M. Ferrari, 1999, J. Biomed. Microdev. 1, 113.

Turmel, C., E. Brassard, G. Slater, and J. Noolandi, 1990, Nucleic Acids Res. 18, 569.

Turner, S. W., M. Cabodi, and H. G. Craighead, 2002, Phys. Rev. Lett. 88, 128103.

Turner, S. W., A. M. Perez, A. Lopez, and H. G. Craighead, 1998, J. Vac. Sci. Technol. B 16, 3835.

Urban, F., H. L. White, and E. A. Strassner, 1934, J. Phys. Chem. 39, 311.

Vainshtein, P., and C. Gutfinger, 2002, J. Micromech. Microeng. 12, 252.

Vamvakaki, V., K. Tsagaraki, and N. Chaniotakis, 2006, Anal. Chem. 78, 5538.

van der Heyden, F. H. J., D. J. Bonthuis, D. Stein, C. Meyer, and C. Dekker, 2006, Nano Lett. 6, 2232.

van der Heyden, F. H. J., D. J. Bonthuis, D. Stein, C. Meyer, and C. Dekker, 2007, Nano Lett. 4, 1022.

van der Heyden, F. H. J., D. Stein, and C. Dekker, 2005, Phys. Rev. Lett. 95, 116104.

van der Horst, H. C., J. M. K. Timmer, T. Robbertsen, and J.
Leenders, 1995, J. Membr. Sci. 104, 205.

van Oudenaarden, A., and S. G. Boxer, 1999, Science 285, 1046.

Vercoutere, W., and M. Akeson, 2002, Curr. Opin. Chem. Biol. 6, 816.

Vercoutere, W., S. Winters-Hilt, H. Olsen, D. Deamer, D. Haussler, and M. Akeson, 2001, Nat. Biotechnol. 19, 248.

Viovy, J.-L., 2000, Rev. Mod. Phys. 72, 813.

Vlassiouk, I., and Z. S. Siwy, 2007, Nano Lett. 7, 552.

Volkmuth, W. D., and R. H. Austin, 1992, Nature 358, 600.

Vorrey, S., and D. Teeters, 2003, Electrochim. Acta 48, 2137.

Wang, D., and H. Dai, 2006, Appl. Phys. A: Mater. Sci. Process. 85, 217.

Wang, H., J. E. Dunning, A. P. H. Huang, J. A. Nyamwanda, and D. Branton, 2004, Proc. Natl. Acad. Sci. U.S.A. 101, 13472.

Wang, H. Y., and D. Branton, 2001, Nat. Biotechnol. 19, 622. Wang, H. Y., R. S. Foote, S. C. Jacobson, J. H. Schneibel, and J. M. Ramsey, 1997, Sens. Actuators B 45, 199.

Wang, J., 2005, Electroanalysis 17, 7.

Wang, M., N. Jing, I. H. Chou, G. L. Cote, and J. Kameoka, 2007, Lab Chip 7, 630.

Wang, W. U., C. Chen, K. H. Lin, Y. Fang, and C. M. Lieber, 2005, Proc. Natl. Acad. Sci. U.S.A. 102, 3208.

Wang, X.-L., T. Tsuru, S.-I. Nakao, and S. Kimura, 1995, J. Membr. Sci. 103, 117.

Wang, Y.-C., A. L. Stevens, and J. Han, 2005, Anal. Chem. 77, 4293.

Wang, Y. M., J. O. Tegenfeldt, W. Reisner, R. Riehn, X.-J. Guan, L. Guo, I. Golding, E. C. Cox, J. Sturm, and R. H. Austin, 2005, Proc. Natl. Acad. Sci. U.S.A. 102, 9796.

Wanunu, M., and A. Meller, 2007, Nano Lett. 7, 1580.

Washburn, E. W., 1921, Phys. Rev. 17, 273.

Wei, C., A. J. Bard, and S. W. Feldberg, 1997, Anal. Chem. 69, 4627.

Whitby, M., and N. Quirke, 2007, Nat. Nanotechnol. 2, 87.

Whitesides, G. M., 2006, Nature 442, 368.

Wikby, A., 1974, Electrochim. Acta 19, 329.

Wong, P. K., C. Y. Chen, T. H. Wang, and C. M. Ho, 2004, Anal. Chem. 76, 6908.

Wu, R., H. Zou, M. Ye, Z. Lei, and J. Ni, 2001, Electrophoresis 22, 544 .

Wu, Y., J. Xiang, C. Yang, W. Lu, and C. M. Lieber, 2004, Nature 430, 61.

Xia, Y. N., P. D. Yang, Y. G. Sun, Y. Y. Wu, B. Mayers, B. Gates, Y. D. Yin, F. Kim, and Y. Q. Yan, 2003, Adv. Mater. (Weinheim, Ger.) 15, 353.

Xiang, J., W. Lu, Y. J. Hu, Y. Wu, H. Yan, and C. M. Lieber, 2006, Nature 441, 489.

Xiangchun Xuan, D. L., 2007, Electrophoresis 28, 627.

Xuan, X., 2007, Anal. Chem. 79, 7928.

Yang, C., and D. Li, 1997, J. Colloid Interface Sci. 194, 95.

Yang, K.-L., and S. Yiacoumi, 2002, J. Chem. Phys. 117, 8499.

Yaroshchuk, A., O. Zhukova, M. Ulbricht, and V. Ribitsch, 2005, Langmuir 21, 6872.

Yaroshchuk, A. E., 2001a, J. Colloid Interface Sci. 238, 381.

Yaroshchuk, A. E., 2001b, Sep. Purif. Technol. 22-23, 143.

Yates, D. E., S. Levine, and T. W. Healy, 1974, J. Chem. Soc., Faraday Trans. 1 70, 1807.

Yi, M., K.-H. Jeong, and L. P. Lee, 2005, Biosens. Bioelectron. 20, 1320.

Yu, G., A. Cao, and C. M. Lieber, 2007, Nat. Nanotechnol. 2, 372. 
Zeng, Y., and D. J. Harrison, 2007, Anal. Chem. 79, 2289.

Zeze, D. A., R. D. Forrest, J. D. Carey, D. C. Cox, I. D. Robertson, B. L. Weiss, and S. R. P. Silva, 2002, J. Appl. Phys. 92, 3624.

Zhang, W. M., Y. G. Wang, J. Li, J. M. Xue, H. Ji, Q. Ouyang, J. Xu, and Y. Zhang, 2007, Appl. Phys. Lett. 90, 163102.

Zhang, X. G., P. S. Krstic, R. Zikic, J. C. Wells, and M. Fuentes-Cabrera, 2006, Biophys. J. 91, L4.

Zhao, Q., G. Sigalov, V. Dimitrov, B. Dorvel, U. Mirsaidov, S.

Sligar, A. Aksimentiev, and G. Timp, 2007, Nano Lett. 7,
1680.

Zheng, G., F. Patolsky, Y. Cui, W. U. Wang, and C. Lieber, 2005, Nat. Biotechnol. 23, 1294.

Zheng, G. F., W. Lu, S. Jin, and C. M. Lieber, 2004, Adv. Mater. (Weinheim, Ger.) 16, 1890.

Zhong, Z. H., Y. Fang, W. Lu, and C. M. Lieber, 2005, Nano Lett. 5, 1143.

Zhou, X., J. M. Moran-Mirabal, H. G. Craighead, and P. L. McEuen, 2007, Nat. Nanotechnol. 2, 185.

Zydney, A., and N. Pujar, 1998, Colloids Surf., A 138, 133. 